\title{
Birds of Estação Ecológica da Serra das Araras, state of Mato Grosso, Brazil: additions and review
}

\author{
Breno Dias Vitorino, Angélica Vilas Boas da Frota, Solange Kimie Ikeda Castrillon, \\ Josué Ribeiro da Silva Nunes
}

Programa de Pós-graduação em Ciências Ambientais, Universidade do Estado de Mato Grosso, Centro de Pesquisa em Limnologia, Biodiversidade e Etnobiologia no Pantanal, Av. Santos Dumont, s/nº - Cidade Universitária (Bloco II), Cáceres, Mato Grosso, 78200-000, Brazil.

Corresponding author: Breno Dias Vitorino, brenovitorino@gmail.com

\begin{abstract}
We present an inventory, followed by a consolidated list, for the avifauna of Estação Ecológica da Serra das Araras (EESA), state of Mato Grosso, Brazil. In our expeditions, we identified 287 species, $82 \%$ of them documented. Of these, 28 species are new records for EESA. Six are threatened, such as Lophornis gouldii (Lesson, 1832). Other noteworthy records include the first documentation of Oxyruncus cristatus Swainson, 1821, for Mato Grosso. The consolidated list includes 458 species, of which 255 were assigned to the primary list and 203 to the secondary list. The significant number of species compiled herein places EESA among the most species-rich protected areas of Brazil, especially in regard to areas not predominantly covered by forest. In terms of species composition, this is a community typical of an ecotone environment, featuring many species characteristic of the Cerrado and the Amazon and some of the Pantanal wetland.
\end{abstract}

\section{Key words}

Neotropical birds; ornithological survey; biogeography; biodiversity; ecotone; protected area; Província Serrana.

Academic editor: Galo Buitrón-Jurado | Received 14 April 2018 | Accepted 25 August 2018 | Published 26 October 2018

Citation: Vitorino BD, Frota AVB, Castrillon SKI, Nunes JRS (2018) Birds of Estação Ecológica da Serra das Araras, state of Mato Grosso, Brazil: additions and review. Check List 14 (5): 893-922. https://doi.org/10.15560/14.5.893

\section{Introduction}

The Estação Ecológica da Serra das Araras (EESA) is a protected area, created by decree 87.222 of 1982 , in southwestern Mato Grosso state, Brazil. Due to its location in an area bordering Bolivia, it is highlighted in the international context (Brazil 2016a). The main objective of the EESA is to preserve nature in accordance with Federal Law 9.985 of 2000, which establishes criteria and norms for the creation, implementation and management of protected areas in Brazil. Among the benefits of its creation according to Hassler (2005), can be highlighted as follows: biodiversity and ecological processes conservation, maintenance of water resources, improvement in air quality, water and soil.

With regard to avifauna, EESA has had 5 studies so far. Silva and Oniki (1988), Willis and Oniki (1990), 


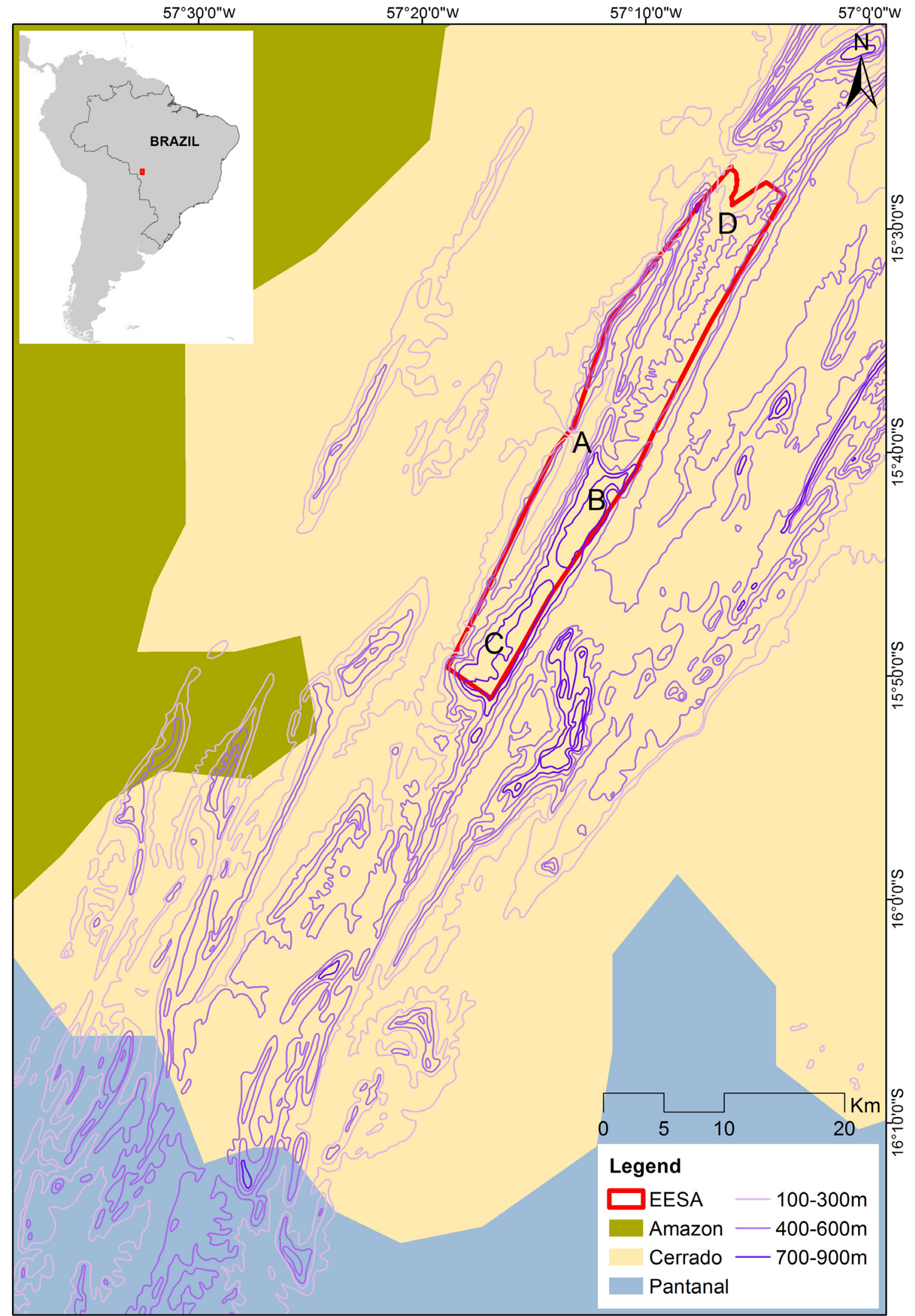

Figure 1. Location of Estação Ecológica da Serra das Araras (EESA), state of Mato Grosso, Brazil, with isolines representing the Província Serrana elevational gradient.

Oniki and Willis (1999) and Oniki and Oliveira (2002) present data collected in expeditions undertaken from 1986 to 1988, while Valadão (2012) presents data collected in 2006 and 2007 that also make up the EESA management plan (Brazil 2016a). These works provide relevant information on the occurrence of several species in the 
region, including many noteworthy records, especially of Columbina cyanopis (Pelzeln, 1870), one of the rarest birds in the world (Silva and Oniki 1988, Valadão 2012).

Although the avifauna of EESA is relatively well known, the making of a new inventory 10 years after the last one allows an historical evaluation of the bird community and adds relevant information to the ongoing understanding of ecological patterns and distribution of the species involved. We aim to present an up-to-date inventory of the bird species that occur in EESA, followed by a compilation and review of the bird records for the EESA, as until now this protected area lacked a consolidated list based on standardized criteria.

\section{Methods}

Study area. The EESA is a 28,700 ha protected area mostly situated in Porto Estrela Municipality and, to a lesser degree, in Cáceres Municipality, state of Mato Grosso, Brazil. It is located in the geomorphological unit of Província Serrana, a corridor of parallel mountain in the Cerrado in transition with the Pantanal wetland and Amazon (Brazil 2016a). The climate is considered Aw in the Köppen classification, with annual rainfall around $1500 \mathrm{~mm}$ and maximum and minimum average temperatures around $32{ }^{\circ} \mathrm{C}$ and $20^{\circ} \mathrm{C}$, respectively (Kottek et al. 2006). EESA presents high environmental heterogeneity, including savanna, forest and grassland formations (Brazil 2016a). To cover a greater number of EESA habitats, we concentrated the bird survey in 4 sampling areas, A, B, C and D (Fig. 1).

Area A: central region of the Salobra river valley, where the EESA headquarters are located $\left(15^{\circ} 39^{\prime} 10.96^{\prime \prime} \mathrm{S}\right.$, $057^{\circ} 12^{\prime} 52.54^{\prime \prime}$ W) (Fig. 2A). It consists of a vegetation mosaic comprising semideciduous seasonal forest with areas dominated by Attalea speciosa Mart. Ex Spreng, the savanna-like cerrado sensu stricto (Fig. 2b), riparian forest and a small human-altered area surrounding the EESA headquarters. The area is under the influence of the Camarinha and Salobinha rivers. The elevation is around $225 \mathrm{~m}$, with no great variation.

Area B: region situated on the upper eastern part that forms the Salobra River valley and includes Cabeceira

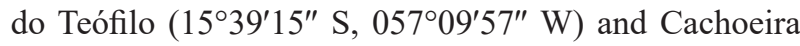
da Andorinha $\left(15^{\circ} 41^{\prime} 05.05^{\prime \prime} \mathrm{S}, 057^{\circ} 12^{\prime} 30^{\prime \prime} \mathrm{W}\right)$. Predominant vegetation is cerrado sensu stricto (Fig. 2C); open cerrado, small areas of cerrado rupestre (a type of cerrado that grows on rocky terrain), riparian forest and lower montane forest formations are also present. The elevation ranges from 500 to $650 \mathrm{~m}$, with the higher parts located on the eastern part of EESA. Around Cachoeira da Andorinha lies the EESA's largest area of campo limpo (open grassland) (Fig. 2D). The region also harbors areas of cerrado rupestre, campo sujo (grassland with shrubs and scattered small trees) and open cerrado. The elevation ranges from 750 to $860 \mathrm{~m}$.

Area C: southern part, known as Jauquara region, due to the nearby river of the same name. The environ- ments sampled encompass formations with rock outcrops $\left(15^{\circ} 46^{\prime} 30^{\prime \prime} \mathrm{S}, 057^{\circ} 15^{\prime} 16^{\prime \prime} \mathrm{W}\right)$ (Fig. 2E), farmland located at the EESA's southernmost region $\left(15^{\circ} 49^{\prime} 40^{\prime \prime} \mathrm{S}, 057^{\circ} 17^{\prime} 20^{\prime \prime}\right.$ $\mathrm{W})$, cerrado sensu stricto, campo sujo and lower montane forest. The elevation ranges from around 700 to $850 \mathrm{~m}$.

Area D: northern part, strongly influenced by the Três Ribeirões River $\left(15^{\circ} 28^{\prime} 56^{\prime \prime} \mathrm{S}, 057^{\circ} 05^{\prime} 42^{\prime \prime} \mathrm{W}\right)$. Predominant vegetation formations are deciduous seasonal forest (Fig. 2F) and semideciduous seasonal forest (Fig. 2G) with areas dominated by $A$. speciosa (Fig. $2 \mathrm{H}$ ), areas of cerrado sensu stricto and riparian forest. The elevation ranges from 250 to $500 \mathrm{~m}$.

Data collection and analysis. Additions. We call additions the contributions resulting from our field sampling (in situ). All fieldwork was conducted under the proper permits: Sistema de Autorização e Informação em Biodiversidade/Instituto Brasileiro do Meio Ambiente e dos Recursos Naturais Renováveis (54984-1), Centro Nacional de Pesquisa e Conservação de Aves Silvestres (4156/1) and Comitê de Ética em Pesquisas com Animais da Universidade do Estado de Mato Grosso (009/2016).

We collected data from October 2016 to September 2017. We identified bird species by sight and sound, using $10 \times 40$ binoculars, photo cameras, a digital audio recorder and specialized literature such as Sick (1997) and del Hoyo et al. (2017). Whenever possible, we documented bird species with photographs and audio recordings, which were archived on Xeno-canto (https://www.xenocanto.org), Wikiaves (http://www.wikiaves.com.br) and Macaulay Library (https://www.macaulaylibrary.org). We also collected some specimens and deposited them in the ornithological collection of Universidade Federal de Mato Grosso (UFMT).

For the survey, we used 3 complementary methods: transect (Bibby et al. 2000), capture with mist nets (Karr 1981, Keyes and Grue 1982, Roos 2010) and MacKinnon lists (MacKinnon 1991, Herzog et al. 2002).

Transect: in Area A, we set up a $3.5 \mathrm{~km}$ trail running across cerrado sensu stricto, riparian forest and semideciduous seasonal forest habitats. This trail was sampled 38 times throughout the year, 19 in the dry season and 19 in the wet season. Each sampling lasted on average 3 hours, amounting to a total effort of about 110 hours. We considered as records all the specimens detected at a distance of up to about $50 \mathrm{~m}$ from the observer.

Capture with mist nets: we defined 4 plots in Area A (P1, P2, P3 and P4) covering different phytophysiognomies (Table 1). Each plot was sampled for 2 consecutive days per month over a period of 1 year. We used 7 mist nets $(12 \times 3 \mathrm{~m} ; 20 \mathrm{~mm}$ mesh $)$ in each plot for 5 hours per day, totaling 30,24 $\mathrm{m}^{2} \mathrm{~h}$ (Straube and Bianconi 2002). Capture procedure followed Brazil (1994).

MacKinnon lists: in Areas B, C and D we surveyed the birds by making MacKinnon lists with 10 species, walking along pre-existing trails in the morning, afternoon and occasionally at night. Total number of lists obtained for each area was 32, 36 and 42, respectively. 

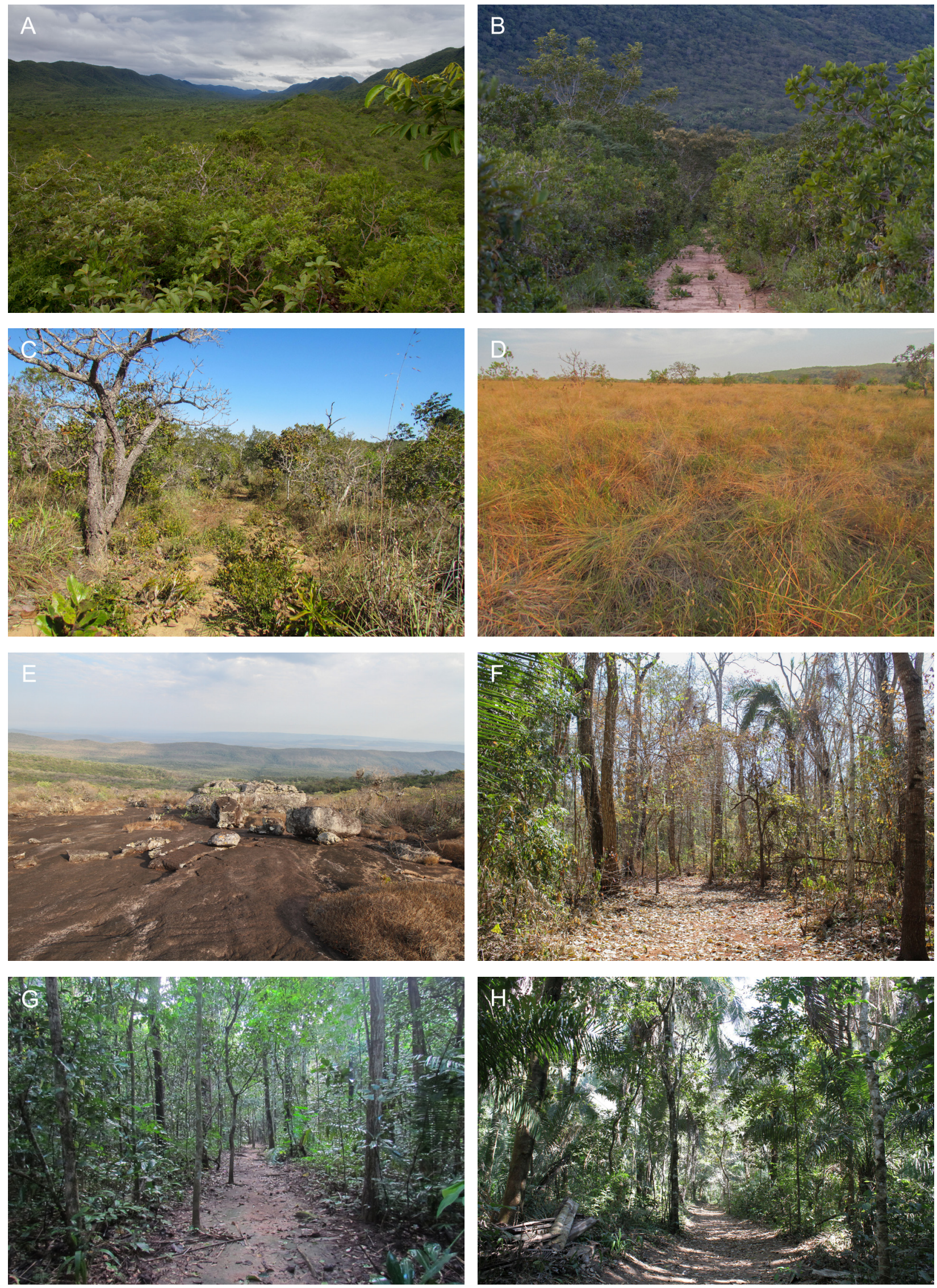

Figure 2. A. Central region of the Salobra River valley (Area A). B. Cerrado sensu stricto (Area A). C. Cerrado sensu stricto (Area B). D. Campo Limpo (Area B). E. Rocky formations (Area C). F. Deciduous seasonal forest (Area D). G. Semideciduous seasonal forest (Area A). H. Seasonal forest dominated by Attalea speciosa Mart. ex Spreng (Area D). (Photos: BDV). 
We defined frequency of species occurrence (FO) as the number of samples where a given species was recorded divided by the total number of samples, according to D'Angelo Neto et al. (1998). We also included randomly recorded species when putting together the species list.

Birds of Estação Ecológica da Serra das Araras: review. This review and compilation work aims to generate a standardized list and increase the credibility of the EESA species list, according to the guidelines laid down by the Brazilian Ornithological Records Committee (Piacentini et al. 2015). We combined the results of our inventory for EESA with occurrence records from the literature (Silva and Oniki 1988, Willis and Oniki 1990, Oniki and Oliveira 2002, Valadão 2012) and 2 species mentioned exclusively in the management plan (Brazil 2016a). We did not include the species cited in Oniki and Willis (1999), as record localities were imprecise. We also searched for documented records in scientific collections (UFMT, Museu Paraense Emílio Goeldi, Museu de Zoologia da Universidade de São Paulo, Universidade Católica de Minas Gerais, Universidade Federal de Minas Gerais, Universidade Federal do Rio de Janeiro and Instituto Nacional de Pesquisas da Amazônia) and in the databases Wikiaves, Xeno-canto and Macaulay Library (accessed in March 2018). Each species listed was then assessed and assigned to 1 of 3 different lists:

Primary list: includes species with occurrence records in EESA with at least one documented evidence item, in the form of either a complete or a partial specimen, a photograph or a sound recording. Documented evidence must provide a trustworthy diagnosis of the taxon in question and be available for reassessment.

Secondary list: includes species with occurrence records at EESA for which documented evidence is unknown or unavailable. These species were considered of "probable occurrence" at the EESA, based on currently known distribution and dispersal patterns.

Tertiary list: includes species with occurrence records at EESA for which evidence is questionable, invalid or non-existent, and whose occurrence at the EESA seems improbable in the light of current knowledge. These species were excluded from the consolidated list.

The consolidated list is thus composed of species from the primary and secondary lists. Presentation of the evidence of records listed for EESA was based on Carlos et al. (2010) and used: "Indication of Occurrence" for species recorded in the field but not documented, either in our field inventory or in inventories available in the literature; "A-level documentation" for species with specimens deposited in scientific collections; "B-level documentation" for species documented with photographs or audio recordings. Species conservation status follows the executive summary of the Brazil Red Book of Threatened Species of Fauna (Brazil 2016b) at the national level and the Red List of Threatened Species (IUCN 2017) at the global level. For Cerrado endemics, we followed Silva (1995), Cavalcanti (1999), Sick (1997) and Silva and Bates (2002). For taxonomic classification, we followed Piacentini et al. (2015).

\section{Results}

Additions. During the in situ sampling, we recorded a total of 287 bird species, 270 through systematic sampling and 17 through random observations. Out of them, $236(82 \%$ of the total) are documented in this article with vouchers. Systematically, richness for area A equaled 195 species, with 186 recorded during the transect and 79 captured with mist nets. For areas B, C and D, 114, 128 and 111 species were recorded with the MacKinnon lists, respectively (Table 2 ).

Analyzing the data we obtained with the transect method, the species with the highest frequency of occurrence (FO) indices in relation to the transects were Basileuterus culicivorus (Deppe, 1830) (FO = 1.0), Formicivora grisea (Boddaert, 1783) $(\mathrm{FO}=0.97)$, Thamnophilus pelzelni Hellmayr, $1924(\mathrm{FO}=0.97)$, Neopelma pallescens (Lafresnaye, 1853) (FO =0.97), Pipra fasciicauda Hellmayr, $1906(\mathrm{FO}=0.97)$ and Myiothlypis flaveola Baird, 1865 (FO = 0.97). Analyzing the captures, the most frequent species was $P$. fasciicauda (FO $=1.00)$, followed by $N$. pallescens $(\mathrm{FO}=0.92)$. In terms of MacKinnon lists in area $\mathrm{B}$, the most frequent species were Euphonia chlorotica (Linnaeus, 1766) $(\mathrm{FO}=0.34)$ and Patagioenas speciosa (Gmelin, 1789) $(\mathrm{FO}=0.31)$; in area C, Myrmophylax atrothorax (Boddaert, 1783) $(\mathrm{FO}=$ 0.50) and Hypocnemis ochrogyna Zimmer, 1932 (FO = $0.46)$; in area D, Tangara sayaca (Linnaeus, 1766) $(\mathrm{FO}=$ $0.37)$, Eupsittula aurea (Gmelin, 1788) $(\mathrm{FO}=0.30)$ and Cyclarhis gujanensis (Gmelin, 1789) $(\mathrm{FO}=0.30)$.

Of the species we recorded, 28 are new records for EESA. We found 4 Near Threatened species and 6 Vulnerable species and recorded 6 species endemic to Cerrado. Below, we present accounts for these species, as well as some noteworthy records.

Table 1. Predominant phytophysiognomies of the plots sampled with mist nets at Estação Ecológica da Serra das Araras, state of Mato Grosso, Brazil and respective geographical coordinates.

\begin{tabular}{llll}
\hline Plot & Predominant phytophysiognomy & Latitude (S) & Longitude (W) \\
\hline P1 & Semideciduous seasonal forest & $15^{\circ} 39^{\prime} 0.94^{\prime \prime}$ & $057^{\circ} 12^{\prime} 42.73^{\prime \prime}$ \\
P2 & Cerrado sensu stricto & $15^{\circ} 39^{\prime} 20.06^{\prime \prime}$ & $057^{\circ} 12^{\prime} 57.65^{\prime \prime}$ \\
P3 & Gallery forest & $15^{\circ} 39^{\prime} 44.49^{\prime \prime}$ & $057^{\circ} 13^{\prime} 12.99^{\prime \prime}$ \\
P4 & Cerrado sensu stricto with gallery forest influence & $15^{\circ} 39^{\prime} 53.59^{\prime \prime}$ & $057^{\circ} 13^{\prime} 31.92^{\prime \prime}$ \\
\hline
\end{tabular}


Table 2. List of birds recorded at Estação Ecológica da Serra das Araras, state of Mato Grosso, Brazil from October 2016 to September 2017. $\mathrm{VU}^{*}=$ Vulnerable (Brazil 2016b); VU = Vulnerable (IUCN 2016); NT = Near Threatened; $C E=$ cerrado endemic; $(F O)=$ frequency of species occurrence for area ( $\mathrm{T}$, based on transect method and $\mathrm{M}$, based on capture with mist nets method). Species without FO information were recorded randomly. Catalog numbers in Voice and Photo columns are hyperlinked to the the online databases: Xeno-canto, WikiAves, or Macaulay Library.

\begin{tabular}{|c|c|c|c|c|c|c|c|c|c|}
\hline \multirow[b]{2}{*}{ Taxon name } & \multirow[b]{2}{*}{ English name } & \multicolumn{3}{|c|}{ Vouchers } & \multicolumn{5}{|c|}{ Area } \\
\hline & & Specimen & Voice & Photo & $\begin{array}{c}\text { A } \\
\text { (FO) } \\
\text { T }\end{array}$ & $\begin{array}{c}\text { A } \\
\text { (FO) } \\
\text { M }\end{array}$ & $\begin{array}{c}\text { B } \\
\text { (FO) }\end{array}$ & $\begin{array}{l}\mathrm{C} \\
\text { (FO) }\end{array}$ & $\begin{array}{c}\text { D } \\
\text { (FO) }\end{array}$ \\
\hline \multicolumn{10}{|l|}{ Rheiformes } \\
\hline \multicolumn{10}{|l|}{ Rheidae } \\
\hline Rhea americana (Linnaeus, 1758) NT & Greater Rhea & & & WA2737752 & & & & 0.03 & \\
\hline \multicolumn{10}{|l|}{ Tinamiformes Huxley, 1872} \\
\hline \multicolumn{10}{|l|}{ Tinamidae } \\
\hline Tinamus tao Temminck, 1815 VU, VU* & Gray Tinamou & & & & 0.37 & & 0.03 & 0.03 & 0.4 \\
\hline Crypturellus soui (Hermann, 1783) & Little Tinamou & & XC400315 & & 0.37 & & & 0.07 & \\
\hline Crypturellus undulatus (Temminck, 1815) & Undulated Tinamou & & XC387954 & & 0.58 & & 0.09 & 0.10 & 0.13 \\
\hline Crypturellus parvirostris (Wagler, 1827) & Small-billed Tinamou & & XC400253 & & & & 0.03 & 0.07 & \\
\hline Crypturellus tataupa (Temminck, 1815) & Tataupa Tinamou & & XC404460 & WA2760485 & 0.11 & & & & 0.4 \\
\hline Rhynchotus rufescens (Temminck, 1815) & Red-winged Tinamou & & XC400254 & WA2739639 & & & 0.03 & 0.27 & \\
\hline \multicolumn{10}{|l|}{ Galliformes } \\
\hline \multicolumn{10}{|l|}{ Cracidae } \\
\hline Penelope superciliaris Temminck, 1815 & Rusty-margined Guan & & & WA2890256 & 0.11 & & 0.03 & 0.10 & 0.02 \\
\hline Aburria cujubi (Pelzeln, 1858) & Red-throated Piping-Guan & & & WA2741413 & 0.03 & & & & \\
\hline Ortalis canicollis (Wagler, 1830) & Chaco Chachalaca & & & & 0.08 & & & & \\
\hline Crax fasciolata Spix, 1825 VU & Bare-faced Curassow & & XC402823 & WA2742645 & 0.24 & & & 0.07 & 0.4 \\
\hline \multicolumn{10}{|l|}{ Odontophoridae } \\
\hline Odontophorus gujanensis (Gmelin, 1789) NT & Marbled Wood-Quail & & XC400324 & & 0.26 & & & 0.07 & 0.02 \\
\hline \multicolumn{10}{|l|}{ Podicipediformes } \\
\hline Podicipedidae & & & & & & & & & \\
\hline Tachybaptus dominicus (Linnaeus, 1766) & Least Grebe & & & WA2732381 & & & & 0.07 & \\
\hline Ciconiiformes & & & & & & & & & \\
\hline Ciconiidae & & & & & & & & & \\
\hline Jabiru mycteria (Lichtenstein, 1819) & Jabiru & & & & & & & & \\
\hline Mycteria americana Linnaeus, 1758 & Wood Stork & & & WA2737798 & & & 0.06 & 0.03 & \\
\hline Pelecaniformes & & & & & & & & & \\
\hline Ardeidae & & & & & & & & & \\
\hline Tigrisoma lineatum (Boddaert, 1783) & Rufescent Tiger-Heron & & & & & & & & \\
\hline Agamia agami (Gmelin, 1789) VU & Agami Heron & & & & & & & & \\
\hline Bubulcus ibis (Linnaeus, 1758) & Cattle Egret & & & & & & & & \\
\hline Threskiornithidae & & & & & & & & & \\
\hline Mesembrinibis cayennensis (Gmelin, 1789) & Green Ibis & & & & 0.05 & & & & \\
\hline Theristicus caudatus (Boddaert, 1783) & Buff-necked lbis & & & WA2742651 & & & & 0.03 & \\
\hline Cathartiformes & & & & & & & & & \\
\hline Cathartidae & & & & & & & & & \\
\hline Cathartes aura (Linnaeus, 1758) & Turkey Vulture & & & WA2722294 & 0.03 & & 0.09 & & 0.4 \\
\hline Cathartes burrovianus Cassin, 1845 & $\begin{array}{l}\text { Lesser Yellow-headed } \\
\text { Vulture }\end{array}$ & & & & & & & & \\
\hline Cathartes melambrotus Wetmore, 1964 & $\begin{array}{l}\text { Greater Yellow-headed } \\
\text { Vulture }\end{array}$ & & & & & & & & \\
\hline Coragyps atratus (Bechstein, 1793) & Black Vulture & & & WA2731330 & 0.05 & & 0.13 & 0.07 & 0.02 \\
\hline Sarcoramphus papa (Linnaeus, 1758) & King Vulture & & & WA2470868 & 0.08 & & 0.13 & & 0.4 \\
\hline Accipitriformes & & & & & & & & & \\
\hline Accipitridae & & & & & & & & & \\
\hline Leptodon cayanensis (Latham, 1790) & Gray-headed Kite & & & & & & & & 0.02 \\
\hline Chondrohierax uncinatus (Temminck, 1822) & Hook-billed Kite & & & WA2403490 & 0.03 & & & & \\
\hline Harpagus bidentatus (Latham, 1790) & Double-toothed Kite & & & & & & & & \\
\hline Harpagus diodon (Temminck, 1823) & Rufous-thighed Kite & & & WA2858435 & 0.03 & & & & \\
\hline Accipiter superciliosus (Linnaeus, 1766) & Tiny Hawk & & & & 0.05 & & & & \\
\hline Accipiter bicolor (Vieillot, 1817) & Bicolored Hawk & & & & 0.03 & & & & \\
\hline Ictinia plumbea (Gmelin, 1788) & Plumbeous Kite & & & WA2731340 & & & 0.06 & 0.03 & \\
\hline Urubitinga urubitinga (Gmelin, 1788) & Great Black Hawk & & & WA2731342 & & & & & 0.02 \\
\hline Rupornis magnirostris (Gmelin, 1788) & Roadside Hawk & & XC404561 & WA2733685 & 0.32 & & 0.03 & 0.17 & \\
\hline Geranoaetus albicaudatus (Vieillot, 1816) & White-tailed Hawk & & & WA2722298 & 0.03 & & 0.03 & 0.03 & \\
\hline Pseudastur albicollis (Latham, 1790) & White Hawk & & & WA2884986 & 0.03 & & & & \\
\hline Buteo nitidus (Latham, 1790) & Gray-lined Hawk & & & WA2732377 & & & & 0.03 & 0.02 \\
\hline Spizaetus tyrannus (Wied, 1820) & Black Hawk-Eagle & & & WA2885005 & & & & & \\
\hline Spizaetus ornatus (Daudin, 1800) NT & Ornate Hawk-Eagle & & XC400234 & & 0.08 & & 0.03 & & \\
\hline Eurypygiformes & & & & & & & & & \\
\hline Eurypygidae & & & & & & & & & \\
\hline Eurypyga helias (Pallas, 1781) & Sunbittern & & XC400205 & WA2739643 & 0.03 & & & & \\
\hline Gruiformes & & & & & & & & & \\
\hline Rallidae & & & & & & & & & \\
\hline Aramides cajaneus (Statius Müller, 1776) & Gray-necked Wood-Rail & & & & 0.03 & & & & 0.02 \\
\hline
\end{tabular}




\begin{tabular}{|c|c|c|c|c|c|c|c|c|c|}
\hline \multirow[b]{2}{*}{ Taxon name } & \multirow[b]{2}{*}{ English name } & \multicolumn{3}{|c|}{ Vouchers } & \multicolumn{5}{|c|}{ Area } \\
\hline & & Specimen & Voice & Photo & $\begin{array}{c}A \\
\text { (FO) } \\
T\end{array}$ & $\begin{array}{c}\text { A } \\
\text { (FO) } \\
M\end{array}$ & $\begin{array}{c}\text { B } \\
\text { (FO) }\end{array}$ & $\begin{array}{c}\mathrm{C} \\
\text { (FO) }\end{array}$ & $\begin{array}{c}D \\
\text { (FO) }\end{array}$ \\
\hline Mustelirallus albicollis (Vieillot, 1819) & Ash-throated Crake & & XC400268 & & & & & 0.03 & \\
\hline \multicolumn{10}{|l|}{ Charadriiformes } \\
\hline \multicolumn{10}{|l|}{ Charadriidae } \\
\hline Vanellus chilensis (Molina, 1782) & Southern Lapwing & & & WA2742650 & 0.03 & & & 0.07 & \\
\hline \multicolumn{10}{|l|}{ Jacanidae } \\
\hline Jacana jacana (Linnaeus, 1766) & Wattled Jacana & & & WA2760489 & & & & 0.03 & \\
\hline \multicolumn{10}{|l|}{ Columbiformes } \\
\hline \multicolumn{10}{|l|}{ Columbidae } \\
\hline Columbina talpacoti (Temminck, 1810) & Ruddy Ground-Dove & & & WA2731329 & & & & 0.13 & 0.02 \\
\hline Columbina squammata (Lesson, 1831) & Scaled Dove & & & WA2731329 & & & 0.03 & & \\
\hline Claravis pretiosa (Ferrari-Perez, 1886) & Blue Ground-Dove & & XC400177 & WA2760487 & 0.24 & 0.08 & & & \\
\hline Patagioenas speciosa (Gmelin, 1789) & Scaled Pigeon & & & WA2742647 & 0.47 & & 0.31 & 0.17 & 0.13 \\
\hline Patagioenas picazuro (Temminck, 1813) & Picazuro Pigeon & & & & 0.03 & & & 0.07 & 0.02 \\
\hline Patagioenas cayennensis (Bonnaterre, 1792) & Pale-vented Pigeon & & & WA2732454 & 0.03 & & 0.03 & 0.13 & 0.02 \\
\hline Patagioenas plumbea (Vieillot, 1818) & Plumbeous Pigeon & & XC400175 & WA2850097 & 0.84 & & 0.09 & & 0.28 \\
\hline Leptotila verreauxi Bonaparte, 1855 & White-tipped Dove & & XC387962 & WA2741416 & 0.71 & 0.08 & 0.19 & 0.23 & 0.09 \\
\hline Leptotila rufaxilla (Richard \& Bernard, 1792) & Gray-fronted Dove & & XC404589 & WA2741417 & 0.34 & & 0.06 & & 0.09 \\
\hline Geotrygon montana (Linnaeus, 1758) & Ruddy Quail-Dove & & & WA2760483 & 0.11 & & & & \\
\hline Cuculiformes & & & & & & & & & \\
\hline Cuculidae & & & & & & & & & \\
\hline Piaya cayana (Linnaeus, 1766) & Squirrel Cuckoo & & & WA2866108 & 0.50 & & 0.16 & 0.03 & \\
\hline Coccyzus euleri Cabanis, 1873 & Pearly-breasted Cuckoo & & & & & & & & \\
\hline Crotophaga ani Linnaeus, 1758 & Smooth-billed Ani & & XC400252 & WA2857389 & & & & 0.07 & 0.02 \\
\hline Guira guira (Gmelin, 1788) & Guira Cuckoo & & & WA2739640 & & & & 0.07 & \\
\hline Dromococcyx pavoninus Pelzeln, 1870 & Pavonine Cuckoo & & XC400266 & & 0.05 & & & 0.03 & 0.02 \\
\hline Strigiformes & & & & & & & & & \\
\hline Strigidae & & & & & & & & & \\
\hline Megascops choliba (Vieillot, 1817) & Tropical Screech-Owl & & XC387966 & & & & 0.03 & & \\
\hline Megascops usta (Sclater, 1858) & Austral Screech-Owl & & XC400323 & & 0.08 & & & & \\
\hline Pulsatrix perspicillata (Latham, 1790) & Spectacled Owl & & XC400215 & WA2732382 & 0.08 & & & & 0.02 \\
\hline Strix virgata (Cassin, 1849) & Mottled Owl & & & & 0.03 & & & & \\
\hline Glaucidium brasilianum (Gmelin, 1788) & Ferruginous Pygmy-Owl & & XC404263 & & 0.24 & & 0.03 & 0.03 & \\
\hline Athene cunicularia (Molina, 1782) & Burrowing Owl & & & WA2732378 & & & & 0.03 & \\
\hline Asio clamator (Vieillot, 1808) & Striped Owl & & XC404556 & WA2741414 & & & 0.03 & & \\
\hline Nyctibiiformes & & & & & & & & & \\
\hline Nyctibiidae & & & & & & & & & \\
\hline Nyctibius griseus (Gmelin, 1789) & Common Potoo & & & WA2741362 & 0.08 & & 0.03 & 0.03 & \\
\hline Caprimulgiformes & & & & & & & & & \\
\hline Caprimulgidae & & & & & & & & & \\
\hline Antrostomus rufus (Boddaert, 1783) & Rufous Nightjar & & XC387948 & & 0.05 & & & 0.03 & \\
\hline Lurocalis semitorquatus (Gmelin, 1789) & Short-tailed Nighthawk & & XC395165 & & 0.03 & & & & \\
\hline Nyctidromus albicollis (Gmelin, 1789) & Common Pauraque & & XC387953 & & 0.29 & & 0.06 & 0.07 & 0.02 \\
\hline Hydropsalis parvula (Gould, 1837) & Little Nightjar & & XC402783 & & 0.08 & & & 0.07 & \\
\hline Hydropsalis longirostris (Bonaparte, 1825) & Band-winged Nightjar & & XC392826 & & & & 0.03 & & \\
\hline Hydropsalis torquata (Gmelin, 1789) & Scissor-tailed Nightjar & & XC387964 & & 0.11 & 0.08 & 0.03 & 0.03 & \\
\hline Apodiformes & & & & & & & & & \\
\hline Apodidae & & & & & & & & & \\
\hline Cypseloides senex (Temminck, 1826) & Great Dusky Swift & & & WA2890263 & 0.05 & & 0.03 & & \\
\hline Streptoprocne zonaris (Shaw, 1796) & White-collared Swift & & & WA2723259 & 0.03 & & & & \\
\hline Trochilidae & & & & & & & & & \\
\hline Glaucis hirsutus (Gmelin, 1788) & Rufous-breasted Hermit & & & & & 0.25 & & & \\
\hline Phaethornis nattereri Berlepsch, 1887 & Cinnamon-throated Hermit & & & & & & & & 0.07 \\
\hline Phaethornis ruber (Linnaeus, 1758) & Reddish Hermit & & & & 0.03 & & & & 0.02 \\
\hline Phaethornis pretrei (Lesson \& Delattre, 1839) & Planalto Hermit & & XC400263 & & 0.05 & & 0.03 & 0.07 & 0.07 \\
\hline Eupetomena macroura (Gmelin, 1788) & $\begin{array}{l}\text { Swallow-tailed } \\
\text { Hummingbird }\end{array}$ & & & & & & & 0.03 & \\
\hline Colibri serrirostris (Vieillot, 1816) & White-vented Violetear & & XC400255 & WA2730248 & 0.03 & & & 0.10 & \\
\hline Anthracothorax nigricollis (Vieillot, 1817) & Black-throated Mango & & & WA2731331 & 0.16 & & & & 0.02 \\
\hline Lophornis gouldii (Lesson, 1832) VU, VU* & Dot-eared Coquette & & & & 0.03 & & & & \\
\hline Chlorostilbon lucidus (Shaw, 1812) & Glittering-bellied Emerald & & & WA2741411 & 0.03 & & & 0.03 & \\
\hline Thalurania furcata (Gmelin, 1788) & Fork-tailed Woodnymph & & XC387958 & WA2723261 & 0.66 & 0.58 & 0.03 & 0.17 & 0.09 \\
\hline Hylocharis cyanus (Vieillot, 1818) & White-chinned Sapphire & & XC400341 & & 0.74 & & & & \\
\hline Hylocharis chrysura (Shaw, 1812) & Gilded Hummingbird & 4173 & & & 0.05 & 0.08 & & & \\
\hline Amazilia fimbriata (Gmelin, 1788) & Glittering-throated Emerald & & & WA2249328 & & & 0.03 & & 0.02 \\
\hline $\begin{array}{l}\text { Heliomaster longirostris (Audebert \& Vieillot, } \\
\text { 1801) }\end{array}$ & Long-billed Starthroat & & & ML87944881 & & 0.08 & & & \\
\hline Heliomaster furcifer (Shaw, 1812) & Blue-tufted Starthroat & & & & & & & 0.03 & \\
\hline Calliphlox amethystina (Boddaert, 1783) & Amethyst Woodstar & & XC400325 & WA2723264 & 0.39 & & & & \\
\hline Trogoniformes & & & & & & & & & \\
\hline Trogonidae & & & & & & & & & \\
\hline
\end{tabular}




\begin{tabular}{|c|c|c|c|c|c|c|c|c|c|}
\hline \multirow[b]{2}{*}{ Taxon name } & \multirow[b]{2}{*}{ English name } & \multicolumn{3}{|c|}{ Vouchers } & \multicolumn{5}{|c|}{ Area } \\
\hline & & Specimen & Voice & Photo & $\begin{array}{c}\text { A } \\
\text { (FO) } \\
T \\
\end{array}$ & $\begin{array}{c}\text { A } \\
\text { (FO) } \\
\text { M }\end{array}$ & $\begin{array}{c}\text { B } \\
\text { (FO) }\end{array}$ & $\begin{array}{c}\mathrm{C} \\
\text { (FO) }\end{array}$ & $\begin{array}{c}\text { D } \\
\text { (FO) }\end{array}$ \\
\hline Trogon melanurus Swainson, 1838 & Black-tailed Trogon & & XC400238 & WA2739658 & 0.61 & & & & 0.07 \\
\hline Trogon viridis Linnaeus, 1766 & Green-backed Trogon & & XC400212 & WA2404272 & 0.16 & & & & 0.02 \\
\hline Trogon curucui Linnaeus, 1766 & Blue-crowned Trogon & 4165 & & WA2720942 & 0.89 & 0.80 & 0.06 & 0.10 & 0.15 \\
\hline Trogon collaris Vieillot, 1817 & Collared Trogon & & XC400216 & WA2760486 & 0.16 & & & & \\
\hline \multicolumn{10}{|l|}{ Coraciiformes } \\
\hline \multicolumn{10}{|l|}{ Alcedinidae } \\
\hline Megaceryle torquata (Linnaeus, 1766) & Ringed Kingfisher & & XC387973 & & 0.03 & & & & \\
\hline Chloroceryle amazona (Latham, 1790) & Amazon Kingfisher & & & WA2723263 & 0.08 & & & & \\
\hline Chloroceryle aenea (Pallas, 1764) & American Pygmy Kingfisher & & & & & & & & 0.02 \\
\hline Chloroceryle americana (Gmelin, 1788) & Green Kingfisher & & & ML88502091 & 0.03 & 0.08 & & & \\
\hline Chloroceryle inda (Linnaeus, 1766) & Green-and-rufous Kingfisher & & & ML87937381 & 0.03 & 0.08 & & & \\
\hline \multicolumn{10}{|l|}{ Momotidae } \\
\hline Momotus momota (Linnaeus, 1766) & Amazonian Motmot & & XC400340 & WA2733665 & 0.82 & 0.25 & 0.16 & 0.07 & 0.22 \\
\hline \multicolumn{10}{|l|}{ Galbuliformes } \\
\hline \multicolumn{10}{|l|}{ Galbulidae } \\
\hline Galbula ruficauda Cuvier, 1816 & Rufous-tailed Jacamar & & & WA2470881 & 0.18 & & & & 0.4 \\
\hline Bucconidae & & & & & & & & & \\
\hline Notharchus tectus (Boddaert, 1783) & Pied Puffbird & & & WA2880809 & & & & & \\
\hline Bucco tamatia Gmelin, 1788 & Spotted Puffbird & & & WA2737792 & 0.05 & & & & \\
\hline Nystalus striolatus (Pelzeln, 1856) & Natterer's Striolated Puffbird & & & WA2272011 & 0.03 & & & & \\
\hline Nystalus chacuru (Vieillot, 1816) & White-eared Puffbird & & XC400265 & & & & 0.09 & 0.03 & \\
\hline Nystalus maculatus (Gmelin, 1788) & Spot-backed Puffbird & & XC400318 & & 0.37 & 0.25 & & & \\
\hline Nonnula ruficapilla (Tschudi, 1844) & Rufous-capped Nunlet & & XC404457 & WA2733668 & 0.03 & & & & 0.02 \\
\hline Monasa nigrifrons (Spix, 1824) & Black-fronted Nunbird & & XC404455 & WA2720948 & 0.89 & 0.08 & 0.06 & & 0.24 \\
\hline Chelidoptera tenebrosa (Pallas, 1782) & Swallow-winged Puffbird & & XC401100 & & & & & & \\
\hline Piciformes & & & & & & & & & \\
\hline Ramphastidae & & & & & & & & & \\
\hline Ramphastos toco Statius Müller, 1776 & Toco Toucan & & XC404588 & WA2733684 & 0.29 & & 0.09 & 0.07 & 0.02 \\
\hline Ramphastos tucanus Linnaeus, 1758 & White-throated Toucan & & XC402246 & & & & & & \\
\hline Pteroglossus inscriptus Swainson, 1822 & Lettered Aracari & & XC400207 & WA2739647 & 0.16 & & 0.06 & & 0.02 \\
\hline Pteroglossus castanotis Gould, 1834 & Chestnut-eared Aracari & & XC400328 & WA2742653 & 0.61 & 0.08 & 0.09 & 0.03 & 0.09 \\
\hline Picidae & & & & & & & & & \\
\hline Picumnus albosquamatus d'Orbigny, 1840 & White-wedged Piculet & & & & 0.05 & & & & \\
\hline Melanerpes candidus (Otto, 1796) & White Woodpecker & & & WA2850102 & 0.13 & & & 0.03 & \\
\hline Melanerpes cruentatus (Boddaert, 1783) & Yellow-tufted Woodpecker & & XC401101 & & & & 0.03 & & 0.22 \\
\hline Veniliornis affinis (Swainson, 1821) & Red-stained Woodpecker & & XC402243 & & 0.05 & & & & 0.07 \\
\hline Veniliornis passerinus (Linnaeus, 1766) & Little Woodpecker & & & & 0.08 & & & & 0.4 \\
\hline Piculus chrysochloros (Vieillot, 1818) & Golden-green Woodpecker & & & & & & & & 0.02 \\
\hline Colaptes melanochloros (Gmelin, 1788) & Green-barred Woodpecker & & XC400270 & & & & & 0.10 & \\
\hline Colaptes campestris (Vieillot, 1818) & Campo Flicker & & & & & & & 0.17 & \\
\hline Celeus torquatus (Boddaert, 1783) & Ringed Woodpecker & & XC400346 & & 0.08 & & & & \\
\hline Celeus lugubris (Malherbe, 1851) & Pale-crested Woodpecker & & & WA2470864 & 0.34 & 0.08 & 0.03 & 0.03 & 0.07 \\
\hline Dryocopus lineatus (Linnaeus, 1766) & Lineated Woodpecker & & & WA2730247 & 0.08 & & & 0.03 & \\
\hline Campephilus rubricollis (Boddaert, 1783) & Red-necked Woodpecker & & XC404575 & WA2737747 & 0.34 & & & & 0.02 \\
\hline Campephilus melanoleucos (Gmelin, 1788) & $\begin{array}{l}\text { Crimson-crested } \\
\text { Woodpecker }\end{array}$ & & & & 0.16 & & & & 0.07 \\
\hline Cariamiformes & & & & & & & & & \\
\hline Cariamidae & & & & & & & & & \\
\hline Cariama cristata (Linnaeus, 1766) & Red-legged Seriema & & XC404581 & WA2733680 & 0.03 & & 0.09 & 0.13 & \\
\hline Falconiformes & & & & & & & & & \\
\hline Falconidae & & & & & & & & & \\
\hline Caracara plancus (Miller, 1777) & Southern Caracara & & & & & & & 0.03 & \\
\hline Herpetotheres cachinnans (Linnaeus, 1758) & Laughing Falcon & & & WA2737948 & 0.08 & & 0.06 & 0.03 & \\
\hline Micrastur ruficollis (Vieillot, 1817) & Barred Forest-Falcon & & XC387980 & & 0.45 & & 0.03 & & 0.02 \\
\hline Micrastur semitorquatus (Vieillot, 1817) & Collared Forest-Falcon & & XC400213 & & 0.03 & & & & 0.02 \\
\hline Falco sparverius Linnaeus, 1758 & American Kestrel & & & & & & & & 0.02 \\
\hline Falco rufigularis Daudin, 1800 & Bat Falcon & & & WA2720926 & & & & & \\
\hline Psittaciformes & & & & & & & & & \\
\hline Psittacidae & & & & & & & & & \\
\hline Anodorhynchus hyacinthinus (Latham, 1790) VU & Hyacinth Macaw & & & & & & & & \\
\hline Ara ararauna (Linnaeus, 1758) & Blue-and-yellow Macaw & & XC400211 & & 0.16 & & & & \\
\hline Ara chloropterus Gray, 1859 & Red-and-green Macaw & & & WA2737754 & 0.18 & & & 0.10 & \\
\hline Orthopsittaca manilatus (Boddaert, 1783) & Red-bellied Macaw & & & & 0.03 & & & & \\
\hline Primolius auricollis (Cassin, 1853) & Yellow-collared Macaw & & & & 0.08 & & & & 0.02 \\
\hline Diopsittaca nobilis (Linnaeus, 1758) & Red-shouldered Macaw & & XC400321 & & 0.92 & & 0.16 & 0.27 & 0.09 \\
\hline Psittacara leucophthalmus (Statius Müller, 1776) & White-eyed Parakeet & & & & 0.03 & & 0.06 & & 0.02 \\
\hline Aratinga weddellii (Deville, 1851) & Dusky-headed Parakeet & & XC400312 & & & & & & 0.22 \\
\hline Eupsittula aurea (Gmelin, 1788) & Peach-fronted Parakeet & & XC387976 & WA2731325 & 0.08 & & 0.28 & 0.30 & 0.07 \\
\hline Brotogeris chiriri (Vieillot, 1818) & Yellow-chevroned Parakeet & & XC387977 & WA2722019 & 0.18 & & 0.25 & 0.20 & 0.09 \\
\hline Pionus menstruus (Linnaeus, 1766) & Blue-headed Parrot & & XC404583 & WA2858459 & 0.05 & & & & 0.22 \\
\hline
\end{tabular}




\begin{tabular}{|c|c|c|c|c|c|c|c|c|c|}
\hline \multirow[b]{2}{*}{ Taxon name } & \multirow[b]{2}{*}{ English name } & \multicolumn{3}{|c|}{ Vouchers } & \multicolumn{5}{|c|}{ Area } \\
\hline & & Specimen & Voice & Photo & $\begin{array}{c}\text { A } \\
\text { (FO) } \\
\text { T }\end{array}$ & $\begin{array}{c}\text { A } \\
\text { (FO) } \\
M\end{array}$ & $\begin{array}{l}\text { B } \\
\text { (FO) }\end{array}$ & $\begin{array}{c}\mathrm{C} \\
\text { (FO) }\end{array}$ & $\begin{array}{c}D \\
\text { (FO) }\end{array}$ \\
\hline Amazona amazonica (Linnaeus, 1766) & Orange-winged Parrot & & XC404461 & & 0.08 & & & & \\
\hline Amazona aestiva (Linnaeus, 1758) & Turquoise-fronted Parrot & & & & & & & & \\
\hline \multicolumn{10}{|l|}{ Passeriformes } \\
\hline \multicolumn{10}{|l|}{ Thamnophilidae } \\
\hline Myrmophylax atrothorax (Boddaert, 1783) & Black-throated Antbird & & XC400233 & WA2739648 & 0.50 & 0.33 & 0.06 & & 0.50 \\
\hline Formicivora grisea (Boddaert, 1783) & White-fringed Antwren & 4185 & XC401103 & WA2858460 & 0.97 & 0.75 & & & 0.02 \\
\hline Formicivora rufa (Wied, 1831) & Rusty-backed Antwren & & XC387972 & & & & 0.19 & & \\
\hline Dysithamnus mentalis (Temminck, 1823) & Plain Antvireo & & XC400337 & & 0.68 & 0.42 & 0.03 & & 0.22 \\
\hline Herpsilochmus longirostris Pelzeln, 1868 CE & Large-billed Antwren & & XC400264 & & 0.05 & & & & \\
\hline Thamnophilus doliatus (Linnaeus, 1764) & Barred Antshrike & & XC400262 & & & & & 0.03 & \\
\hline Thamnophilus schistaceus d'Orbigny, 1835 & Plain-winged Antshrike & & XC402784 & & & & & & 0.02 \\
\hline Thamnophilus pelzelni Hellmayr, 1924 & Planalto Slaty-Antshrike & 4183 & & WA2470876 & 0.97 & 0.75 & 0.28 & 0.13 & 0.02 \\
\hline Thamnophilus aethiops Sclater, 1858 & White-shouldered Antshrike & & XC402241 & & 0.05 & & & & 0.07 \\
\hline Thamnophilus amazonicus Sclater, 1858 & Amazonian Antshrike & $4159 / 4176$ & XC400342 & & 0.13 & 0.08 & & & \\
\hline Taraba major (Vieillot, 1816) & Great Antshrike & & & & 0.03 & & & & \\
\hline Pyriglena leuconota (Spix, 1824) & White-backed Fire-eye & & XC404570 & & 0.26 & & & & 0.13 \\
\hline Hypocnemis ochrogyna Zimmer, 1932 VU* & Rondonia Warbling-Antbird & & XC400232 & & 0.05 & & 0.06 & & 0.46 \\
\hline Willisornis poecilinotus (Cabanis, 1847) & $\begin{array}{l}\text { Common Scale-backed } \\
\text { Antbird }\end{array}$ & 4175 & & & 0.21 & 0.50 & & & \\
\hline \multicolumn{10}{|l|}{ Melanopareiidae } \\
\hline Melanopareia torquata (Wied, 1831) CE & Collared Crescentchest & & XC400320 & & & & 0.06 & 0.10 & \\
\hline \multicolumn{10}{|l|}{ Dendrocolaptidae } \\
\hline Dendrocincla fuliginosa (Vieillot, 1818) & Plain-brown Woodcreeper & 4160 & XC387951 & WA2737748 & 0.66 & 0.83 & & & 0.02 \\
\hline Sittasomus griseicapillus (Vieillot, 1818) & Olivaceous Woodcreeper & & XC401106 & WA2720928 & 0.58 & 0.50 & & & \\
\hline Xiphorhynchus guttatoides (Lafresnaye, 1850) & Lafresnaye's Woodcreeper & 4179 & & WA2720943 & 0.87 & 0.50 & 0.03 & & 0.37 \\
\hline Dendroplex picus (Gmelin, 1788) & Straight-billed Woodcreeper & & & & & & & & 0.02 \\
\hline Lepidocolaptes fuscicapillus (Pelzeln, 1868) & Rondonia Woodcreeper & & & & & & & & 0.02 \\
\hline \multicolumn{10}{|l|}{ Xenopidae } \\
\hline Xenops rutilans Temminck, 1821 & Streaked Xenops & & XC400332 & & 0.08 & & & & 0.02 \\
\hline \multicolumn{10}{|l|}{ Furnariidae } \\
\hline Furnarius rufus (Gmelin, 1788) & Rufous Hornero & & & WA2733658 & & & & 0.10 & \\
\hline Synallaxis frontalis Pelzeln, 1859 & Sooty-fronted Spinetail & & XC400256 & & & & & 0.07 & \\
\hline Pipridae Rafinesque, 1815 & & & & & & & & & \\
\hline Neopelma pallescens (Lafresnaye, 1853) & Pale-bellied Tyrant-Manakin & 4167 & XC400217 & WA2737750 & 0.97 & 0.92 & 0.22 & 0.07 & \\
\hline Pipra fasciicauda Hellmayr, 1906 & Band-tailed Manakin & $4168 / 4171$ & & WA2470867 & 0.97 & 1.00 & 0.09 & & 0.30 \\
\hline Manacus manacus (Linnaeus, 1766) & White-bearded Manakin & & & WA2720932 & 0.24 & 0.67 & & & \\
\hline Machaeropterus pyrocephalus (Sclater, 1852) & Fiery-capped Manakin & & XC400210 & & 0.08 & 0.42 & & & \\
\hline Chiroxiphia pareola (Linnaeus, 1766) & Blue-backed Manakin & 4181 & XC402245 & & 0.11 & 0.08 & & & \\
\hline Antilophia galeata (Lichtenstein, 1823) CE & Helmeted Manakin & 4172 & XC400260 & WA2740293 & 0.08 & 0.17 & 0.09 & 0.20 & \\
\hline Oxyruncidae & & & & & & & & & \\
\hline Oxyruncus cristatus Swainson, 1821 & Sharpbill & & & & & & & & 0.4 \\
\hline Tityridae & & & & & & & & & \\
\hline Tityra cayana (Linnaeus, 1766) & Black-tailed Tityra & & & & 0.05 & & 0.03 & & 0.4 \\
\hline Tityra semifasciata (Spix, 1825) & Masked Tityra & & & & 0.08 & & 0.03 & & 0.02 \\
\hline Pachyramphus polychopterus (Vieillot, 1818) & White-winged Becard & & & & & 0.08 & 0.03 & & \\
\hline Cotingidae & & & & & & & & & \\
\hline Gymnoderus foetidus (Linnaeus, 1758) & Bare-necked Fruitcrow & & & & 0.03 & & & & \\
\hline Platyrinchidae & & & & & & & & & \\
\hline Platyrinchus mystaceus Vieillot, 1818 & White-throated Spadebill & & XC387974 & & & 0.08 & 0.06 & & \\
\hline Rhynchocyclidae & & & & & & & & & \\
\hline Mionectes oleagineus (Lichtenstein, 1823) & Ochre-bellied Flycatcher & & XC387952 & & 0.18 & 0.67 & 0.03 & & \\
\hline Leptopogon amaurocephalus Tschudi, 1846 & Sepia-capped Flycatcher & 4169 & XC404459 & WA2858466 & 0.63 & 0.83 & & & 0.15 \\
\hline Corythopis delalandi (Lesson, 1830) & Southern Antpipit & 4177 & XC387945 & & 0.95 & 0.75 & & 0.03 & \\
\hline Tolmomyias sulphurescens (Spix, 1825) & Yellow-olive Flycatcher & & & & 0.21 & & & & \\
\hline Tolmomyias assimilis (Pelzeln, 1868) & Yellow-margined Flycatcher & & XC402239 & & 0.16 & & & & 0.07 \\
\hline Tolmomyias flaviventris (Wied, 1831) & Yellow-breasted Flycatcher & 4184 & XC400345 & & 0.53 & 0.33 & 0.03 & & \\
\hline $\begin{array}{l}\text { Myiornis ecaudatus (d'Orbigny \& Lafresnaye, } \\
1837 \text { ) }\end{array}$ & Short-tailed Pygmy-Tyrant & & XC402237 & & & & & & 0.02 \\
\hline Hemitriccus minor (Snethlage, 1907) & Snethlage's Tody-Tyrant & & XC402235 & & 0.08 & & & & 0.26 \\
\hline Hemitriccus flammulatus Berlepsch, 1901 & Flammulated Pygmy-Tyrant & 4186 & & WA2850104 & 0.71 & 0.25 & & & \\
\hline Hemitriccus striaticollis (Lafresnaye, 1853) & Stripe-necked Tody-Tyrant & & XC404453 & WA2720944 & 0.89 & 0.33 & & & \\
\hline $\begin{array}{l}\text { Hemitriccus margaritaceiventer (d'Orbigny \& } \\
\text { Lafresnaye, 1837) }\end{array}$ & Pearly-vented Tody-tyrant & & XC400314 & & & & 0.19 & 0.17 & \\
\hline Tyrannidae & & & & & & & & & \\
\hline Hirundinea ferruginea (Gmelin, 1788) & Cliff Flycatcher & & & WA2730241 & & & & 0.03 & \\
\hline Ornithion inerme Hartlaub, 1853 & White-lored Tyrannulet & & XC402234 & & 0.08 & & & & \\
\hline Camptostoma obsoletum (Temminck, 1824) & $\begin{array}{l}\text { Southern Beardless- } \\
\text { Tyrannulet }\end{array}$ & & XC404563 & WA2720935 & 0.66 & 0.08 & 0.09 & 0.17 & 0.02 \\
\hline Elaenia flavogaster (Thunberg, 1822) & Yellow-bellied Elaenia & & XC392827 & & 0.03 & & 0.06 & 0.23 & \\
\hline Elaenia chilensis Hellmayr, 1927 & Chilean Elaenia & $4166 / 4170$ & & & & 0.08 & & & \\
\hline
\end{tabular}




\begin{tabular}{|c|c|c|c|c|c|c|c|c|c|}
\hline \multirow[b]{2}{*}{ Taxon name } & \multirow[b]{2}{*}{ English name } & \multicolumn{3}{|c|}{ Vouchers } & \multicolumn{5}{|c|}{ Area } \\
\hline & & Specimen & Voice & Photo & $\begin{array}{c}A \\
\text { (FO) } \\
T\end{array}$ & $\begin{array}{c}\text { A } \\
\text { (FO) } \\
\text { M }\end{array}$ & $\begin{array}{c}B \\
\text { (FO) }\end{array}$ & $\begin{array}{c}\mathrm{C} \\
\text { (FO) }\end{array}$ & $\begin{array}{c}D \\
\text { (FO) }\end{array}$ \\
\hline Elaenia parvirostris Pelzeln, 1868 & Small-billed Elaenia & 4187 & & & & 0.08 & 0.13 & 0.07 & \\
\hline Elaenia cristata Pelzeln, 1868 & Plain-crested Elaenia & & & & 0.08 & & 0.06 & 0.07 & \\
\hline Elaenia chiriquensis Lawrence, 1865 & Lesser Elaenia & 4182 & XC392825 & & 0.03 & 0.17 & 0.09 & 0.07 & \\
\hline Myiopagis gaimardii (d'Orbigny, 1839) & Forest Elaenia & & XC404458 & & 0.92 & 0.50 & 0.03 & & 0.22 \\
\hline Myiopagis caniceps (Swainson, 1835) & Gray Elaenia & & XC404681 & & 0.03 & & & & \\
\hline Phaeomyias murina (Spix, 1825) & Mouse-colored Tyrannulet & & XC400327 & & 0.29 & & 0.09 & 0.10 & \\
\hline Attila bolivianus Lafresnaye, 1848 & Dull-capped Attila & & XC400235 & WA2858509 & 0.53 & 0.33 & & 0.03 & \\
\hline Legatus leucophaius (Vieillot, 1818) & Piratic Flycatcher & & XC401102 & & 0.18 & 0.08 & & & 0.02 \\
\hline $\begin{array}{l}\text { Myiarchus tuberculifer (d'Orbigny \& Lafresnaye, } \\
\text { 1837) }\end{array}$ & Dusky-capped Flycatcher & & XC400219 & WA2732376 & 0.63 & 0.08 & & & 0.17 \\
\hline Myiarchus swainsoni Cabanis \& Heine, 1859 & Swainson's Flycatcher & 4178 & & & 0.24 & 0.25 & 0.03 & & \\
\hline Myiarchus ferox (Gmelin, 1789) & Short-crested Flycatcher & & XC404569 & & 0.26 & & 0.03 & & \\
\hline Myiarchus tyrannulus (Statius Müller, 1776) & Brown-crested Flycatcher & & XC404568 & & 0.42 & 0.25 & 0.09 & 0.10 & \\
\hline Sirystes sibilator (Vieillot, 1818) & Sibilant Sirystes & & XC400206 & & 0.61 & 0.08 & 0.03 & & 0.13 \\
\hline Rhytipterna simplex (Lichtenstein, 1823) & Grayish Mourner & & XC400237 & & 0.26 & & & & \\
\hline Casiornis rufus (Vieillot, 1816) & Rufous Casiornis & & XC404454 & WA2720927 & 0.76 & 0.50 & 0.19 & 0.13 & 0.4 \\
\hline Pitangus sulphuratus (Linnaeus, 1766) & Great Kiskadee & & & WA2722018 & & & & 0.17 & \\
\hline Myiodynastes maculatus (Statius Müller, 1776) & Streaked Flycatcher & & & WA2730242 & 0.24 & 0.08 & 0.03 & 0.10 & 0.02 \\
\hline Megarynchus pitangua (Linnaeus, 1766) & Boat-billed Flycatcher & & XC400313 & & 0.21 & & 0.09 & 0.10 & 0.09 \\
\hline Myiozetetes cayanensis (Linnaeus, 1766) & Rusty-margined Flycatcher & & XC387956 & WA2722295 & 0.63 & 0.08 & 0.03 & 0.10 & 0.4 \\
\hline Tyrannus melancholicus Vieillot, 1819 & Tropical Kingbird & & & WA2737751 & 0.08 & 0.08 & 0.06 & 0.13 & \\
\hline Tyrannus savana Daudin, 1802 & Fork-tailed Flycatcher & & & WA2722020 & & & 0.03 & 0.10 & \\
\hline $\begin{array}{l}\text { Griseotyrannus aurantioatrocristatus (d'Orbigny } \\
\text { \& Lafresnaye, 1837) }\end{array}$ & Crowned Slaty Flycatcher & & & WA2730238 & & & & 0.03 & \\
\hline Empidonomus varius (Vieillot, 1818) & Variegated Flycatcher & & XC401104 & & 0.32 & 0.25 & 0.03 & 0.07 & \\
\hline Myiophobus fasciatus (Statius Müller, 1776) & Bran-colored Flycatcher & & & WA2742649 & & & 0.03 & 0.03 & \\
\hline Pyrocephalus rubinus (Boddaert, 1783) & Vermilion Flycatcher & & & WA2722017 & & & & 0.03 & \\
\hline Cnemotriccus fuscatus (Wied, 1831) & Fuscous Flycatcher & & XC400343 & WA2720929 & 0.74 & 0.83 & 0.03 & 0.07 & \\
\hline Lathrotriccus euleri (Cabanis, 1868) & Euler's Flycatcher & & XC404452 & WA2732380 & 0.61 & 0.42 & & & \\
\hline Xolmis cinereus (Vieillot, 1816) & Gray Monjita & & & WA2733679 & & & & 0.07 & \\
\hline \multicolumn{10}{|l|}{ Vireonidae } \\
\hline Cyclarhis gujanensis (Gmelin, 1789) & $\begin{array}{l}\text { Rufous-browed } \\
\text { Peppershrike }\end{array}$ & & & WA2731326 & 0.95 & 0.17 & 0.28 & 0.30 & 0.13 \\
\hline Vireo olivaceus (Linnaeus, 1766) & Red-eyed Vireo & 4158 & & & 0.05 & 0.17 & & & \\
\hline Vireo chivi (Vieillot, 1817) & Chivi Vireo & & XC387946 & WA2722314 & 0.58 & 0.50 & 0.06 & 0.03 & \\
\hline \multicolumn{10}{|l|}{ Corvidae } \\
\hline Cyanocorax cyanomelas (Vieillot, 1818) & Purplish Jay & & XC400317 & & 0.03 & & 0.19 & & 0.02 \\
\hline Cyanocorax cristatellus (Temminck, 1823) CE & Curl-crested Jay & & XC400261 & WA2730240 & 0.05 & & & 0.23 & \\
\hline \multicolumn{10}{|l|}{ Hirundinidae } \\
\hline Stelgidopteryx ruficollis (Vieillot, 1817) & $\begin{array}{l}\text { Southern Rough-winged } \\
\text { Swallow }\end{array}$ & & & WA2722297 & 0.21 & & & 0.03 & 0.09 \\
\hline Progne tapera (Vieillot, 1817) & Brown-chested Martin & & & WA2741410 & & & & 0.10 & \\
\hline Progne chalybea (Gmelin, 1789) & Gray-breasted Martin & & & WA2739645 & & & & 0.07 & \\
\hline \multicolumn{10}{|l|}{ Troglodytidae } \\
\hline Troglodytes musculus Naumann, 1823 & Southern House Wren & & XC400319 & WA2741418 & & & 0.03 & 0.10 & \\
\hline Campylorhynchus turdinus (Wied, 1831) & Thrush-like Wren & & & & 0.03 & & & & \\
\hline Pheugopedius genibarbis (Swainson, 1838) & Moustached Wren & & XC402824 & & 0.03 & & & & 0.11 \\
\hline \multicolumn{10}{|l|}{ Polioptilidae } \\
\hline Polioptila dumicola (Vieillot, 1817) & Masked Gnatcatcher & & & & & & & & \\
\hline \multicolumn{10}{|l|}{ Turdidae } \\
\hline Catharus fuscescens (Stephens, 1817) & Veery & 4161 & & & & 0.08 & & & \\
\hline Turdus leucomelas Vieillot, 1818 & Pale-breasted Thrush & & XC387949 & WA3050241 & 0.95 & 0.50 & 0.13 & 0.17 & 0.4 \\
\hline Turdus hauxwelli Lawrence, 1869 & Hauxwell's Thrush & $4157 / 4163$ & XC402233 & WA2850093 & 0.39 & 0.67 & & & 0.13 \\
\hline Turdus rufiventris Vieillot, 1818 & Rufous-bellied Thrush & & XC400267 & WA2741419 & & & 0.03 & 0.07 & 0.02 \\
\hline Turdus amaurochalinus Cabanis, 1850 & Creamy-bellied Thrush & 4180 & & WA2866278 & 0.08 & 0.25 & 0.09 & 0.07 & 0.02 \\
\hline Mimidae & & & & & & & & & \\
\hline Mimus saturninus (Lichtenstein, 1823) & Chalk-browed Mockingbird & & XC387969 & WA2731343 & & & 0.13 & 0.20 & \\
\hline Passerellidae & & & & & & & & & \\
\hline Zonotrichia capensis (Statius Müller, 1776) & Rufous-collared Sparrow & & XC400258 & WA2733669 & & & & 0.13 & \\
\hline Ammodramus humeralis (Bosc, 1792) & Grassland Sparrow & & & WA2730239 & & & & 0.07 & \\
\hline Arremon taciturnus (Hermann, 1783) & Pectoral Sparrow & $4162 / 4174$ & XC400230 & & 0.66 & 0.67 & & & 0.11 \\
\hline Arremon flavirostris Swainson, 1838 & Saffron-billed Sparrow & & XC404275 & & & & & 0.07 & \\
\hline Parulidae & & & & & & & & & \\
\hline Geothlypis aequinoctialis (Gmelin, 1789) & Masked Yellowthroat & & & & & & & 0.03 & \\
\hline Basileuterus culicivorus (Deppe, 1830) & Golden-crowned Warbler & & XC387944 & WA2723262 & 1.00 & 0.83 & 0.13 & 0.10 & 0.37 \\
\hline Myiothlypis flaveola Baird, 1865 & Flavescent Warbler & & XC401105 & WA2866279 & 0.97 & 0.75 & 0.28 & 0.10 & 0.13 \\
\hline Icteridae & & & & & & & & & \\
\hline Cacicus cela (Linnaeus, 1758) & Yellow-rumped Cacique & & & WA2858465 & 0.16 & & 0.06 & 0.03 & 0.11 \\
\hline Icterus pyrrhopterus (Vieillot, 1819) & Variable Oriole & & XC387981 & WA2733656 & 0.11 & & 0.03 & & 0.07 \\
\hline Icterus croconotus (Wagler, 1829) & Orange-backed Troupial & & XC400316 & WA2866291 & & & & & 0.4 \\
\hline
\end{tabular}




\begin{tabular}{|c|c|c|c|c|c|c|c|c|c|}
\hline \multirow[b]{2}{*}{ Taxon name } & \multirow[b]{2}{*}{ English name } & \multicolumn{3}{|c|}{ Vouchers } & \multicolumn{5}{|c|}{ Area } \\
\hline & & Specimen & Voice & Photo & $\begin{array}{c}A \\
\text { (FO) } \\
\text { T }\end{array}$ & $\begin{array}{c}\text { A } \\
\text { (FO) } \\
\text { M }\end{array}$ & $\begin{array}{l}\text { B } \\
\text { (FO) }\end{array}$ & $\begin{array}{c}\mathrm{C} \\
\text { (FO) }\end{array}$ & $\begin{array}{l}\text { D } \\
\text { (FO) }\end{array}$ \\
\hline Gnorimopsar chopi (Vieillot, 1819) & Chopi Blackbird & & & WA2730245 & & & & 0.17 & \\
\hline Molothrus bonariensis (Gmelin, 1789) & Shiny Cowbird & & & WA2732455 & & & & 0.07 & \\
\hline \multicolumn{10}{|l|}{ Thraupidae } \\
\hline Neothraupis fasciata (Lichtenstein, 1823) CE NT & White-banded Tanager & & XC387979 & & & & 0.06 & 0.10 & \\
\hline Cissopis leverianus (Gmelin, 1788) & Magpie Tanager & & XC400336 & WA2737753 & 0.05 & & & & 0.07 \\
\hline Schistochlamys melanopis (Latham, 1790) & Black-faced Tanager & & XC400180 & WA2739641 & 0.37 & 0.08 & 0.19 & 0.27 & \\
\hline Tangara mexicana (Linnaeus, 1766) & Turquoise Tanager & & & WA2858467 & 0.05 & & & & \\
\hline Tangara sayaca (Linnaeus, 1766) & Sayaca Tanager & & XC400257 & WA2866107 & 0.18 & & 0.22 & 0.37 & \\
\hline Tangara palmarum (Wied, 1821) & Palm Tanager & & & WA2741415 & 0.37 & 0.08 & 0.03 & 0.03 & \\
\hline Tangara cayana (Linnaeus, 1766) & Burnished-buff Tanager & & & WA2858468 & & & 0.06 & 0.10 & \\
\hline Sicalis citrina Pelzeln, 1870 & Stripe-tailed Yellow-Finch & & XC400311 & & & & & 0.10 & \\
\hline Hemithraupis guira (Linnaeus, 1766) & Guira Tanager & & XC387978 & WA2731327 & & & 0.09 & & \\
\hline Volatinia jacarina (Linnaeus, 1766) & Blue-black Grassquit & & & WA2732453 & & & & 0.03 & \\
\hline Eucometis penicillata (Spix, 1825) & Gray-headed Tanager & & XC400222 & & 0.16 & 0.42 & & & 0.22 \\
\hline $\begin{array}{l}\text { Coryphospingus cucullatus (Statius Müller, } \\
\text { 1776) }\end{array}$ & Red-crested Finch & & & WA2730246 & & 0.08 & 0.06 & 0.07 & \\
\hline Lanio luctuosus (d'Orbigny \& Lafresnaye, 1837) & White-shouldered Tanager & & & WA2858508 & 0.21 & 0.08 & & & 0.07 \\
\hline Lanio cristatus (Linnaeus, 1766) & Flame-crested Tanager & & XC404462 & & 0.03 & & & & \\
\hline Tachyphonus rufus (Boddaert, 1783) & White-lined Tanager & & XC404456 & WA2877693 & 0.55 & 0.42 & 0.06 & 0.13 & 0.07 \\
\hline Ramphocelus carbo (Pallas, 1764) & Silver-beaked Tanager & & & WA2858501 & 0.82 & 0.08 & & 0.10 & \\
\hline Tersina viridis (Illiger, 1811) & Swallow Tanager & & XC400344 & WA2733661 & 0.29 & & 0.03 & 0.07 & \\
\hline Cyanerpes cyaneus (Linnaeus, 1766) & Red-legged Honeycreeper & & & WA2742652 & 0.61 & 0.42 & 0.09 & 0.07 & 0.02 \\
\hline Dacnis cayana (Linnaeus, 1766) & Blue Dacnis & & XC400326 & WA2470866 & 0.58 & 0.17 & 0.13 & 0.10 & 0.4 \\
\hline Sporophila plumbea (Wied, 1830) & Plumbeous Seedeater & & & WA2730249 & & & & 0.07 & \\
\hline Sporophila cf. nigricollis (Vieillot, 1823) & Yellow-bellied Seedeater & 4164 & & & & 0.08 & & & \\
\hline Sporophila caerulescens (Vieillot, 1823) & Double-collared Seedeater & & & WA2272170 & & & & & \\
\hline Sporophila leucoptera (Vieillot, 1817) & White-bellied Seedeater & & & & & & & 0.03 & \\
\hline Saltatricula atricollis (Vieillot, 1817) CE & Black-throated Saltator & & XC387971 & WA2742646 & & & 0.25 & 0.17 & \\
\hline Saltator maximus (Statius Müller, 1776) & Buff-throated Saltator & & XC400228 & WA2866114 & 0.84 & 0.50 & 0.06 & & 0.13 \\
\hline Saltator coerulescens Vieillot, 1817 & Grayish Saltator & & & & & & 0.03 & & \\
\hline Saltator similis d'Orbigny \& Lafresnaye, 1837 & Green-winged Saltator & & XC387961 & & & & 0.03 & & \\
\hline Cypsnagra hirundinacea (Lesson, 1831) CE & White-rumped Tanager & & XC387970 & WA2739644 & & & 0.06 & 0.03 & \\
\hline \multicolumn{10}{|l|}{ Cardinalidae } \\
\hline Piranga flava (Vieillot, 1822) & Hepatic Tanager & & XC387975 & WA2731328 & & & 0.06 & & \\
\hline \multicolumn{10}{|l|}{ Fringillidae } \\
\hline Euphonia chlorotica (Linnaeus, 1766) & Purple-throated Euphonia & & XC404573 & WA2760484 & 0.95 & 0.08 & 0.34 & 0.17 & 0.15 \\
\hline $\begin{array}{l}\text { Euphonia laniirostris d'Orbigny \& Lafresnaye, } \\
1837\end{array}$ & Thick-billed Euphonia & & & WA2732379 & 0.47 & 0.25 & & & 0.4 \\
\hline
\end{tabular}

New records for Estação Ecológica da Serra das Araras

Agamia agami (Gmelin, 1789) Figure 3A

We recorded a single individual in September 2016, foraging along the Camarinha River, near the EESA headquarters, in Area A. It was readily identified by its multicolored plumage, according to Sick (1997).

\section{Cathartes melambrotus Wetmore, 1964}

We recorded this species soaring on thermal currents over a semideciduous seasonal forest. Identified by black primaries contrasting with grayish secondaries and by the typical silhouette.

\section{Harpagus bidentatus (Latham, 1790) Figure 3B}

We recorded the species twice, the first time randomly in January 2017, as the bird soared on a thermal above the EESA headquarters, and the second in February 2017, when an individual was perched on a tree canopy. Both times the species was identified by the grayish top and sides of head, rufous breast, belly and thighs barred with white and bipartite stripe on throat (Sick 1997).

\section{Harpagus diodon (Temminck, 1823)}

We recorded this species in September 2016, ascending on a thermal, in Area A. Identified by its rufous thighs and upper wing coverts and light gray breast, which differentiates it from $H$. bidentatus.

Accipiter superciliosus (Linnaeus, 1766) Figure 3C

Our first contact with this species was random, on the border of a seasonal forest near EESA headquarters in October 2016. During the transects we had 2 more contacts, in February and August 2017. The species was identified in the field by its relatively short tail, slate gray upper parts, and white underparts and thighs thickly barred with narrow buffish stripes (Sick 1997).

\section{Coccyzus euleri Cabanis, 1873 Figure 3D}

We recorded the species in December 2016, on the border of a semideciduous seasonal forest, while it moved on the upper middle level of the canopy. Identified in the field by the uniform earth-brown upperparts, light pearly-gray below, whitish belly and long tail. No rufous primaries, which are evident in its congener Coccyzus americanus (Payne 2018).

\section{Lurocalis semitorquatus (Gmelin, 1789)}

We recorded the species by voice, in March 2017, on the border of a semideciduous seasonal forest. 


\section{Cypseloides senex (Temminck, 1826)}

We recorded the species systematically in February and March 2017 (Area A) and in August 2017 (Area B). During the rest of the study, we made additional random contacts with this species. On many occasions, groups of individuals $(>40)$ were recorded in flight. Identified in the field by its whitish head contrasting with the rest of the body, a characteristic not seen in its congener Cypseloides fumigatus (Streubel, 1848), according to Sick (1997).

\section{Lophornis gouldii (Lesson, 1832) Figure 3E}

We recorded this species systematically in July 2017 in Area A, at an altitude of $230 \mathrm{~m}$, on the border of a riparian forest. Readily identified by its white lateral tufts with shining green extremities, according to Züchner and Boesman (2018a) and longer than the ones found in Lophornis magnificus (Vieillot, 1817) (Züchner and Boesman 2018b).

\section{Hylocharis cyanus (Vieillot, 1818)}

We recorded this species by voice several times in a semideciduous seasonal forest during the transects (FO $=0.74$ ), and also captured an individual, identified by its violet-blue head, neck and chest, white chin and blacktipped red bill (Sick 1997).

\section{Ramphastos tucanus Linnaeus 1758}

We recorded the subspecies Ramphastos $t$. cuvieri Wagler (1827) in October 2016 randomly, by voice. The bird was in a riparian forest in Area A.

\section{Veniliornis affinis (Swainson, 1821)}

We recorded this species twice, in January and August 2017, in the riparian forest in Area A. Two additional contacts took place in deciduous seasonal forests, in Area D. In one case, the contact was documented by audio recording.

\section{Celeus torquatus (Boddaert, 1783) Figure 3F}

We recorded the subspecies Celeus torquatus occidentalis (Hargitt, 1889) randomly in August 2016, in Area A. Other contacts were made systematically during the transects, in July and September 2017. The species was identified in the field by its caramel-colored head, black foreneck and chest, chestnut mantle with black barring and whitish belly with coarse black markings (Sick 1997). The male has a broad red band on the malar region (Winkler et al. 2018). In some cases, the species was identified solely by voice.

\section{Primolius auricollis (Cassin, 1853)}

We recorded a flock flying overhead in December 2016, without being able to obtain a voucher specimen. The species was readily identified by the yellow collar on the lower hindneck and pale-yellow naked face (Sick 1997).

\section{Thamnophilus schistaceus d'Orbigny, 1835}

We recorded this species systematically in Area D, on a patch of semideciduous seasonal forest in August 2017. Identified by voice while vocalizing in mid-level vegetation.
Thamnophilus aethiops Sclater, 1858 Figure 3G

We recorded this species in Area A, in January and August 2017 in semideciduous seasonal forest. We also recorded 2 individuals in Area D, in August. At all times the species was first identified by voice.

\section{Thamnophilus amazonicus Sclater, 1858 Figure $3 \mathrm{H}$}

We first recorded this species by voice in riparian forest in February 2017. We later recorded it in March, June and July of the same year. We collected 2 specimens with mist nets and deposited them at UFMT under catalog numbers 4159 and 4176.

\section{Chiroxiphia pareola (Linnaeus, 1766) Figure 4A}

We first recorded this species by several sound contacts in December 2016 and January, February and March 2017 in semideciduous seasonal forest. We also captured a female and deposited it at UFMT under catalog number 4181. According to the geographical distribution given by BirdLife International and the Handbook of the Birds of the World (2016), records for C. pareola at EESA extend the species' known geographical range.

\section{Gymnoderus foetidus (Linnaeus, 1758)}

We recorded the species systematically in September 2017, while 1 individual flew over a riparian forest. The species was identified by its black plumage, throat wattles and blue bare skin on the sides of the neck (Sick 1997).

\section{Tolmomyias assimilis (Pelzeln, 1868)}

We recorded this species in semideciduous seasonal forest in Area A in February, March, April and May 2017. We also recorded it in Area D, the northern part of the EESA. At all times, it was identified by voice. According to the geographical distribution given by BirdLife International and the Handbook of the Birds of the World (2016), records for T. assimilis at EESA extend the species' known geographical range.

\section{Tolmomyias flaviventris (Wied, 1831) Figure 4B}

We recorded the species on several occasions. A relatively common bird at EESA, with over 50\% frequency of occurrence in Area A. A specimen was collected and deposited at UFMT under catalog number 4184.

\section{Myiornis ecaudatus (d'Orbigny \& Lafresnaye, 1837)}

We recorded this species in area $\mathrm{D}$ by voice while the individual vocalized in a tree canopy.

\section{Hemitriccus minor (Snethlage, 1907)}

We first recorded this species in May 2016 in Area A, in semideciduous seasonal forest. We had several other contacts with the species in August in Area D, where it is more frequent. Identified most times by voice. According to the geographical distribution given by BirdLife International and the Handbook of the Birds of the World (2016), records for H. minor at EESA extend the species' known geographical range.

\section{Ornithion inerme Hartlaub, 1853}

We recorded the species once in March and twice in August 2017 in semideciduous seasonal forest and border 

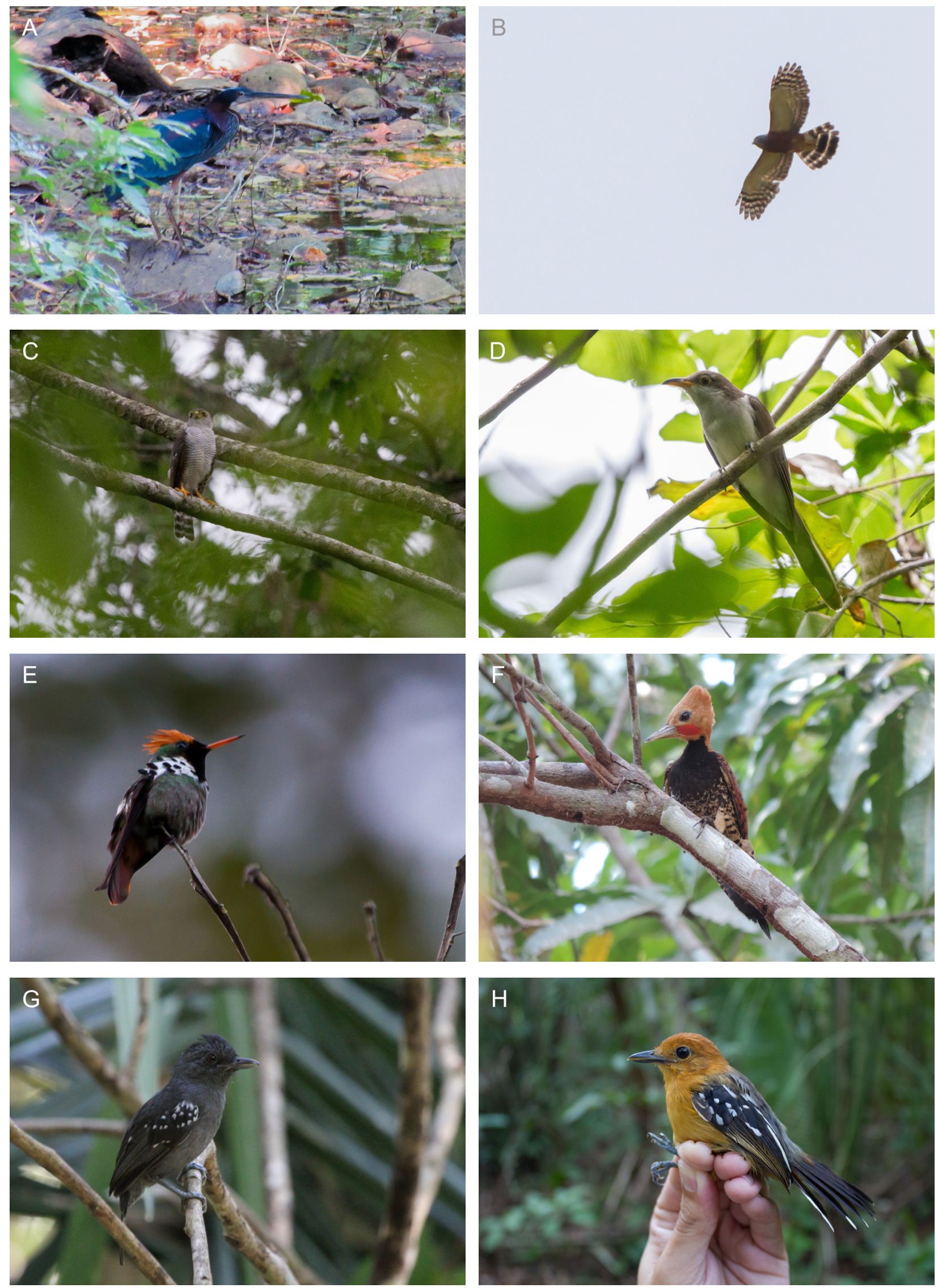

Figure 3. A. Agamia agami. B. Harpagus bidentatus. C. Accipiter superciliosus. D. Coccyzus euleri. E. Lophornis gouldii. F. Celeus torquatus. G. Thamnophilus aethiops. H. Thamnophilus amazonicus. (Photos: BDV except for Celeus torquatus, photographed by AVBF). 
areas. Identified by voice at all times. According to the geographical distribution given by BirdLife International and the Handbook of the Birds of the World (2016), records for $O$. inerme at EESA extend the species' known geographical range.

\section{Rhytipterna simplex (Lichtenstein, 1823)}

We recorded the species in April, May, June, July, August and September 2017. It was present in $26 \%$ of the repetitions in transect $\mathrm{A}$. We readily identified it by voice at all times. In the EESA it is more often heard than seen.

\section{Vireo olivaceus (Linnaeus, 1766) Figure 5A}

We captured 3 specimens with mist nets, 1 in November 2016 and 2 in January 2017. Identified by the elongated wings and the red iris. One individual was collected and deposited at UFMT under catalog number 4158. This is a Nearctic migrant that winters at EESA.

\section{Turdus hauxwelli Lawrence, 1869 Figure 4C}

We recorded this species 18 times in the Area A transects, with additional contacts in Area C. Identification was always made by this species' typical call, the surest means of field identification, given the great similarity between T. hauxwelli and T. fumigatus. Some specimens were captured with mist nets. Two specimens were collected and deposited under catalog numbers 4157 and 4163 at UFMT, where the skins were identified through comparison.

\section{Lanio cristatus (Linnaeus, 1766)}

We recorded a male of this species in semideciduous seasonal forest, but could not obtain a voucher specimen. Nevertheless, the individual was readily identified for its black plumage with yellowish buff throat and scarlet crown, as described in Sick (1997).

\section{Near Threatened species (IUCN 2017)}

\section{Rhea americana (Linnaeus, 1758)}

We recorded the species in an open area under influence of a rural zone, in Area C. Readily identified by its gray mantle forming a dorsal humpback and enveloping the whole body, except for the rear, which is white and lacks a tail (Sick 1997).

\section{Odontophorus gujanensis (Gmelin, 1789) Figure 5B}

We recorded the species in riparian forest and semideciduous seasonal forest, mostly near water bodies. Most times identified by voice. The species has reddish brown belly and bare orange eyering, which distinguishes it from its congener O. capoeira (Sick 1997).

\section{Spizaetus ornatus (Daudin, 1800) Figure 5C}

We recorded the species in Area A, associated with forest or natural border areas. We also made 1 record in Area B. The species was identified mostly by voice. Sometimes the identification was visual, by its short, rounded wings and exceptionally long tail, legs and claws. Heavily plumed legs and a crest frequently erect at the top of the head (Sick 1997).
Neothraupis fasciata (Lichtenstein, 1823) Figure 5D

We recorded the species in cerrado areas, generally at altitudes above $500 \mathrm{~m}$, many times in mixed flocks with Saltatricula atricollis (Vieillot, 1817). Identified by gray upper parts, black mask, and black wing-coverts with white band; white throat and belly, gray breast (Sick 1997).

\section{Threatened species}

\section{Tinamus tao Temminck, 1815 Figure 5E}

All records of this species were associated with riparian and seasonal forests. Identified by the bluish slate-gray back and light lead-gray belly (Sick 1997). The species is currently classified as Vulnerable at the national (Brazil 2016b) and global (IUCN 2017) levels.

\section{Crax fasciolata Spix, 1825}

We recorded the species mostly in forest environments. The male has black plumage with white belly. The tip of the tail is white, distinguishing it from its congeners (Sick 1997). Currently classified as Vulnerable at the global level (IUCN 2017).

\section{Agamia agami (Gmelin, 1789)}

Described above. Classified as Vulnerable at the global level (IUCN 2017).

\section{Lophornis gouldii (Lesson, 1832)}

Described above. Classified as Vulnerable at the national (Brazil 2016b) and global (IUCN 2017) levels.

\section{Anodorhynchus hyacinthinus (Latham, 1790)}

We recorded the species once, flying overhead. Identified by the dark blue plumage and yellow eyering, eyelid and strip at base of mandible (Sick 1997). Classified as Vulnerable at the global level (IUCN 2017).

\section{Hypocnemis ochrogyna Zimmer, 1932 Figure 5F}

We recorded this species in Areas A and D, always in forest environments. In the EESA area, it replaces geographically its congeners of the Hypocnemis cantator complex. Identified most times by its song. Classified as Vulnerable at the national level (Brazil 2016b).

\section{Cerrado endemics}

\section{Herpsilochmus longirostris Pelzeln, 1868}

We recorded this species at the border of a gallery forest, by voice.

\section{Melanopareia torquata (Wied, 1831) Figure 5G}

We recorded the species in cerrado areas, generally at altitudes above $500 \mathrm{~m}$. Identified by the black collar on the throat, white eyebrow and black sides of the head (Sick 1997).

\section{Antilophia galeata (Lichtenstein, 1823)}

We recorded the species in riparian forest areas, mostly by voice. One female was collected and deposited at UFMT under catalog number 4172.

Cyanocorax cristatellus (Temminck, 1823) 

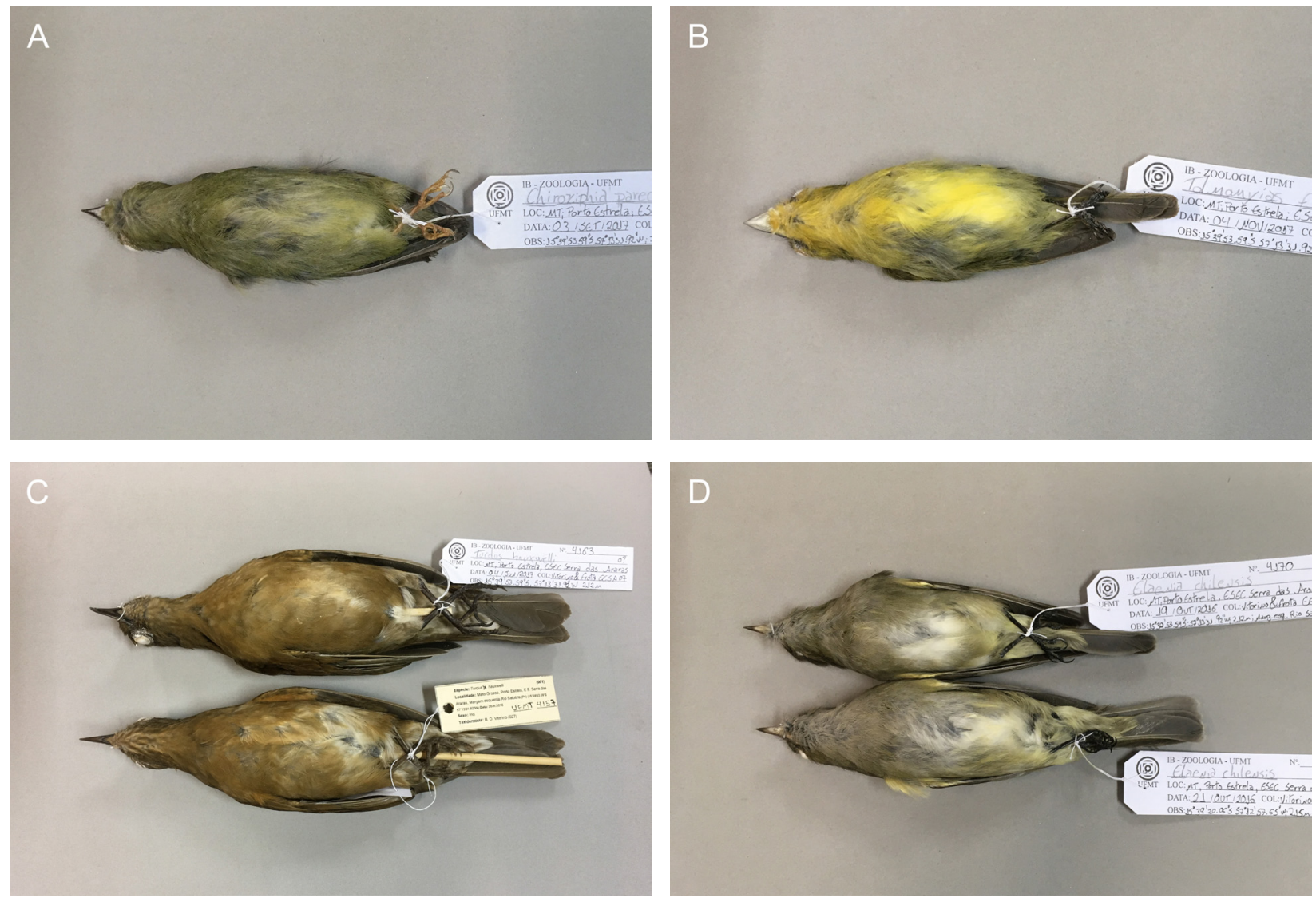

Figura 4. A. Chiroxiphia pareola. B. Tolmomyias flaviventris. C. Turdus hauxwelli. D. Elaenia chilensis. (Photos: Vitor Q. Piacentini).

We recorded the species in cerrado areas, generally at altitudes above $500 \mathrm{~m}$. Identified by its long wings and relatively short tail with dark violet-blue mantle and white belly and distal two-thirds of tail. Unmistakable due to long frontal crest, separated from the crown (Sick 1997), in addition to its distinctive call.

\section{Neothraupis fasciata (Lichtenstein, 1823)}

Described above.

\section{Cypsnagra hirundinacea (Lesson, 1831)}

We recorded the species in cerrado areas, generally at altitudes above $500 \mathrm{~m}$. Identified by black upper parts, with 2 white bands on wings and white rump. Whitish underparts, rufous throat (Sick 1997) and distinctive song.

\section{Other noteworthy records}

\section{Nystalus striolatus (Pelzeln, 1856)}

We recorded this species associated with forest environments and occasionally to transition zones. Identified by the yellowish breast with black stripes. Similar to congeners Nystalus obamai Whitney, Piacentini, Schunck, Aleixo, Sousa, Silveira \& Rego, 2013 and Nystalus torridus Bond \& Meyer de Schauensee, 1940, but geographically separated.

\section{Oxyruncus cristatus Swainson, 1821 Figure 6}

We recorded this species 3 times: once in a transition area from semideciduous seasonal forest to cerrado stricto sensu in Area A, and 2 in a seasonal forest dominated by Attalea speciosa in Area D. The individuals were readily identified by their olive green color, red iris and yellowish underparts with black spots (Brooke 2018). The photo we present in this article is the first photographic record for the species in Mato Grosso, as we ascertained after an exhaustive search in the literature, ornithological collections and virtual databanks.

\section{Elaenia chilensis Hellmayr, 1927 Figure 4E}

We captured 2 individuals, on 19 and 21 October 2016, respectively. They were collected and deposited at UFMT under catalog numbers 4170 and 4166, and identified through comparison with other specimens from the collection. Elaenia chilensis is an Austral migrant, with scarce documented records for the state of Mato Grosso. The information presented herein may contribute to understand the species' distribution and migration patterns and fill some of the existing knowledge gaps.

\section{Catharus fuscescens (Stephens, 1817) Figure 5H}

We recorded this species in riparian and semideciduous seasonal forests, where it tends to be very discreet. Identified by rufous brown upperparts, white throat and belly, and yellowish breast. Sides of neck covered with brownish spotting (Sick 1997). One specimen was captured with mist net and deposited at UFMT under catalog number 4161. In Brazil, it is a seasonal visitor from the northern hemisphere (Piacentini et al. 2015). 

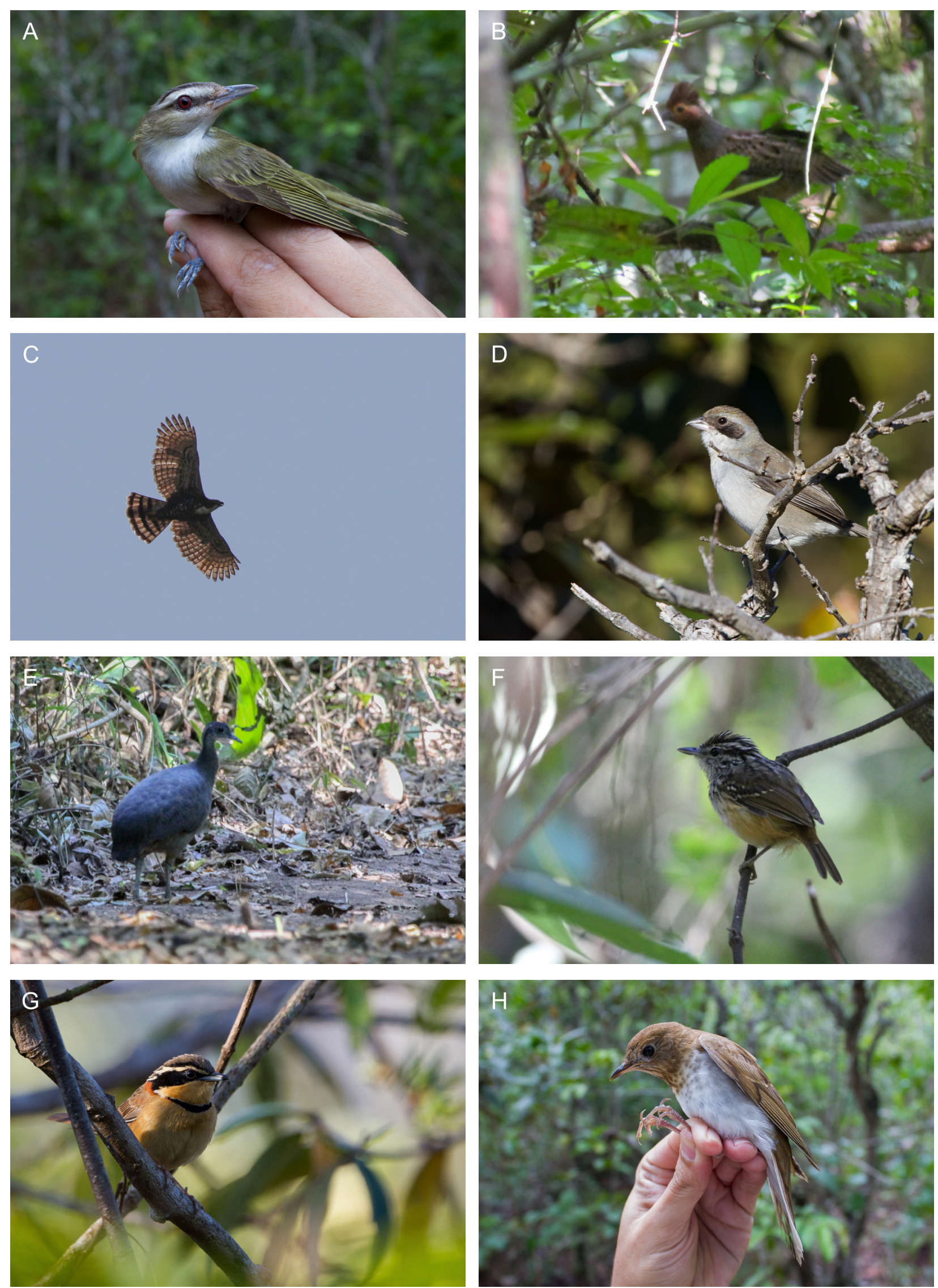

Figura 5. A. Vireo olivaceus. B. Odontophorus gujanensis. C. Spizaetus ornatus. D. Neothraupis fasciata. E. Tinamus tao. F. Hypocnemis ochrogyna. G. Melanopareia torquata. H. Catharus fuscescens. (Photos: BDV). 


\section{Birds of Estação Ecológica da Serra das Araras: review}

With the review of the bird occurrence records for EESA, we compiled a total of 458 species for the consolidated list, distributed over 27 orders and 69 families. Of these, 255 were assigned to the primary list and 203 to the secondary list (Table 3). On the tertiary list we included 7 species and 1 taxon identified only at the genus level (Table 4).

The following records placed on the secondary list deserve special attention due to their questionable nature:

\section{Columbina cyanopis (Pelzeln, 1870)}

Listed by Silva and Oniki (1988) and Valadão (2012). An extremely rare species. Occurrence records of $C$. cyanopis at EESA are based on 3 non-documented contacts. The first 2, presented by Silva and Oniki (1988), took place on 19 and 24 January 1986. The bird was identified by the distinct chestnut head and the blue markings on the wings. The third record (Valadão 2012) took place in campo rupestre, in March 2007. However, the species is mentioned in this context due to the absence of documented evidence for these 3 records and the many unsuccessful campaigns recently carried out in the region to document the species (Save Brazil 2010, Save Brazil 2011, Valadão 2012 and this article).

\section{Cypseloides fumigatus (Streubel, 1848)}

Listed by Silva and Oniki (1988) and Valadão (2012). A taxon difficult to identify, scarce in the region and which may be confused with Cypseloides senex. Cypseloides senex was recorded by us several times and does not feature in the lists by Willis and Oniki (1990) and Valadão (2012). We stress that at times we have come across individuals of genus Cypseloides without being able to identify them at the species level, so it is possible that both species occur at EESA.

\section{Isleria hauxwelli (Sclater, 1857)}

Listed by Silva and Oniki (1988) and Valadão (2012). This is a species typical of terra firme forest, and uncommon in the EESA region.

\section{Thamnophilus caerulescens Vieillot, 1816}

Listed by Silva and Oniki (1988). Uncommon in the region.

\section{Rhegmatorhina hoffmannsi (Hellmayr, 1907)}

Listed by Silva and Oniki (1988) and Valadão (2012). A species associated to terra firme forest, and uncommon in the EESA region.

\section{Pachysylvia muscicapina (Sclater \& Salvin, 1873)}

Listed by Silva and Oniki (1988). A species associated to terra firme forest and várzea, and uncommon in the EESA region.

\section{Turdus fumigatus Lichtenstein, 1823}

Listed by Willis and Oniki (1990) and Valadão (2012). Scarce in the region and similar to its congener T. hauxwelli. During our expeditions, we recorded T. hauxwelli several times and collected 2 skins. Since T. hauxwelli is not listed by Willis and Oniki (1990) or Valadão (2012), the documentation of T. fumigatus for EESA, especially for specimens collected, becomes essential.

Obtaining documented evidence for the species described above should be a priority in future studies at EESA. We also stress the importance of documenting other hitherto undocumented taxa whose presence in the EESA and in the southwest portion of the state of Mato Grosso is relevant due to their distribution pattern, irradiation and conservation: Electron platyrhynchum (Leadbeater, 1829), Microrhopias quixensis (Cornalia, 1849), Conopophaga lineata (Wied, 1831), Hylexetastes uniformis Hellmayr, 1909, Clibanornis rectirostris (Wied, 1831), Syndactyla dimidiata (Pelzeln, 1859), Synallaxis gujanensis (Gmelin, 1789), Euscarthmus rufomarginatus (Pelzeln, 1868), Culicivora caudacuta (Vieillot, 1818) and Sporophila maximiliani (Cabanis, 1851).

\section{Discussion}

In evaluating the richness of birds reported to EESA, it is observed a higher number of species recorded in relation to several areas of the Cerrado (see Valadão 2012), as well as several other protected areas in Brazil (Motta-Junior et al. 2008, Cavarzere et al. 2009, Aleixo and Guilherme 2010), with methodological reservations. Its expressive number of species places EESA among the richest protected areas in terms of bird species in Brazil (Pacheco et al. 2007, Pinheiro and Dornas 2009, Somenzari et al. 2011), especially in terms of areas not primarily covered by forest. This richness becomes even more significant when we consider the reduced size of the EESA, less than 30,000 ha.

This high bird species richness observed in EESA is associated with habitat diversity (Valadão 2012) as well as the integrity of the area. These habitats compose a mosaic comprising campo, savanna and forest areas inserted in an ecotone between Cerrado, the Amazon and the Pantanal wetland. Other studies done in ecotone areas have also evidenced high bird richness (Silveira and D'Horta 2002, Pinheiro and Dornas 2009, Somenzari et al. 2011, Lopes et al. 2016).

EESA' location, in the Upper Paraguay River, between the Plate River and the Amazon River basins, in addition to the multiple contacts between the riparian forest and other vegetation formations, favors an intense mix of avifaunal elements (Silva and Oniki 1988). Although located in the Cerrado, EESA displays a strong Amazonian influence on the bird composition in forest areas, be them riparian, lower montane, seasonal semideciduous or seasonal deciduous.

Some Amazonian species, like Myrmophylax atrothorax, and Tangara mexicana (Linnaeus, 1766), have been recorded in EESA since the first studies done in the area. Others, like Thamnophilus amazonicus and Chiroxiphia pareola, first recorded in our survey, suggest some recent 
Table 3. Consolidated list of birds recorded at Estação Ecológica da Serra das Araras, state of Mato Grosso, Brazil. Sources: 1 Silva and Oniki (1988); 2 Willis and Oniki (1990); 3 Oniki and Oliveira (2002); 4 Valadão (2012); 5 this study; $m p=$ mentioned exclusively in the management plan (Brazil 2016a); IO = Indication of Occurrence (species not documented); AD = A-level documentation (species with specimens deposited in scientific collections); $\mathrm{BD}=\mathrm{B}$-level documentation (species documented with photographs or audio recordings); $\mathrm{VU}^{*}=\mathrm{Vulnerable}$ (Brazil 2016b); $\mathrm{EN}^{*}=$ Endangered (Brazil 2016b); $C R^{*}=$ Critically Endangered (Brazil 2016b); NT = Near Threatened (IUCN 2017); VU = Vulnerable (IUCN 2017); EN = Endangered (IUCN 2017); CE = Cerrado endemic. Secondary list species in brackets. Voucher numbers given in text, Table 2 and Appendix Table A1.

\begin{tabular}{|c|c|c|c|}
\hline Taxon & English name & Source & Record type \\
\hline \multicolumn{4}{|l|}{ Rheiformes } \\
\hline \multicolumn{4}{|l|}{ Rheidae } \\
\hline Rhea americana (Linnaeus, 1758) NT & Greater Rhea & 4,5 & $\mathrm{BD}$ \\
\hline \multicolumn{4}{|l|}{ Tinamiformes } \\
\hline \multicolumn{4}{|l|}{ Tinamidae } \\
\hline Tinamus tao Temminck, $1815 \mathrm{VU}, \mathrm{VU}^{*}$ & Gray Tinamou & $1,2,4,5$ & $\mathrm{BD}$ \\
\hline Crypturellus soui (Hermann, 1783) & Little Tinamou & $1,2,4,5$ & BD \\
\hline Crypturellus undulatus (Temminck, 1815) & Undulated Tinamou & $1,2,4,5$ & BD \\
\hline Crypturellus parvirostris (Wagler, 1827) & Small-billed Tinamou & $1,2,3,4,5$ & BD \\
\hline Crypturellus tataupa (Temminck, 1815) & Tataupa Tinamou & $1,2,4,5$ & BD \\
\hline Rhynchotus rufescens (Temminck, 1815) & Red-winged Tinamou & $2,4,5$ & $\mathrm{BD}$ \\
\hline \multicolumn{4}{|l|}{ Anseriformes } \\
\hline \multicolumn{4}{|l|}{ Anhimidae } \\
\hline [Chauna torquata $($ Oken, 1816)] & Southern Screamer & 4 & 10 \\
\hline \multicolumn{4}{|l|}{ Anatidae } \\
\hline [Dendrocygna viduata (Linnaeus, 1766)] & White-faced Whistling-Duck & 4 & 10 \\
\hline [Dendrocygna autumnalis (Linnaeus, 1758)] & Black-bellied Whistling-Duck & 4 & 10 \\
\hline [Cairina moschata (Linnaeus, 1758)] & Muscovy Duck & 4 & 10 \\
\hline [Amazonetta brasiliensis (Gmelin, 1789) & Brazilian Teal & 4 & 10 \\
\hline \multicolumn{4}{|l|}{ Galliformes } \\
\hline \multicolumn{4}{|l|}{ Cracidae } \\
\hline Penelope superciliaris Temminck, 1815 & Rusty-margined Guan & $1,2,4,5$ & BD \\
\hline [Penelope ochrogaster Pelzeln, 1870] CE, VU, VU* & Chestnut-bellied Guan & 4 & 10 \\
\hline Aburria cujubi (Pelzeln, 1858) & Red-throated Piping-Guan & 4,5 & $\mathrm{BD}$ \\
\hline [Ortalis canicollis (Wagler, 1830)] & Chaco Chachalaca & 4,5 & 10 \\
\hline Crax fasciolata Spix, $1825 \mathrm{VU}$ & Bare-faced Curassow & 4,5 & $\mathrm{BD}$ \\
\hline \multicolumn{4}{|l|}{ Odontophoridae } \\
\hline Odontophorus gujanensis (Gmelin, 1789) NT & Marbled Wood-Quail & $1,2,4,5$ & $\mathrm{BD}$ \\
\hline \multicolumn{4}{|l|}{ Podicipediformes } \\
\hline \multicolumn{4}{|l|}{ Podicipedidae } \\
\hline Tachybaptus dominicus (Linnaeus, 1766) & Least Grebe & 4,5 & BD \\
\hline [Podilymbus podiceps (Linnaeus, 1758)] & Pied-billed Grebe & 4 & 10 \\
\hline \multicolumn{4}{|l|}{ Ciconiiformes } \\
\hline \multicolumn{4}{|l|}{ Ciconiidae } \\
\hline [Jabiru mycteria (Lichtenstein, 1819)] & Jabiru & 4,5 & 10 \\
\hline Mycteria americana Linnaeus, 1758 & Wood Stork & 4,5 & $\mathrm{BD}$ \\
\hline \multicolumn{4}{|l|}{ Suliformes } \\
\hline \multicolumn{4}{|l|}{ Phalacrocoracidae } \\
\hline [Nannopterum brasilianus (Gmelin, 1789)] & Neotropic Cormorant & 4 & 10 \\
\hline Anhingidae & & & \\
\hline [Anhinga anhinga (Linnaeus, 1766)] & Anhinga & $2,3,4$ & 10 \\
\hline Pelecaniformes & & & \\
\hline Ardeidae & & & \\
\hline [Tigrisoma lineatum (Boddaert, 1783)] & Rufescent Tiger-Heron & 4,5 & 10 \\
\hline [Tigrisoma fasciatum (Such, 1825)] VU* & Fasciated Tiger-Heron & 4 & 10 \\
\hline Agamia agami (Gmelin, 1789) VU & Agami Heron & 5 & $\mathrm{BD}$ \\
\hline [Cochlearius cochlearius (Linnaeus, 1766)] & Boat-billed Heron & 4 & 10 \\
\hline [Nycticorax nycticorax (Linnaeus, 1758)] & Black-crowned Night-Heron & 4 & 10 \\
\hline [Butorides striata (Linnaeus, 1758)] & Striated Heron & 4 & 10 \\
\hline [Bubulcus ibis (Linnaeus, 1758)] & Cattle Egret & 4,5 & 10 \\
\hline [Ardea cocoi Linnaeus, 1766] & Cocoi Heron & 4 & 10 \\
\hline [Ardea alba Linnaeus, 1758] & Great Egret & 4 & 10 \\
\hline [Syrigma sibilatrix (Temminck, 1824)] & Whistling Heron & 4 & 10 \\
\hline [Pilherodius pileatus (Boddaert, 1783)] & Capped Heron & 1,4 & 10 \\
\hline [Egretta thula (Molina, 1782)] & Snowy Egret & 4 & 10 \\
\hline Threskiornithidae & & & \\
\hline [Mesembrinibis cayennensis (Gmelin, 1789)] & Green Ibis & 4,5 & 10 \\
\hline [Phimosus infuscatus (Lichtenstein, 1823)] & Bare-faced lbis & 4 & 10 \\
\hline Theristicus caudatus (Boddaert, 1783) & Buff-necked Ibis & 4,5 & $\mathrm{BD}$ \\
\hline Cathartiformes & & & \\
\hline Cathartidae & & & \\
\hline
\end{tabular}




\begin{tabular}{|c|c|c|c|}
\hline Taxon & English name & Source & Record type \\
\hline Cathartes aura (Linnaeus, 1758) & Turkey Vulture & $1,2,4,5$ & BD \\
\hline [Cathartes burrovianus Cassin, 1845] & Lesser Yellow-headed Vulture & 4,5 & 10 \\
\hline [Cathartes melambrotus Wetmore, 1964] & Greater Yellow-headed Vulture & 5 & 10 \\
\hline Coragyps atratus (Bechstein, 1793) & Black Vulture & $1,2,4,5$ & BD \\
\hline Sarcoramphus papa (Linnaeus, 1758) & King Vulture & $1,2,4,5$ & BD \\
\hline \multicolumn{4}{|l|}{ Accipitriformes } \\
\hline \multicolumn{4}{|l|}{ Accipitridae } \\
\hline [Leptodon cayanensis (Latham, 1790)] & Gray-headed Kite & 4,5 & 10 \\
\hline Chondrohierax uncinatus (Temminck, 1822) & Hook-billed Kite & 4,5 & BD \\
\hline [Elanoides forficatus (Linnaeus, 1758)] & Swallow-tailed Kite & 1,4 & 10 \\
\hline [Gampsonyx swainsonii Vigors, 1825] & Pearl Kite & 1,4 & 10 \\
\hline [Elanus leucurus (Vieillot, 1818)] & White-tailed Kite & 4 & 10 \\
\hline Harpagus bidentatus (Latham, 1790) & Double-toothed Kite & 5 & BD \\
\hline Harpagus diodon (Temminck, 1823) & Rufous-thighed Kite & 5 & BD \\
\hline Accipiter superciliosus (Linnaeus, 1766) & Tiny Hawk & 5 & BD \\
\hline [Accipiter striatus Vieillot, 1808] & Sharp-shinned Hawk & 4 & 10 \\
\hline [Accipiter bicolor (Vieillot, 1817)] & Bicolored Hawk & 4,5 & 10 \\
\hline [/ctinia mississippiensis (Wilson, 1811)] & Mississippi Kite & 4 & 10 \\
\hline Ictinia plumbea (Gmelin, 1788) & Plumbeous Kite & 4,5 & $\mathrm{BD}$ \\
\hline [Busarellus nigricollis (Latham, 1790)] & Black-collared Hawk & 4 & 10 \\
\hline [Rostrhamus sociabilis (Vieillot, 1817)] & Snail Kite & 4 & 10 \\
\hline [Geranospiza caerulescens (Vieillot, 1817)] & Crane Hawk & 1,4 & 10 \\
\hline [Heterospizias meridionalis (Latham, 1790)] & Savanna Hawk & 4 & 10 \\
\hline Urubitinga urubitinga (Gmelin, 1788) & Great Black Hawk & 4,5 & BD \\
\hline [Urubitinga coronata (Vieillot, 1817)] EN, EN* & Crowned Eagle & 4 & 10 \\
\hline Rupornis magnirostris (Gmelin, 1788) & Roadside Hawk & $1,2,4,5$ & $A D$ \\
\hline [Parabuteo unicinctus (Temminck, 1824)] & Harris's Hawk & 4 & 10 \\
\hline Geranoaetus albicaudatus (Vieillot, 1816) & White-tailed Hawk & $2,4,5$ & BD \\
\hline [Geranoaetus melanoleucus (Vieillot, 1819)] & Black-chested Buzzard-Eagle & 4 & 10 \\
\hline Pseudastur albicollis (Latham, 1790) & White Hawk & $2,4,5$ & $\mathrm{BD}$ \\
\hline Buteo nitidus (Latham, 1790) & Gray-lined Hawk & $2,4,5$ & $\mathrm{BD}$ \\
\hline [Buteo brachyurus Vieillot, 1816] & Short-tailed Hawk & 4 & 10 \\
\hline [Buteo albonotatus Kaup, 1847] & Zone-tailed Hawk & 4 & 10 \\
\hline [Harpia harpyja (Linnaeus, 1758)] NT, VU* & Harpy Eagle & 4 & 10 \\
\hline Spizaetus tyrannus (Wied, 1820) & Black Hawk-Eagle & 4,5 & BD \\
\hline [Spizaetus melanoleucus (Vieillot, 1816)] & Black-and-white Hawk-Eagle & 2,4 & 10 \\
\hline Spizaetus ornatus (Daudin, 1800) NT & Ornate Hawk-Eagle & $2,4,5$ & BD \\
\hline \multicolumn{4}{|l|}{ Eurypygiformes } \\
\hline \multicolumn{4}{|l|}{ Eurypygidae } \\
\hline Eurypyga helias (Pallas, 1781) & Sunbittern & $1,4,5$ & BD \\
\hline \multicolumn{4}{|l|}{ Gruiformes } \\
\hline \multicolumn{4}{|l|}{ Aramidae } \\
\hline [Aramus guarauna (Linnaeus, 1766)] & Limpkin & 4 & 10 \\
\hline \multicolumn{4}{|l|}{ Rallidae } \\
\hline [Micropygia schomburgkii (Schomburgk, 1848)] & Ocellated Crake & $1,2,4$ & 10 \\
\hline [Aramides cajaneus (Statius Müller, 1776)] & Gray-necked Wood-Rail & $1,4,5$ & 10 \\
\hline Laterallus viridis (Statius Müller, 1776) & Russet-crowned Crake & $1,2,4$ & $A D$ \\
\hline [Laterallus melanophaius (Vieillot, 1819)] & Rufous-sided Crake & 1,4 & 10 \\
\hline Mustelirallus albicollis (Vieillot, 1819) & Ash-throated Crake & 4,5 & $\mathrm{BD}$ \\
\hline [Porphyrio martinicus (Linnaeus, 1766)] & Purple Gallinule & 4 & 10 \\
\hline \multicolumn{4}{|l|}{ Heliornithidae } \\
\hline [Heliornis fulica (Boddaert, 1783)] & Sungrebe & $\mathrm{mp}$ & 10 \\
\hline \multicolumn{4}{|l|}{ Charadriiformes } \\
\hline \multicolumn{4}{|l|}{ Charadriidae } \\
\hline [Vanellus cayanus (Latham, 1790)] & Pied Lapwing & 4 & 10 \\
\hline Vanellus chilensis (Molina, 1782) & Southern Lapwing & 4,5 & $\mathrm{BD}$ \\
\hline \multicolumn{4}{|l|}{ Scolopacidae } \\
\hline [Gallinago paraguaiae (Vieillot, 1816)] & South American Snipe & 4 & 10 \\
\hline [Gallinago undulata (Boddaert, 1783)] & Giant Snipe & 4 & 10 \\
\hline [Tringa solitaria Wilson, 1813] & Solitary Sandpiper & 4 & 10 \\
\hline \multicolumn{4}{|l|}{ Jacanidae } \\
\hline Jacana jacana (Linnaeus, 1766) & Wattled Jacana & 4,5 & $\mathrm{BD}$ \\
\hline \multicolumn{4}{|l|}{ Columbiformes } \\
\hline Columbidae & & & \\
\hline [Columbina minuta (Linnaeus, 1766)] & Plain-breasted Ground-Dove & 1,4 & 10 \\
\hline Columbina talpacoti (Temminck, 1810) & Ruddy Ground-Dove & $1,2,3,4,5$ & $\mathrm{BD}$ \\
\hline Columbina squammata (Lesson, 1831) & Scaled Dove & $1,4,5$ & $\mathrm{BD}$ \\
\hline [Columbina cyanopis (Pelzeln, 1870)] CE, CR, CR* & Blue-eyed Ground-Dove & 1,4 & 10 \\
\hline
\end{tabular}




\begin{tabular}{|c|c|c|c|}
\hline Taxon & English name & Source & Record type \\
\hline Claravis pretiosa (Ferrari-Perez, 1886) & Blue Ground-Dove & $1,2,3,4,5$ & $A D$ \\
\hline [Uropelia campestris (Spix, 1825)] & Long-tailed Ground-Dove & 1,4 & 10 \\
\hline Patagioenas speciosa (Gmelin, 1789) & Scaled Pigeon & $1,2,3,4,5$ & $\mathrm{BD}$ \\
\hline [Patagioenas picazuro (Temminck, 1813)] & Picazuro Pigeon & $2,4,5$ & 10 \\
\hline Patagioenas cayennensis (Bonnaterre, 1792) & Pale-vented Pigeon & $1,4,5$ & $\mathrm{BD}$ \\
\hline Patagioenas plumbea (Vieillot, 1818) & Plumbeous Pigeon & $2,4,5$ & $\mathrm{BD}$ \\
\hline [Zenaida auriculata (Des Murs, 1847)] & Eared Dove & 4 & 10 \\
\hline Leptotila verreauxi Bonaparte, 1855 & White-tipped Dove & $1,2,3,4,5$ & $A D$ \\
\hline Leptotila rufaxilla (Richard \& Bernard, 1792) & Gray-fronted Dove & $1,2,4,5$ & $\mathrm{BD}$ \\
\hline Geotrygon montana (Linnaeus, 1758) & Ruddy Quail-Dove & $1,3,4,5$ & $\mathrm{BD}$ \\
\hline \multicolumn{4}{|l|}{ Cuculiformes } \\
\hline \multicolumn{4}{|l|}{ Cuculidae } \\
\hline [Coccycua minuta (Vieillot, 1817)] & Little Cuckoo & 4 & 10 \\
\hline Piaya cayana (Linnaeus, 1766) & Squirrel Cuckoo & $1,2,4,5$ & $\mathrm{BD}$ \\
\hline Coccyzus euleri Cabanis, 1873 & Pearly-breasted Cuckoo & 5 & $\mathrm{BD}$ \\
\hline [Crotophaga major Gmelin, 1788] & Greater Ani & 4 & 10 \\
\hline Crotophaga ani Linnaeus, 1758 & Smooth-billed Ani & $1,2,3,4,5$ & $A D$ \\
\hline Guira guira (Gmelin, 1788) & Guira Cuckoo & $1,4,5$ & $\mathrm{BD}$ \\
\hline Tapera naevia (Linnaeus, 1766) & Striped Cuckoo & $1,3,4$ & $A D$ \\
\hline [Dromococcyx phasianellus (Spix, 1824)] & Pheasant Cuckoo & 2,4 & 10 \\
\hline Dromococcyx pavoninus Pelzeln, 1870 & Pavonine Cuckoo & $2,4,5$ & $\mathrm{BD}$ \\
\hline \multicolumn{4}{|l|}{ Strigiformes } \\
\hline \multicolumn{4}{|l|}{ Tytonidae } \\
\hline [Tyto furcata (Temminck, 1827)] & American Barn Owl & 4 & 10 \\
\hline \multicolumn{4}{|l|}{ Strigidae Leach, 1820} \\
\hline Megascops choliba (Vieillot, 1817) & Tropical Screech-Owl & $1,4,5$ & $\mathrm{BD}$ \\
\hline Megascops usta (Sclater, 1858) & Austral Screech-Owl & 4,5 & $\mathrm{BD}$ \\
\hline Pulsatrix perspicillata (Latham, 1790) & Spectacled Owl & $2,4,5$ & $\mathrm{BD}$ \\
\hline [Bubo virginianus (Gmelin, 1788)] & Great Horned Owl & 4 & 10 \\
\hline [Strix virgata (Cassin, 1849)] & Mottled Owl & 4,5 & 10 \\
\hline [Strix huhula Daudin, 1800] & Black-banded Owl & 4 & 10 \\
\hline Glaucidium brasilianum (Gmelin, 1788) & Ferruginous Pygmy-Owl & $2,4,5$ & $\mathrm{BD}$ \\
\hline Athene cunicularia (Molina, 1782) & Burrowing Owl & $1,4,5$ & $\mathrm{BD}$ \\
\hline Asio clamator (Vieillot, 1808) & Striped Owl & 4,5 & $\mathrm{BD}$ \\
\hline [Asio stygius (Wagler, 1832)] & Stygian Owl & 4 & 10 \\
\hline \multicolumn{4}{|l|}{ Nyctibiiformes } \\
\hline \multicolumn{4}{|l|}{ Nyctibiidae } \\
\hline [Nyctibius grandis (Gmelin, 1789)] & Great Potoo & 4 & 10 \\
\hline Nyctibius griseus (Gmelin, 1789) & Common Potoo & 4,5 & $\mathrm{BD}$ \\
\hline \multicolumn{4}{|l|}{ Caprimulgiformes } \\
\hline \multicolumn{4}{|l|}{ Caprimulgidae } \\
\hline Antrostomus rufus (Boddaert, 1783) & Rufous Nightjar & $3,4,5$ & $\mathrm{BD}$ \\
\hline Lurocalis semitorquatus (Gmelin, 1789) & Short-tailed Nighthawk & 5 & $\mathrm{BD}$ \\
\hline Nyctidromus albicollis (Gmelin, 1789) & Common Pauraque & $1,2,3,4,5$ & $\mathrm{BD}$ \\
\hline Hydropsalis parvula (Gould, 1837) & Little Nightjar & 4,5 & $\mathrm{BD}$ \\
\hline Hydropsalis longirostris (Bonaparte, 1825) & Band-winged Nightjar & 4,5 & $\mathrm{BD}$ \\
\hline Hydropsalis torquata (Gmelin, 1789) & Scissor-tailed Nightjar & $1,2,4,5$ & $A D$ \\
\hline [Nannochordeiles pusillus (Gould, 1861)] & Least Nighthawk & 4 & 10 \\
\hline [Podager nacunda (Vieillot, 1817)] & Nacunda Nighthawk & 4 & 10 \\
\hline [Chordeiles minor (Forster, 1771)] & Common Nighthawk & 4 & 10 \\
\hline [Chordeiles acutipennis (Hermann, 1783)] & Lesser Nighthawk & 1,4 & 10 \\
\hline \multicolumn{4}{|l|}{ Apodiformes } \\
\hline \multicolumn{4}{|l|}{ Apodidae } \\
\hline [Cypseloides fumigatus (Streubel, 1848)] & Sooty Swift & 2,4 & 10 \\
\hline Cypseloides senex (Temminck, 1826) & Great Dusky Swift & 5 & $\mathrm{BD}$ \\
\hline Streptoprocne zonaris (Shaw, 1796) & White-collared Swift & $2,4,5$ & $\mathrm{BD}$ \\
\hline [Streptoprocne biscutata (Sclater, 1866)] & Biscutate Swift & 4 & 10 \\
\hline [Chaetura meridionalis Hellmayr, 1907] & Sick's Swift & 4 & 10 \\
\hline [Tachornis squamata (Cassin, 1853)] & Fork-tailed Palm-Swift & 1,4 & 10 \\
\hline \multicolumn{4}{|l|}{ Trochilidae } \\
\hline Glaucis hirsutus (Gmelin, 1788) & Rufous-breasted Hermit & $1,3,4,5$ & $A D$ \\
\hline Phaethornis nattereri Berlepsch, 1887 & Cinnamon-throated Hermit & $1,4,5$ & $A D$ \\
\hline [Phaethornis ruber (Linnaeus, 1758)] & Reddish Hermit & 4,5 & 10 \\
\hline [Phaethornis subochraceus Todd, 1915] & Buff-bellied Hermit & 4 & 10 \\
\hline Phaethornis pretrei (Lesson \& Delattre, 1839) & Planalto Hermit & $1,2,3,4,5$ & $\mathrm{BD}$ \\
\hline Eupetomena macroura (Gmelin, 1788) & Swallow-tailed Hummingbird & $1,2,3,4,5$ & $A D$ \\
\hline Colibri serrirostris (Vieillot, 1816) & White-vented Violetear & $3,4,5$ & $A D$ \\
\hline Anthracothorax nigricollis (Vieillot, 1817) & Black-throated Mango & $1,2,4,5$ & $A D$ \\
\hline
\end{tabular}




\begin{tabular}{|c|c|c|c|}
\hline Taxon & English name & Source & Record type \\
\hline [Chrysolampis mosquitus (Linnaeus, 1758)] & Ruby-topaz Hummingbird & 4 & 10 \\
\hline Lophornis gouldii (Lesson, 1832) VU, VU* & Dot-eared Coquette & 5 & BD \\
\hline Chlorostilbon lucidus (Shaw, 1812) & Glittering-bellied Emerald & $3,2,4,5$ & BD \\
\hline Thalurania furcata (Gmelin, 1788) & Fork-tailed Woodnymph & $1,2,3,4,5$ & $A D$ \\
\hline Hylocharis cyanus (Vieillot, 1818) & White-chinned Sapphire & 5 & BD \\
\hline Hylocharis chrysura (Shaw, 1812) & Gilded Hummingbird & 4,5 & $A D$ \\
\hline [Polytmus guainumbi (Pallas, 1764)] & White-tailed Goldenthroat & 4 & 10 \\
\hline Amazilia fimbriata (Gmelin, 1788) & Glittering-throated Emerald & $1,2,4,5$ & BD \\
\hline [Heliactin bilophus (Temminck, 1820)] & Horned Sungem & $1,2,4$ & 10 \\
\hline Heliomaster longirostris (Audebert \& Vieillot, 1801) & Long-billed Starthroat & $2,4,5$ & BD \\
\hline [Heliomaster furcifer (Shaw, 1812)] & Blue-tufted Starthroat & 4,5 & 10 \\
\hline Calliphlox amethystina (Boddaert, 1783) & Amethyst Woodstar & 4,5 & BD \\
\hline \multicolumn{4}{|l|}{ Trogoniformes } \\
\hline \multicolumn{4}{|l|}{ Trogonidae } \\
\hline Trogon melanurus Swainson, 1838 & Black-tailed Trogon & 4,5 & BD \\
\hline Trogon viridis Linnaeus, 1766 & Green-backed Trogon & $1,4,5$ & BD \\
\hline Trogon curucui Linnaeus, 1766 & Blue-crowned Trogon & $1,2,3,4,5$ & $A D$ \\
\hline Trogon collaris Vieillot, 1817 & Collared Trogon & 4,5 & $\mathrm{BD}$ \\
\hline \multicolumn{4}{|l|}{ Coraciiformes } \\
\hline \multicolumn{4}{|l|}{ Alcedinidae } \\
\hline Megaceryle torquata (Linnaeus, 1766) & Ringed Kingfisher & $1,4,5$ & BD \\
\hline Chloroceryle amazona (Latham, 1790) & Amazon Kingfisher & $1,2,4,5$ & BD \\
\hline [Chloroceryle aenea (Pallas, 1764)] & American Pygmy Kingfisher & $1,4,5$ & 10 \\
\hline Chloroceryle americana (Gmelin, 1788) & Green Kingfisher & $1,4,5$ & $\mathrm{BD}$ \\
\hline Chloroceryle inda (Linnaeus, 1766) & Green-and-rufous Kingfisher & $2,4,5$ & $\mathrm{BD}$ \\
\hline \multicolumn{4}{|l|}{ Momotidae } \\
\hline [Electron platyrhynchum (Leadbeater, 1829)] & Broad-billed Motmot & 1,4 & 10 \\
\hline Momotus momota (Linnaeus, 1766) & Amazonian Motmot & $1,2,3,4,5$ & $A D$ \\
\hline \multicolumn{4}{|l|}{ Galbuliformes } \\
\hline \multicolumn{4}{|l|}{ Galbulidae } \\
\hline Brachygalba lugubris (Swainson, 1838) & Brown Jacamar & $1,2,4$ & $A D$ \\
\hline Galbula ruficauda Cuvier, 1816 & Rufous-tailed Jacamar & $1,2,4,5$ & BD \\
\hline \multicolumn{4}{|l|}{ Bucconidae } \\
\hline [Notharchus hyperrhynchus (Sclater, 1856)] & White-necked Puffbird & 1 & 10 \\
\hline Notharchus tectus (Boddaert, 1783) & Pied Puffbird & $2,4,5$ & $\mathrm{BD}$ \\
\hline Bucco tamatia Gmelin, 1788 & Spotted Puffbird & 4,5 & BD \\
\hline Nystalus striolatus (Pelzeln, 1856) & Natterer's Striolated Puffbird & 4,5 & $\mathrm{BD}$ \\
\hline Nystalus chacuru (Vieillot, 1816) & White-eared Puffbird & $1,2,3,4,5$ & $A D$ \\
\hline Nystalus maculatus (Gmelin, 1788) & Spot-backed Puffbird & 4,5 & $\mathrm{BD}$ \\
\hline Nonnula ruficapilla (Tschudi, 1844) & Rufous-capped Nunlet & 4,5 & BD \\
\hline Monasa nigrifrons (Spix, 1824) & Black-fronted Nunbird & $1,2,3,4,5$ & $A D$ \\
\hline [Monasa morphoeus (Hahn \& Küster, 1823)] & White-fronted Nunbird & 1 & 10 \\
\hline Chelidoptera tenebrosa (Pallas, 1782) & Swallow-winged Puffbird & $2,4,5$ & $\mathrm{BD}$ \\
\hline \multicolumn{4}{|l|}{ Piciformes } \\
\hline \multicolumn{4}{|l|}{ Ramphastidae } \\
\hline Ramphastos toco Statius Müller, 1776 & Toco Toucan & $1,4,5$ & BD \\
\hline Ramphastos tucanus Linnaeus, 1758 & White-throated Toucan & 5 & BD \\
\hline [Ramphastos vitellinus Lichtenstein, 1823] & Channel-billed Toucan & 1,4 & 10 \\
\hline Pteroglossus inscriptus Swainson, 1822 & Lettered Aracari & $1,2,3,4,5$ & $\mathrm{BD}$ \\
\hline [Pteroglossus bitorquatus Vigors, 1826] EN & Red-necked Aracari & 1,4 & 10 \\
\hline Pteroglossus castanotis Gould, 1834 & Chestnut-eared Aracari & $1,2,4,5$ & $A D$ \\
\hline \multicolumn{4}{|l|}{ Picidae } \\
\hline [Picumnus albosquamatus d'Orbigny, 1840] & White-wedged Piculet & $1,4,5$ & 10 \\
\hline Melanerpes candidus (Otto, 1796) & White Woodpecker & 4,5 & BD \\
\hline Melanerpes cruentatus (Boddaert, 1783) & Yellow-tufted Woodpecker & $1,2,3,4,5$ & $A D$ \\
\hline Veniliornis affinis (Swainson, 1821) & Red-stained Woodpecker & 5 & BD \\
\hline Veniliornis passerinus (Linnaeus, 1766) & Little Woodpecker & $2,3,4,5$ & $A D$ \\
\hline [Veniliornis mixtus (Boddaert, 1783)] & Checkered Woodpecker & 2,4 & 10 \\
\hline [Piculus leucolaemus (Natterer \& Malherbe, 1845)] & White-throated Woodpecker & 4 & 10 \\
\hline [Piculus chrysochloros (Vieillot, 1818)] & Golden-green Woodpecker & $2,4,5$ & 10 \\
\hline Colaptes melanochloros (Gmelin, 1788) & Green-barred Woodpecker & $2,4,5$ & BD \\
\hline [Colaptes campestris (Vieillot, 1818)] & Campo Flicker & $1,4,5$ & 10 \\
\hline Celeus torquatus (Boddaert, 1783) & Ringed Woodpecker & 5 & $\mathrm{BD}$ \\
\hline [Celeus elegans (Statius Müller, 1776)] & Chestnut Woodpecker & 1,4 & 10 \\
\hline Celeus lugubris (Malherbe, 1851) & Pale-crested Woodpecker & $1,2,3,4,5$ & BD \\
\hline Dryocopus lineatus (Linnaeus, 1766) & Lineated Woodpecker & $1,4,5$ & BD \\
\hline Campephilus rubricollis (Boddaert, 1783) & Red-necked Woodpecker & $2,4,5$ & BD \\
\hline [Campephilus melanoleucos (Gmelin, 1788)] & Crimson-crested Woodpecker & $1,4,5$ & 10 \\
\hline
\end{tabular}




\begin{tabular}{|c|c|c|c|}
\hline Taxon & English name & Source & Record type \\
\hline \multicolumn{4}{|l|}{ Cariamiformes } \\
\hline \multicolumn{4}{|l|}{ Cariamidae } \\
\hline Cariama cristata (Linnaeus, 1766) & Red-legged Seriema & $1,4,5$ & $\mathrm{BD}$ \\
\hline \multicolumn{4}{|l|}{ Falconiformes } \\
\hline \multicolumn{4}{|l|}{ Falconidae } \\
\hline [Daptrius ater Vieillot, 1816] & Black Caracara & 4 & 10 \\
\hline [Caracara plancus (Miller, 1777)] & Southern Caracara & $1,4,5$ & 10 \\
\hline [Milvago chimachima (Vieillot, 1816)] & Yellow-headed Caracara & 1,4 & 10 \\
\hline Herpetotheres cachinnans (Linnaeus, 1758) & Laughing Falcon & $1,2,4,5$ & $\mathrm{BD}$ \\
\hline Micrastur ruficollis (Vieillot, 1817) & Barred Forest-Falcon & $2,4,5$ & $\mathrm{BD}$ \\
\hline Micrastur semitorquatus (Vieillot, 1817) & Collared Forest-Falcon & $1,4,5$ & $\mathrm{BD}$ \\
\hline [Falco sparverius Linnaeus, 1758] & American Kestrel & $2,4,5$ & 10 \\
\hline Falco rufigularis Daudin, 1800 & Bat Falcon & $1,2,4,5$ & $\mathrm{BD}$ \\
\hline [Falco femoralis Temminck, 1822] & Aplomado Falcon & 4 & 10 \\
\hline \multicolumn{4}{|l|}{ Psittaciformes } \\
\hline \multicolumn{4}{|l|}{ Psittacidae } \\
\hline [Anodorhynchus hyacinthinus (Latham, 1790)] VU & Hyacinth Macaw & $1,4,5$ & 10 \\
\hline Ara ararauna (Linnaeus, 1758) & Blue-and-yellow Macaw & $1,2,4,5$ & $\mathrm{BD}$ \\
\hline Ara chloropterus Gray, 1859 & Red-and-green Macaw & $1,2,4,5$ & $\mathrm{BD}$ \\
\hline [Orthopsittaca manilatus (Boddaert, 1783)] & Red-bellied Macaw & $1,4,5$ & 10 \\
\hline [Primolius maracana (Vieillot, 1816)] NT & Blue-winged Macaw & 4 & 10 \\
\hline [Primolius auricollis (Cassin, 1853)] & Yellow-collared Macaw & 5 & 10 \\
\hline Diopsittaca nobilis (Linnaeus, 1758) & Red-shouldered Macaw & $1,2,4,5$ & $\mathrm{BD}$ \\
\hline [Psittacara leucophthalmus (Statius Müller, 1776)] & White-eyed Parakeet & $1,4,5$ & 10 \\
\hline Aratinga weddellii (Deville, 1851) & Dusky-headed Parakeet & $2,4,5$ & $\mathrm{BD}$ \\
\hline Eupsittula aurea (Gmelin, 1788) & Peach-fronted Parakeet & $1,2,4,5$ & $\mathrm{BD}$ \\
\hline [Forpus xanthopterygius (Spix, 1824)] & Blue-winged Parrotlet & 4 & 10 \\
\hline Brotogeris chiriri (Vieillot, 1818) & Yellow-chevroned Parakeet & $1,2,3,4,5$ & $A D$ \\
\hline [Alipiopsitta xanthops (Spix, 1824)] CE, NT & Yellow-faced Parrot & 4 & 10 \\
\hline Pionus menstruus (Linnaeus, 1766) & Blue-headed Parrot & $1,2,4,5$ & $\mathrm{BD}$ \\
\hline Amazona amazonica (Linnaeus, 1766) & Orange-winged Parrot & 4,5 & $\mathrm{BD}$ \\
\hline [Amazona aestiva (Linnaeus, 1758)] & Turquoise-fronted Parrot & 4,5 & 10 \\
\hline \multicolumn{4}{|l|}{ Passeriformes } \\
\hline \multicolumn{4}{|l|}{ Thamnophilidae } \\
\hline [Microrhopias quixensis (Cornalia, 1849)] & Dot-winged Antwren & 2,4 & 10 \\
\hline Myrmophylax atrothorax (Boddaert, 1783) & Black-throated Antbird & $1,2,4,5$ & $A D$ \\
\hline Formicivora grisea (Boddaert, 1783) & White-fringed Antwren & $1,2,3,4,5$ & $A D$ \\
\hline Formicivora rufa (Wied, 1831) & Rusty-backed Antwren & $1,2,3,4,5$ & $A D$ \\
\hline [Isleria hauxwelli (Sclater, 1857)] & Plain-throated Antwren & 1,4 & 10 \\
\hline Dysithamnus mentalis (Temminck, 1823) & Plain Antvireo & $1,2,4,5$ & $\mathrm{BD}$ \\
\hline Herpsilochmus longirostris Pelzeln, 1868 CE & Large-billed Antwren & 4,5 & $\mathrm{BD}$ \\
\hline Thamnophilus doliatus (Linnaeus, 1764) & Barred Antshrike & $1,2,4,5$ & $\mathrm{BD}$ \\
\hline [Thamnophilus torquatus Swainson, 1825] & Rufous-winged Antshrike & 1,4 & 10 \\
\hline Thamnophilus schistaceus d'Orbigny, 1835 & Plain-winged Antshrike & 5 & $\mathrm{BD}$ \\
\hline [Thamnophilus stictocephalus Pelzeln, 1868] & Natterer's Slaty-Antshrike & 1,2 & 10 \\
\hline Thamnophilus pelzelni Hellmayr, 1924 & Planalto Slaty-Antshrike & 4,5 & $A D$ \\
\hline [Thamnophilus caerulescens Vieillot, 1816] & Variable Antshrike & 1 & 10 \\
\hline Thamnophilus aethiops Sclater, 1858 & White-shouldered Antshrike & 5 & $\mathrm{BD}$ \\
\hline Thamnophilus amazonicus Sclater, 1858 & Amazonian Antshrike & 5 & $A D$ \\
\hline [Cymbilaimus lineatus (Leach, 1814)] & Fasciated Antshrike & 1 & 10 \\
\hline [Taraba major (Vieillot, 1816)] & Great Antshrike & $1,2,3,4,5$ & 10 \\
\hline [Hypocnemoides maculicauda (Pelzeln, 1868)] & Band-tailed Antbird & 2,4 & 10 \\
\hline [Myrmoborus myotherinus (Spix, 1825)] & Black-faced Antbird & 1,4 & 10 \\
\hline Pyriglena leuconota (Spix, 1824) & White-backed Fire-eye & $1,2,3,4,5$ & $A D$ \\
\hline [Cercomacroides nigrescens (Cabanis \& Heine, 1859)] & Blackish Antbird & 1,4 & 10 \\
\hline Hypocnemis ochrogyna Zimmer, 1932 VU* & Rondonia Warbling-Antbird & $2,4,5$ & $\mathrm{BD}$ \\
\hline Willisornis poecilinotus (Cabanis, 1847) & Common Scale-backed Antbird & $1,4,5$ & $A D$ \\
\hline [Rhegmatorhina hoffmannsi (Hellmayr, 1907)] NT & White-breasted Antbird & 1,4 & 10 \\
\hline \multicolumn{4}{|l|}{ Melanopareiidae } \\
\hline Melanopareia torquata (Wied, 1831) CE & Collared Crescentchest & $1,2,4,5$ & $\mathrm{BD}$ \\
\hline \multicolumn{4}{|l|}{ Conopophagidae } \\
\hline [Conopophaga lineata (Wied, 1831)] & Rufous Gnateater & 1,4 & 10 \\
\hline \multicolumn{4}{|l|}{ Dendrocolaptidae } \\
\hline Dendrocincla fuliginosa (Vieillot, 1818) & Plain-brown Woodcreeper & $1,4,5$ & $A D$ \\
\hline Sittasomus griseicapillus (Vieillot, 1818) & Olivaceous Woodcreeper & $1,2,3,4,5$ & $A D$ \\
\hline [Glyphorynchus spirurus (Vieillot, 1819)] & Wedge-billed Woodcreeper & 1,4 & 10 \\
\hline Xiphorhynchus guttatoides (Lafresnaye, 1850) & Lafresnaye's Woodcreeper & $1,2,3,4,5$ & $A D$ \\
\hline [Dendroplex picus (Gmelin, 1788)] & Straight-billed Woodcreeper & $1,4,5$ & 10 \\
\hline
\end{tabular}




\begin{tabular}{|c|c|c|c|}
\hline Taxon & English name & Source & Record type \\
\hline [Lepidocolaptes angustirostris (Vieillot, 1818)] & Narrow-billed Woodcreeper & 1,4 & 10 \\
\hline [Lepidocolaptes fuscicapillus (Pelzeln, 1868)] & Rondonia Woodcreeper & $2,4,5$ & 10 \\
\hline [Dendrocolaptes platyrostris Spix, 1825] & Planalto Woodcreeper & 2,4 & 10 \\
\hline [Hylexetastes uniformis Hellmayr, 1909] & Uniform Woodcreeper & 1,4 & 10 \\
\hline \multicolumn{4}{|l|}{ Xenopidae } \\
\hline Xenops rutilans Temminck, 1821 & Streaked Xenops & $1,4,5$ & $\mathrm{BD}$ \\
\hline \multicolumn{4}{|l|}{ Furnariidae } \\
\hline Furnarius rufus (Gmelin, 1788) & Rufous Hornero & $1,2,3,4,5$ & $A D$ \\
\hline [Lochmias nematura (Lichtenstein, 1823)] & Sharp-tailed Streamcreeper & 4 & 10 \\
\hline [Clibanornis rectirostris (Wied, 1831)] CE & Chestnut-capped Foliage-gleaner & 4 & 10 \\
\hline [Syndactyla dimidiata (Pelzeln, 1859)] & Russet-mantled Foliage-gleaner & 1,4 & 10 \\
\hline [Phacellodomus rufifrons (Wied, 1821)] & Rufous-fronted Thornbird & 4 & 10 \\
\hline [Phacellodomus ruber (Vieillot, 1817)] & Greater Thornbird & 4 & 10 \\
\hline Synallaxis frontalis Pelzeln, 1859 & Sooty-fronted Spinetail & $1,4,5$ & $\mathrm{BD}$ \\
\hline Synallaxis albescens Temminck, 1823 & Pale-breasted Spinetail & $1,2,4$ & $A D$ \\
\hline [Synallaxis rutilans Temminck, 1823] & Ruddy Spinetail & 1,4 & 10 \\
\hline [Synallaxis gujanensis (Gmelin, 1789)] & Plain-crowned Spinetail & 1,4 & 10 \\
\hline [Cranioleuca vulpina (Pelzeln, 1856)] & Rusty-backed Spinetail & 1,4 & 10 \\
\hline \multicolumn{4}{|l|}{ Pipridae Rafinesque, 1815} \\
\hline Neopelma pallescens (Lafresnaye, 1853) & Pale-bellied Tyrant-Manakin & $1,2,4,5$ & $A D$ \\
\hline [Tyranneutes stolzmanni (Hellmayr, 1906)] & Dwarf Tyrant-Manakin & 1,4 & 10 \\
\hline Pipra fasciicauda Hellmayr, 1906 & Band-tailed Manakin & $1,2,3,4,5$ & $A D$ \\
\hline Manacus manacus (Linnaeus, 1766) & White-bearded Manakin & $1,2,3,4,5$ & $A D$ \\
\hline Machaeropterus pyrocephalus (Sclater, 1852) & Fiery-capped Manakin & $1,2,3,4,5$ & $A D$ \\
\hline Chiroxiphia pareola (Linnaeus, 1766) & Blue-backed Manakin & 5 & $A D$ \\
\hline Antilophia galeata (Lichtenstein, 1823) CE & Helmeted Manakin & $1,2,4,5$ & $A D$ \\
\hline \multicolumn{4}{|l|}{ Oxyruncidae } \\
\hline Oxyruncus cristatus Swainson, 1821 & Sharpbill & $2,4,5$ & $\mathrm{BD}$ \\
\hline \multicolumn{4}{|l|}{ Onychorhynchidae } \\
\hline [Terenotriccus erythrurus (Cabanis, 1847)] & Ruddy-tailed Flycatcher & 1 & 10 \\
\hline \multicolumn{4}{|l|}{ Tityridae } \\
\hline [Schiffornis turdina (Wied, 1831)] & Thrush-like Schiffornis & 4 & 10 \\
\hline [Tityra inquisitor (Lichtenstein, 1823)] & Black-crowned Tityra & 4 & 10 \\
\hline [Tityra cayana (Linnaeus, 1766)] & Black-tailed Tityra & $1,4,5$ & 10 \\
\hline Tityra semifasciata (Spix, 1825) & Masked Tityra & $1,2,3,4,5$ & $A D$ \\
\hline [Pachyramphus viridis (Vieillot, 1816)] & Green-backed Becard & 4 & 10 \\
\hline [Pachyramphus polychopterus (Vieillot, 1818)] & White-winged Becard & $1,4,5$ & 10 \\
\hline \multicolumn{4}{|l|}{ Cotingidae Bonaparte, 1849} \\
\hline [Gymnoderus foetidus (Linnaeus, 1758)] & Bare-necked Fruitcrow & 5 & 10 \\
\hline [Xipholena punicea (Pallas, 1764)] & Pompadour Cotinga & 1,4 & 10 \\
\hline \multicolumn{4}{|l|}{ Pipritidae } \\
\hline [Piprites chloris (Temminck, 1822)] & Wing-barred Piprites & 1,4 & 10 \\
\hline \multicolumn{4}{|l|}{ Platyrinchidae } \\
\hline Platyrinchus mystaceus Vieillot, 1818 & White-throated Spadebill & $2,4,5$ & $\mathrm{BD}$ \\
\hline \multicolumn{4}{|l|}{ Rhynchocyclidae } \\
\hline Mionectes oleagineus (Lichtenstein, 1823) & Ochre-bellied Flycatcher & $1,3,4,5$ & $\mathrm{BD}$ \\
\hline Leptopogon amaurocephalus Tschudi, 1846 & Sepia-capped Flycatcher & $2,3,4,5$ & $A D$ \\
\hline Corythopis delalandi (Lesson, 1830) & Southern Antpipit & 4,5 & $A D$ \\
\hline [Tolmomyias sulphurescens (Spix, 1825)] & Yellow-olive Flycatcher & $1,2,3,4,5$ & 10 \\
\hline Tolmomyias assimilis (Pelzeln, 1868) & Yellow-margined Flycatcher & 5 & $\mathrm{BD}$ \\
\hline Tolmomyias flaviventris (Wied, 1831) & Yellow-breasted Flycatcher & 5 & $A D$ \\
\hline [Todirostrum cinereum (Linnaeus, 1766)] & Common Tody-Flycatcher & 1,4 & 10 \\
\hline Poecilotriccus latirostris (Pelzeln, 1868) & Rusty-fronted Tody-Flycatcher & $1,2,3,4$ & $A D$ \\
\hline Myiornis ecaudatus (d'Orbigny \& Lafresnaye, 1837) & Short-tailed Pygmy-Tyrant & 5 & $\mathrm{BD}$ \\
\hline Hemitriccus minor (Snethlage, 1907) & Snethlage's Tody-Tyrant & 5 & $\mathrm{BD}$ \\
\hline Hemitriccus flammulatus Berlepsch, 1901 & Flammulated Pygmy-Tyrant & 4,5 & $A D$ \\
\hline Hemitriccus striaticollis (Lafresnaye, 1853) & Stripe-necked Tody-Tyrant & $1,2,3,4,5$ & $A D$ \\
\hline Hemitriccus margaritaceiventer (d'Orbigny \& Lafresnaye, 1837) & Pearly-vented Tody-tyrant & $1,2,4,5$ & $\mathrm{BD}$ \\
\hline \multicolumn{4}{|l|}{ Tyrannidae } \\
\hline Hirundinea ferruginea (Gmelin, 1788) & Cliff Flycatcher & $2,4,5$ & $\mathrm{BD}$ \\
\hline [Euscarthmus meloryphus Wied, 1831] & Tawny-crowned Pygmy-Tyrant & 2,4 & 10 \\
\hline [Euscarthmus rufomarginatus (Pelzeln, 1868)] CE, NT & Rufous-sided Pygmy-Tyrant & 2,4 & 10 \\
\hline Ornithion inerme Hartlaub, 1853 & White-lored Tyrannulet & 5 & $\mathrm{BD}$ \\
\hline Camptostoma obsoletum (Temminck, 1824) & Southern Beardless-Tyrannulet & $1,4,5$ & $\mathrm{BD}$ \\
\hline Elaenia flavogaster (Thunberg, 1822) & Yellow-bellied Elaenia & $1,2,3,4,5$ & $\mathrm{BD}$ \\
\hline Elaenia chilensis Hellmayr, 1927 & Chilean Elaenia & $3,4,5$ & $A D$ \\
\hline Elaenia parvirostris Pelzeln, 1868 & Small-billed Elaenia & $1,4,5$ & $A D$ \\
\hline [Elaenia cristata Pelzeln, 1868] & Plain-crested Elaenia & $1,4,5$ & 10 \\
\hline
\end{tabular}




\begin{tabular}{|c|c|c|c|}
\hline Taxon & English name & Source & Record type \\
\hline Elaenia chiriquensis Lawrence, 1865 & Lesser Elaenia & $2,3,4,5$ & $A D$ \\
\hline [Suiriri suiriri (Vieillot, 1818)] & Suiriri Flycatcher & 1,4 & 10 \\
\hline [Suiriri affinis (Burmeister, 1856)] NT & Chapada Flycatcher & 4 & 10 \\
\hline Myiopagis gaimardii (d'Orbigny, 1839) & Forest Elaenia & $2,4,5$ & $A D$ \\
\hline Myiopagis caniceps (Swainson, 1835) & Gray Elaenia & $2,4,5$ & $\mathrm{BD}$ \\
\hline [Myiopagis viridicata (Vieillot, 1817)] & Greenish Elaenia & $3,2,4$ & 10 \\
\hline Phaeomyias murina (Spix, 1825) & Mouse-colored Tyrannulet & $1,2,3,4,5$ & $A D$ \\
\hline [Phyllomyias fasciatus (Thunberg, 1822)] & Planalto Tyrannulet & 2,4 & 10 \\
\hline [Culicivora caudacuta (Vieillot, 1818)] VU & Sharp-tailed Tyrant & 1,4 & 10 \\
\hline Attila bolivianus Lafresnaye, 1848 & Dull-capped Attila & $1,2,3,4,5$ & $A D$ \\
\hline Legatus leucophaius (Vieillot, 1818) & Piratic Flycatcher & $1,2,4,5$ & $\mathrm{BD}$ \\
\hline [Ramphotrigon ruficauda (Spix, 1825)] & Rufous-tailed Flatbill & 1,4 & 10 \\
\hline Myiarchus tuberculifer (d'Orbigny \& Lafresnaye, 1837) & Dusky-capped Flycatcher & $2,4,5$ & $\mathrm{BD}$ \\
\hline Myiarchus swainsoni Cabanis \& Heine, 1859 & Swainson's Flycatcher & $1,3,4,5$ & $A D$ \\
\hline Myiarchus ferox (Gmelin, 1789) & Short-crested Flycatcher & $1,2,4,5$ & $\mathrm{BD}$ \\
\hline Myiarchus tyrannulus (Statius Müller, 1776) & Brown-crested Flycatcher & $2,3,4,5$ & $A D$ \\
\hline Sirystes sibilator (Vieillot, 1818) & Sibilant Sirystes & $2,4,5$ & $\mathrm{BD}$ \\
\hline Rhytipterna simplex (Lichtenstein, 1823) & Grayish Mourner & 5 & $\mathrm{BD}$ \\
\hline Casiornis rufus (Vieillot, 1816) & Rufous Casiornis & $2,3,4,5$ & $\mathrm{BD}$ \\
\hline Pitangus sulphuratus (Linnaeus, 1766) & Great Kiskadee & $1,2,4,5$ & $\mathrm{BD}$ \\
\hline [Philohydor lictor (Lichtenstein, 1823)] & Lesser Kiskadee & 1,4 & 10 \\
\hline [Machetornis rixosa (Vieillot, 1819)] & Cattle Tyrant & 1,4 & 10 \\
\hline Myiodynastes maculatus (Statius Müller, 1776) & Streaked Flycatcher & $1,4,5$ & $\mathrm{BD}$ \\
\hline Megarynchus pitangua (Linnaeus, 1766) & Boat-billed Flycatcher & $1,2,3,4,5$ & $\mathrm{BD}$ \\
\hline Myiozetetes cayanensis (Linnaeus, 1766) & Rusty-margined Flycatcher & $1,2,4,5$ & $\mathrm{BD}$ \\
\hline [Tyrannus albogularis Burmeister, 1856] & White-throated Kingbird & 4 & 10 \\
\hline Tyrannus melancholicus Vieillot, 1819 & Tropical Kingbird & $1,4,5$ & $\mathrm{BD}$ \\
\hline Tyrannus savana Daudin, 1802 & Fork-tailed Flycatcher & $1,4,5$ & $\mathrm{BD}$ \\
\hline Griseotyrannus aurantioatrocristatus (d'Orbigny \& Lafresnaye, 1837) & Crowned Slaty Flycatcher & 4,5 & $\mathrm{BD}$ \\
\hline Empidonomus varius (Vieillot, 1818) & Variegated Flycatcher & $2,4,5$ & $\mathrm{BD}$ \\
\hline [Colonia colonus (Vieillot, 1818)] & Long-tailed Tyrant & 1,4 & 10 \\
\hline Myiophobus fasciatus (Statius Müller, 1776) & Bran-colored Flycatcher & $1,2,4,5$ & $\mathrm{BD}$ \\
\hline [Sublegatus modestus (Wied, 1831)] & Southern Scrub-Flycatcher & $2,3,4$ & 10 \\
\hline Pyrocephalus rubinus (Boddaert, 1783) & Vermilion Flycatcher & 4,5 & $\mathrm{BD}$ \\
\hline [Gubernetes yetapa (Vieillot, 1818)] & Streamer-tailed Tyrant & 1,4 & 10 \\
\hline Cnemotriccus fuscatus (Wied, 1831) & Fuscous Flycatcher & $2,3,4,5$ & $\mathrm{BD}$ \\
\hline Lathrotriccus euleri (Cabanis, 1868) & Euler's Flycatcher & $1,2,4,5$ & $\mathrm{BD}$ \\
\hline [Knipolegus lophotes Boie, 1828] & Crested Black-Tyrant & 4 & 10 \\
\hline Xolmis cinereus (Vieillot, 1816) & Gray Monjita & $1,4,5$ & $\mathrm{BD}$ \\
\hline [Xolmis velatus (Lichtenstein, 1823)] & White-rumped Monjita & 4 & 10 \\
\hline \multicolumn{4}{|l|}{ Vireonidae } \\
\hline Cyclarhis gujanensis (Gmelin, 1789) & Rufous-browed Peppershrike & $1,2,3,4,5$ & $A D$ \\
\hline [Pachysylvia muscicapina (Sclater \& Salvin, 1873)] & Buff-cheeked Greenlet & 1 & 10 \\
\hline Vireo olivaceus (Linnaeus, 1766) & Red-eyed Vireo & 5 & $A D$ \\
\hline Vireo chivi (Vieillot, 1817) & Chivi Vireo & $1,2,3,4,5$ & $A D$ \\
\hline \multicolumn{4}{|l|}{ Corvidae } \\
\hline Cyanocorax cyanomelas (Vieillot, 1818) & Purplish Jay & $1,2,3,4,5$ & $A D$ \\
\hline Cyanocorax cristatellus (Temminck, 1823) CE & Curl-crested Jay & $1,2,4,5$ & $\mathrm{BD}$ \\
\hline \multicolumn{4}{|l|}{ Hirundinidae } \\
\hline [Pygochelidon cyanoleuca (Vieillot, 1817)] & Blue-and-white Swallow & 4 & 10 \\
\hline Stelgidopteryx ruficollis (Vieillot, 1817) & Southern Rough-winged Swallow & $1,2,3,4,5$ & $\mathrm{BD}$ \\
\hline Progne tapera (Vieillot, 1817) & Brown-chested Martin & 4,5 & $\mathrm{BD}$ \\
\hline Progne chalybea (Gmelin, 1789) & Gray-breasted Martin & 4,5 & $\mathrm{BD}$ \\
\hline [Tachycineta leucorrhoa (Vieillot, 1817)] & White-rumped Swallow & 4 & 10 \\
\hline [Hirundo rustica Linnaeus, 1758] & Barn Swallow & 4 & 10 \\
\hline \multicolumn{4}{|l|}{ Troglodytidae } \\
\hline Troglodytes musculus Naumann, 1823 & Southern House Wren & $1,2,4,5$ & $\mathrm{BD}$ \\
\hline Campylorhynchus turdinus (Wied, 1831) & Thrush-like Wren & $1,2,3,4,5$ & $A D$ \\
\hline Pheugopedius genibarbis (Swainson, 1838) & Moustached Wren & $1,2,3,4,5$ & $A D$ \\
\hline [Cantorchilus leucotis (Lafresnaye, 1845)] & Buff-breasted Wren & 1,4 & 10 \\
\hline \multicolumn{4}{|l|}{ Donacobiidae } \\
\hline [Donacobius atricapilla (Linnaeus, 1766)] & Black-capped Donacobius & 4 & 10 \\
\hline \multicolumn{4}{|l|}{ Polioptilidae } \\
\hline [Polioptila dumicola (Vieillot, 1817)] & Masked Gnatcatcher & $1,4,5$ & 10 \\
\hline \multicolumn{4}{|l|}{ Turdidae } \\
\hline Catharus fuscescens (Stephens, 1817) & Veery & $2,4,5$ & $A D$ \\
\hline Turdus leucomelas Vieillot, 1818 & Pale-breasted Thrush & $1,2,3,4,5$ & $A D$ \\
\hline [Turdus fumigatus Lichtenstein, 1823] & Cocoa Thrush & 2,4 & 10 \\
\hline Turdus hauxwelli Lawrence, 1869 & Hauxwell's Thrush & 5 & $A D$ \\
\hline
\end{tabular}




\begin{tabular}{|c|c|c|c|}
\hline Taxon & English name & Source & Record type \\
\hline Turdus rufiventris Vieillot, 1818 & Rufous-bellied Thrush & $1,4,5$ & $\mathrm{BD}$ \\
\hline Turdus amaurochalinus Cabanis, 1850 & Creamy-bellied Thrush & $1,2,4,5$ & $A D$ \\
\hline [Turdus albicollis Vieillot, 1818] & White-necked Thrush & 1,4 & 10 \\
\hline \multicolumn{4}{|l|}{ Mimidae } \\
\hline Mimus saturninus (Lichtenstein, 1823) & Chalk-browed Mockingbird & $1,2,4,5$ & $A D$ \\
\hline \multicolumn{4}{|l|}{ Passerellidae } \\
\hline Zonotrichia capensis (Statius Müller, 1776) & Rufous-collared Sparrow & $2,4,5$ & $\mathrm{BD}$ \\
\hline Ammodramus humeralis (Bosc, 1792) & Grassland Sparrow & $1,2,4,5$ & $\mathrm{BD}$ \\
\hline Arremon taciturnus (Hermann, 1783) & Pectoral Sparrow & $1,2,4,5$ & $A D$ \\
\hline Arremon flavirostris Swainson, 1838 & Saffron-billed Sparrow & 4,5 & $\mathrm{BD}$ \\
\hline \multicolumn{4}{|l|}{ Parulidae } \\
\hline [Setophaga pitiayumi (Vieillot, 1817)] & Tropical Parula & $1,2,4$ & 10 \\
\hline [Geothlypis aequinoctialis (Gmelin, 1789)] & Masked Yellowthroat & $1,2,4,5$ & 10 \\
\hline Basileuterus culicivorus (Deppe, 1830) & Golden-crowned Warbler & $1,2,4,5$ & $\mathrm{BD}$ \\
\hline Myiothlypis flaveola Baird, 1865 & Flavescent Warbler & $1,2,4,5$ & $\mathrm{BD}$ \\
\hline [Myiothlypis leucophrys (Pelzeln, 1868)] CE & White-striped Warbler & 4 & 10 \\
\hline \multicolumn{4}{|l|}{ Icteridae } \\
\hline [Psarocolius decumanus (Pallas, 1769)] & Crested Oropendola & 2,4 & 10 \\
\hline [Procacicus solitarius (Vieillot, 1816)] & Solitary Black Cacique & 4 & 10 \\
\hline [Cacicus haemorrhous (Linnaeus, 1766)] & Red-rumped Cacique & 1,4 & 10 \\
\hline Cacicus cela (Linnaeus, 1758) & Yellow-rumped Cacique & $1,2,3,4,5$ & $\mathrm{BD}$ \\
\hline Icterus pyrrhopterus (Vieillot, 1819) & Variable Oriole & $1,2,4,5$ & $\mathrm{BD}$ \\
\hline Icterus croconotus (Wagler, 1829) & Orange-backed Troupial & $1,2,3,4,5$ & $\mathrm{BD}$ \\
\hline Gnorimopsar chopi (Vieillot, 1819) & Chopi Blackbird & $1,2,3,4,5$ & $A D$ \\
\hline [Molothrus oryzivorus (Gmelin, 1788)] & Giant Cowbird & $1,2,4$ & 10 \\
\hline Molothrus bonariensis (Gmelin, 1789) & Shiny Cowbird & $1,4,5$ & $\mathrm{BD}$ \\
\hline \multicolumn{4}{|l|}{ Thraupidae } \\
\hline [Porphyrospiza caerulescens (Wied, 1830)] CE, NT & Blue Finch & 2,4 & 10 \\
\hline Neothraupis fasciata (Lichtenstein, 1823) CE, NT & White-banded Tanager & $1,4,5$ & $A D$ \\
\hline Cissopis leverianus (Gmelin, 1788) & Magpie Tanager & $1,2,3,4,5$ & $A D$ \\
\hline Schistochlamys melanopis (Latham, 1790) & Black-faced Tanager & $1,2,3,4,5$ & $A D$ \\
\hline [Paroaria capitata (d'Orbigny \& Lafresnaye, 1837)] & Yellow-billed Cardinal & 1,4 & 10 \\
\hline Tangara mexicana (Linnaeus, 1766) & Turquoise Tanager & $1,2,4,5$ & $\mathrm{BD}$ \\
\hline [Tangara chilensis (Vigors, 1832)] & Paradise Tanager & 1,4 & 10 \\
\hline Tangara sayaca (Linnaeus, 1766) & Sayaca Tanager & $1,2,4,5$ & $\mathrm{BD}$ \\
\hline Tangara palmarum (Wied, 1821) & Palm Tanager & $1,2,4,5$ & $\mathrm{BD}$ \\
\hline [Tangara cyanicollis (d'Orbigny \& Lafresnaye, 1837)] & Blue-necked Tanager & 1,4 & 10 \\
\hline Tangara cayana (Linnaeus, 1766) & Burnished-buff Tanager & $1,2,4,5$ & $\mathrm{BD}$ \\
\hline [Conirostrum speciosum (Temminck, 1824)] & Chestnut-vented Conebill & 4 & 10 \\
\hline Sicalis citrina Pelzeln, 1870 & Stripe-tailed Yellow-Finch & 4,5 & $\mathrm{BD}$ \\
\hline [Sicalis flaveola (Linnaeus, 1766)] & Saffron Finch & 1,4 & 10 \\
\hline [Chlorophanes spiza (Linnaeus, 1758)] & Green Honeycreeper & 1,4 & 10 \\
\hline [Hemithraupis flavicollis (Vieillot, 1818)] & Yellow-backed Tanager & 1,4 & 10 \\
\hline Hemithraupis guira (Linnaeus, 1766) & Guira Tanager & $1,2,4,5$ & $\mathrm{BD}$ \\
\hline Volatinia jacarina (Linnaeus, 1766) & Blue-black Grassquit & $1,2,3,4,5$ & $A D$ \\
\hline Eucometis penicillata (Spix, 1825) & Gray-headed Tanager & $2,4,5$ & $\mathrm{BD}$ \\
\hline Coryphospingus cucullatus (Statius Müller, 1776) & Red-crested Finch & $2,3,4,5$ & $\mathrm{BD}$ \\
\hline [Lanio versicolor (d'Orbigny \& Lafresnaye, 1837)] & White-winged Shrike-Tanager & 1,4 & 10 \\
\hline Lanio luctuosus (d'Orbigny \& Lafresnaye, 1837) & White-shouldered Tanager & $2,4,5$ & $\mathrm{BD}$ \\
\hline Lanio cristatus (Linnaeus, 1766) & Flame-crested Tanager & 5 & $\mathrm{BD}$ \\
\hline Tachyphonus rufus (Boddaert, 1783) & White-lined Tanager & $1,2,3,4,5$ & $A D$ \\
\hline Ramphocelus carbo (Pallas, 1764) & Silver-beaked Tanager & $1,2,3,4,5$ & $A D$ \\
\hline [Charitospiza eucosma Oberholser, 1905] CE, NT & Coal-crested Finch & 4 & 10 \\
\hline Tersina viridis (Illiger, 1811) & Swallow Tanager & $1,2,4,5$ & $\mathrm{BD}$ \\
\hline Cyanerpes caeruleus (Linnaeus, 1758) & Purple Honeycreeper & 1,4 & $\mathrm{BD}$ \\
\hline Cyanerpes cyaneus (Linnaeus, 1766) & Red-legged Honeycreeper & $1,2,4,5$ & $\mathrm{BD}$ \\
\hline Dacnis cayana (Linnaeus, 1766) & Blue Dacnis & $1,2,4,5$ & $\mathrm{BD}$ \\
\hline [Dacnis lineata (Gmelin, 1789)] & Black-faced Dacnis & 1,4 & 10 \\
\hline [Coereba flaveola (Linnaeus, 1758)] & Bananaquit & 1,4 & 10 \\
\hline Tiaris fuliginosus (Wied, 1830) & Sooty Grassquit & 3,4 & $A D$ \\
\hline [Sporophila lineola (Linnaeus, 1758)] & Lined Seedeater & 4 & 10 \\
\hline Sporophila plumbea (Wied, 1830) & Plumbeous Seedeater & 4,5 & $\mathrm{BD}$ \\
\hline [Sporophila collaris (Boddaert, 1783)] & Rusty-collared Seedeater & 4 & 10 \\
\hline [Sporophila nigricollis (Vieillot, 1823)] & Yellow-bellied Seedeater & $1,4,5$ & 10 \\
\hline Sporophila caerulescens (Vieillot, 1823) & Double-collared Seedeater & $2,4,5$ & $\mathrm{BD}$ \\
\hline [Sporophila leucoptera (Vieillot, 1817)] & White-bellied Seedeater & $1,4,5$ & 10 \\
\hline [Sporophila angolensis (Linnaeus, 1766)] & Chestnut-bellied Seed-Finch & $1,2,3,4$ & 10 \\
\hline [Sporophila maximiliani (Cabanis, 1851)] VU, CR* & Great-billed Seed-Finch & 4 & 10 \\
\hline
\end{tabular}




\begin{tabular}{|c|c|c|c|}
\hline Taxon & English name & Source & Record type \\
\hline [Emberizoides herbicola (Vieillot, 1817)] & Wedge-tailed Grass-Finch & $1,2,4$ & 10 \\
\hline Saltatricula atricollis (Vieillot, 1817) CE & Black-throated Saltator & $1,2,3,4,5$ & $A D$ \\
\hline Saltator maximus (Statius Müller, 1776) & Buff-throated Saltator & $1,2,3,4,5$ & $\mathrm{BD}$ \\
\hline [Saltator coerulescens Vieillot, 1817] & Grayish Saltator & 4,5 & 10 \\
\hline Saltator similis d'Orbigny \& Lafresnaye, 1837 & Green-winged Saltator & 4,5 & $\mathrm{BD}$ \\
\hline Cypsnagra hirundinacea (Lesson, 1831) CE & White-rumped Tanager & $1,2,4,5$ & $A D$ \\
\hline \multicolumn{4}{|l|}{ Cardinalidae } \\
\hline Piranga flava (Vieillot, 1822) & Hepatic Tanager & $1,2,4,5$ & $\mathrm{BD}$ \\
\hline [Cyanoloxia brissonii (Lichtenstein, 1823)] & Ultramarine Grosbeak & $1,2,4$ & 10 \\
\hline \multicolumn{4}{|l|}{ Fringillidae } \\
\hline Euphonia chlorotica (Linnaeus, 1766) & Purple-throated Euphonia & $2,4,5$ & $\mathrm{BD}$ \\
\hline [Euphonia violacea (Linnaeus, 1758)] & Violaceous Euphonia & 1,4 & 10 \\
\hline Euphonia laniirostris d'Orbigny \& Lafresnaye, 1837 & Thick-billed Euphonia & 4,5 & $\mathrm{BD}$ \\
\hline [Euphonia rufiventris (Vieillot, 1819)] & Rufous-bellied Euphonia & 1,4 & 10 \\
\hline \multicolumn{4}{|l|}{ Estrildidae } \\
\hline [Estrilda astrild (Linnaeus, 1758)] & Common Waxbill & $\mathrm{mp}$ & 10 \\
\hline
\end{tabular}

colonization of the sampled areas. Less represented at EESA, but still present, are species typical of Chaco and Pantanal wetland, such as Celeus lugubris (Malherbe, 1851) and Ortalis canicollis (Wagler, 1830), in addition to Phaethornis subochraceus Todd, 1915, recorded by Valadão (2012), a species closely associated to Chiquitanos dry forests (Vasconcelos and Hoffmann 2006).

We call attention to the elevational gradient of EESA. Our field survey evidenced the influence of altitudinal variation on the vegetation formations and consequently on the composition of the bird communities, which probably contributed to the high richness observed. According to Rosenzweig (1995), although the change in species composition along elevational gradients is a known fact, its relation to species' distribution patterns is not yet fully understood.

We found most Amazonian species at low elevations, from 220 to $400 \mathrm{~m}$. Other species, like Piranga flava (Vieillot, 1822), Hirundinea ferruginea (Gmelin, 1788), and several Cerrado endemics, like Saltatricula atricollis, Cypsnagra hyrundinacea and Neothraupis fasciata, we recorded above $500 \mathrm{~m}$ in open cerrado. We observed a possible altitudinal replacement in some taxa, related to the variation of vegetation at different altitudes. It is the case with Formicivora rufa and F. grisea, also observed by Willis and Oniki (1990), and with Nystalus maculatus

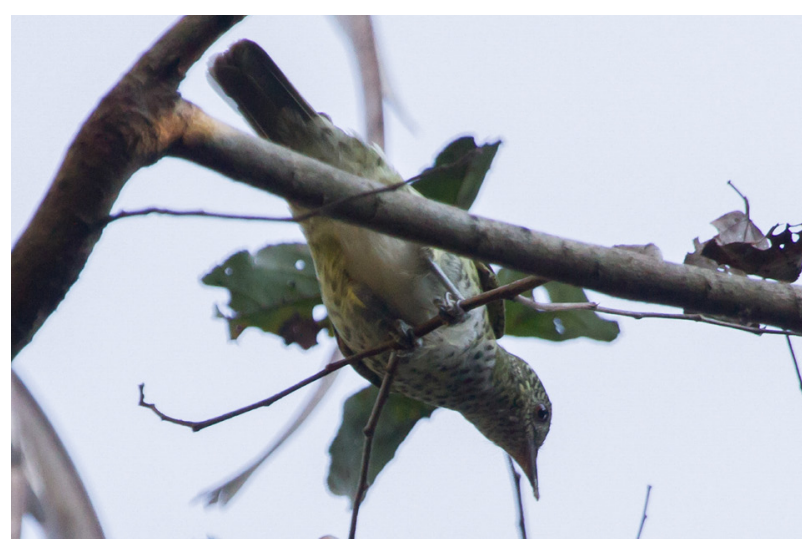

Figure 6. Record for Oxyruncus cristatus at Estação Ecológica da Serra das Araras. First documented record of the species for the state of Mato Grosso, Brazil. (Photo: BDV).

(Gmelin, 1788) and N. chacuru (Vieillot, 1816). In both cases, the second species of the pair were recorded in higher elevations and more open cerrado in EESA. We suggest thorough evaluations regarding the influence of the altitudinal gradient and the use and selection of habitats by birds in the EESA.

In our survey, conducted about 35 years after the creation of EESA, we noted the absence and/or low density, in area A, of some taxa mentioned by Silva and Oniki (1988) and Willis and Oniki (1990). Species associated

Table 4. Tertiary list including birds recorded at Estação Ecológica da Serra das Araras with no documented evidence, imprecise identification or locality, and of improbable occurrence in the area.

\footnotetext{
Netta peposaca (Vieillot, 1816): Species listed by Valadão (2012). No context given. A southern migrant, probably a vagrant at EESA.

Chaetura egregia Todd, 1916: Species listed by Willis and Oniki (1990) as of dubious identification.

Chaetura chapmani Hellmayr, 1907: Species listed by Willis and Oniki (1990) as of dubious identification.

Notharchus macrorhynchos (Gmelin, 1788): Listed by Valadão (2012). Record outside taxon's known geographical range. Probable nomenclatural confusion. Notharchus macrorhynchos was listed by Silva and Oniki (1988), before the taxon was split; this record is included in our primary list as N. hyperrhynchus.

Epinecrophylla haematonota (Sclater, 1857): Listed by Valadão (2012). Record outside taxon's known geographical range. Even considering the taxonomic revision of the haematonota complex, no taxon of this group is likely to occur at EESA, see Whitney et al. (2013).

Thamnophilus punctatus (Shaw, 1809): Listed by Valadão (2012). Record outside taxon's known geographical range. Probable nomenclatural confusion. The records of T. punctatus presented by Silva and Oniki (1988), Willis and Oniki (1990) and Oniki and Oliveira (2002) actually refer to Thamnophilus pelzelni Hellmayr, 1924, see Isler et al. (1997) and Piacentini et al. (2015).

Schiffornis sp. Bonaparte, 1854: Listed by Silva and Oniki (1988) only at genus level.

Icterus hauxwelli: Listed by Willis and Oniki (1990). Taxon not considered valid. Currently treated as a variation of Icterus croconotus (Wagler 1829), see Mallet-Rodrigues (2008), Lopes et al. (2009) and Piacentini et al. (2015).
} 
with altered areas, like Gnorimopsar chopi and Furnarius rufus, do not occur in this area today, due to the environmental changes brought about by the creation of the protected area. According to Pinheiro and Durigan (2009), anthropic pressure precludes some campo and savanna vegetation from reaching the edaphoclimatic climax of highest phytomass. Cessation of agricultural activities, as well as protection against wild fires, enable a gradual evolution from open formations to denser ones, resulting in a considerable increase in carbon stock.

However, some species typical of cerrado lost their habitats with the increasing density of the vegetation in some areas of EESA, where forest species were favored. Similar results were observed in state of São Paulo by Willis (2006). For example, S. atricollis, C. hirundinacea (Silva and Oniki 1988, Willis and Oniki 1990, Valadão 2012) and N. fasciata (Silva and Oniki 1988, Valadão 2012), previously recorded in area A, were not detected there in the course of our inventory.

We also highlight the presence of birds typical of rural areas (Vitorino et al. 2018), observed in the southernmost part of the EESA, due to territorial overlapping with a private property. Expansion of agriculture in this region may compromise important habitats, like the areas of open cerrado above $700 \mathrm{~m}$ and environments with rock outcrops present in this region.

As for species previously recorded at EESA but absent from our survey, we highlight Columbina cyanopis, included in the secondary list. In all, documented records for this species are extremely rare, which underscores the importance of the reports at EESA even if undocumented. Only 8 preserved specimens are known: 5 specimens were collected in the Cuiabá region, state of Mato Grosso, from 1823 to 1825; another was collected in state of São Paulo in 1904 and 2 others in state of Goiás in 1940-1941 (Baptista et al. 2018). In 2015, a new population was discovered in state of Minas Gerais (Bessa 2016 oral presentation). Our sampling effort during the expeditions at EESA in an attempt to record this species is not sufficient to infer that it does not occur in the area. We also point out that during our searches, we still did not know the voice of the species, which was released in early 2018. We thus encourage new expeditions aimed at detecting and documenting its presence at EESA.

We also call attention to the high number of species included in the secondary list. Until our inventory, documenting the species found in EESA was not a priority, which compromises somewhat the robustness of our results. We suggest that the next studies carried out in the EESA give special attention to species documentation, for future updates of the consolidated list, using the same criteria adopted herein. We underscore that, from a biogeographical standpoint, EESA is situated in a very interesting area, since it constitutes the distributional limit for several Amazonian species and some taxa of south-central Brazil.

In regard to significant documentation obtained by us at EESA, we mention the rare species Oxyruncus cris- tatus, previously recorded by Willis and Oniki (1990) and Valadão (2012). According to Brooke (2018), this species has an extremely disjunct distribution, occurring in parts of Colombia, Ecuador, Peru, Bolivia and Brazil. Gaps in the knowledge of its ecology and systematics persist, stemming from the difficulty of observing it in the field. This species is considered data deficient in Brazil (Pacheco at al 2007). Considering the numerous records for $O$. cristatus at EESA, we consider that the region has great potential as a site for ecological and taxonomic studies of this species.

In short, we state that EESA harbors a bird community typical of an ecotone area, especially with regard to Amazonian and Cerrado species. The species that make up this community are distributed among the various protected habitats within the EESA. A noteworthy number of Threatened and Near Threatened species are present, which depend both on forest and savanna formations, in addition to several endemic and rare species. The information presented in this paper highlights the importance of EESA for the conservation of birds in southwest of the state of Mato Grosso. In this way, we stress the importance of expanding EESA, to ensure, in addition to a more efficient protection, also the inclusion of other habitats present in the Província Serrana.

\section{Acknowledgements}

We wish to thank the Environmental Sciences graduate program at UNEMAT, Cáceres campus; Coordenação de Aperfeiçoamento de Pessoal de Nível Superior for MSc scholarships granted to BDV and AVBF; Marcelo Andrade from the Instituto Chico Mendes de Conservação da Biodiversidade and team, for his support throughout the project; Vitor Piacentini, Luciano Faria, Marcelo Vasconcelos, João Pinho and Wilkinson Lázaro for valuable discussions about the work; LF, VP and Sidnei Dantas for helping to determine some species; the UNEMAT biology department undergraduates who accompanied us in some field expeditions; the agencies that provided us with licenses, especially SISBio/IBAMA for collection permits and CEMAVE for supplying us with bird bands; and the collection curators for their prompt assistance, especially at UFMT and MPEG.

\section{Authors' Contributions}

BDV: project conception, in situ data collection, species identification, compilation and review of occurrence records, and writing of the manuscript. AVBF: project conception, in situ data collection, species identification, and review of the manuscript. SKI and JRSN: review of the manuscript.

\section{References}

Aleixo A, Guilherme E (2010) Avifauna da Estação Ecológica do Rio Acre, estado do Acre, na fronteira Brasil/Peru: composição, distribuição ecológica e registros relevantes. Boletim do Museu Para- 
ense Emilio Goeldi, Ciências Naturais 5 (3): 279-309.

Baptista LF, Trail PW, Horblit HM, Boesman P, Sharpe CJ (2018) Blue-eyed Ground-dove (Columbina cyanopis). In: del Hoyo J, Elliott A, Sargatal J, Christie DA, de Juana E (Eds) Handbook of the Birds of the World Alive. Lynx Edicions, Barcelona. https://www.hbw. com/node/54218. Accessed on 2018-03-04.

Bessa R (2016) Oral presentation. $11^{\circ}$ Encontro Brasileiro de Observação de Aves, São Paulo, Brazil.

Bibby CJ, Jones M, Marsden S (2000) Bird Surveys (Expedition Field Techniques Series). BirdLife International, Cambridge, 134 pp.

BirdLife International and Handbook of the Birds of the World (2016) Bird Species Distribution Maps of the World. Version 6.0.

Brazil (1994) Manual de Anilhamento de Aves Silvestres. 2nd ed. revista e ampliada. Instituto Brasileiro do Meio Ambiente e dos Recursos Naturais Renováveis, Brasília, 148pp.

Brazil (2016a) Plano de Manejo Estação Ecológica da Serra das Araras. Ministério do Meio Ambiente. Brasília, 252 pp.

Brazil (2016b) Sumário Executivo do Livro vermelho da fauna brasileira ameaçada de extinção. Instituto Chico Mendes de Conservação da Biodiversidade (ICMBio). Brazil: Ministério do Meio Ambiente (MMA), 75 pp.

Brooke M (2018) Sharpbill (Oxyruncus cristatus). In: del Hoyo J, Elliott A, Sargatal J, Christie DA, de Juana E (Eds) Handbook of the Birds of the World Alive. Lynx Edicions, Barcelona. https:// www.hbw.com/node/56991. Accessed on 2018-01-16.

Carlos CJ, Straube FC, Pacheco JF (2010) Conceitos e definições sobre documentação de registros ornitológicos e critérios para a elaboração de listas de aves para os estados brasileiros. Revista Brasileira de Ornitologia 18 (4): 355-361.

Cavalcanti RB (1999) Bird species richness and conservation in the Cerrado Region of Central Brazil. Studies in Avian Biology 19: 244-249.

Cavarzere V, Moraes GP, Donatelli RJ (2009) Avifauna da Estação Ecológica dos Caetetus, interior de São Paulo, Brasil. Papéis Avulsos de Zoologia 49 (35): 477-485. http://doi.org/10.1590/S003110492009003500001

D’Angelo Neto N, Venturin N, Oliveira-Filho, AT, Costa FAF (1998) Avifauna de quatro fisionomias florestais de pequeno tamanho (5-8 ha) no campus da UFLA. Revista Brasileira de Biologia 58 (3): 463-472. http://doi.org/10.1590/S0034-71081998000300011

del Hoyo J, Elliott A, Sargatal J, Christie DA, De Juana E (2017) Handbook of the Birds of the World Alive. Lynx Edicions, Barcelona.

Hassler ML (2005) A importância das Unidades de Conservação no Brasil. Sociedade \& Natureza 17 (33): 79-89.

Herzog SK, Kessler ME, Cahill TM (2002) Estimating species richness of tropical bird communities from Rapid Assessment Data. The Auk 119: 749-769. http://doi.org/bw2xj3

Isler ML, Isler PR, Whitney BM (1997) Biogeography and systematics of the Thamnophilus punctatus (Thamnophilidae) complex. Ornithological Monographs 48: 355-381.

IUCN (2017) The IUCN Red List of Threatened Species. International Union for Conservation of Nature and Natural Resources (IUCN). Version 2017-2. www.iucnredlist.org. Accessed on 2017-10-10.

Karr JR (1981) Surveying birds with mist nets. Studies in Avian Biology 6: 62-67.

Keyes BE, Grue CE (1982) Capturing birds with mist nets: a review. North American Bird Bander 7 (1): 2-14.

Kottek M, Grieser J, Beck C, Rudolf B, Rubel F (2006) World map of the Köppen-Geiger climate classification updated. Meteorologische Zeitschrift 15 (3): 259-263. https://doi.org/10.1127/09412948/2006/0130

Lopes LE, Pinho JB, Bernardon B, Oliveira FF, Bernardon G, Ferreira LP, Vasconcelos MF, Maldonado-Coelho M, Nóbrega PFA, Rubio TC (2009) Aves da Chapada dos Guimarães, Mato Grosso, Brasil: uma síntese histórica do conhecimento. Papéis Avulsos de Zoologia 49 (2): 9-47. http://doi.org/10.1590/S0031-10492009000200001

Lopes LE, Pinho JB, Ortiz A, Evangelista MM, Silveira LF, Schunck F, Develey PF (2016) Birds from Cáceres, Mato Grosso: the highest species richness ever recorded in a Brazilian non-forest region.
Revista Brasileira de Ornitologia 24 (2): 137-167.

MacKinnon J (1991) Field Guide to the Birds of Java and Bali. Gadjah Mada University Press, $391 \mathrm{pp}$.

Mallet-Rodrigues F (2008) Táxons de aves de validade questionável com ocorrência no Brasil. X-Oscines. Atualidades Ornitológicas 143: 8-9.

Motta-Junior JC, Granzinolli MAM, Develey, PF (2008) Aves da Estação Ecológica de Itirapina, estado de São Paulo, Brasil. Biota Neotropica 8 (3): 207-227. http://doi.org/cftrz2

Oniki Y, Oliveira DM (2002) Análise ecológica das aves capturadas na Estação Ecológica Serra das Araras, Mato Grosso, Brasil. Garcia de Orta, Série Zoologia 24: 1-6.

Oniki Y, Willis EO (1999) Body mass, cloacal temperature, morphometrics, breeding and molt of birds of the Serra das Araras region, Mato Grosso, Brazil. Ararajuba 7 (1):17-21.

Pacheco JF, Kirwan GM, Aleixo A, Whitney BM, Whittaker A, Minns J, Zimmer KJ, Fonseca PSM, Lima MFC, Oren DC (2007) An avifaunal inventory of the CVRD Serra dos Carajás project, Pará, Brazil. Cotinga 27: 15-30.

Payne R (2018) Pearly-breasted Cuckoo (Coccyzus euleri). In: del Hoyo J, Elliott A, Sargatal J, Christie DA, de Juana E (Eds) Handbook of the Birds of the World Alive. Lynx Edicions, Barcelona. https:// www.hbw.com/node/54892. Accessed on 2018-01-16.

Piacentini VQ, Aleixo A, Agne CE, Maurício GN, Pacheco JF, Bravo GA, Brito GRR, Naka LN, Olmos F, Posso S, Silveira LF, Betini GS, Carrano E, Franz I, Lees AC, Lima LM, Pioli D, Schunck F, Amaral FR, Bencke GA, Cohn-Haft M, Figueiredo LFA, Straube FC, Cesari E (2015) Annotated checklist of the birds of Brazil by the Brazilian Ornithological Records Committee / Lista comentada das aves do Brasil pelo Comitê Brasileiro de Registros Ornitológicos. Revista Brasileira de Ornitologia 23 (2): 91-298.

Pinheiro ES, Durigan G (2009) Dinâmica espaço-temporal (1962-2006) das fitofisionomias em unidade de conservação do Cerrado no sudeste do Brasil. Revista Brasileira de Botânica 32(3) 441-454.

Pinheiro RT, Dornas T (2009) Distribuição e conservação das aves na região do Cantão, Tocantins: ecótono Amazônia/Cerrado. Biota Neotropica 9 (1): 187-205.

Roos AL (2010) Capturando aves. In: Matter SV, Straube FC, Accordi I, Piacentini V, Cândido Jr JF. Ornitologia e conservação: ciência aplicada, técnicas de pesquisa e levantamento. Technical Books, Rio de Janeiro, 295-312.

Rosenzweig ML (1995) Species Diversity in Space and Time. Cambridge University Press, Cambridge, $436 \mathrm{pp}$.

Save Brasil (2010) Relatório anual 2010. http://www.savebrasil.org. br/wp-content/uploads/2013/11/Relatorio-Anual-de-Atividades_ SAVE-Brasil_2010.pdf. Accessed on 2018-02-27.

Save Brasil (2011) Relatório anual 2011. http://www.savebrasil.org. br/wp-content/uploads/2013/11/Relatorio-Anual-de-Atividades SAVE-Brasil_2011.pdf. Accessed on 2018-02-27.

Sick H (1997) Ornitologia brasileira. 2nd ed. Nova Fronteira, Rio de Janeiro, Brazil, 912 pp.

Silva JM, Oniki Y (1988) Lista preliminar da Avifauna da Estação Ecológica Serra das Araras, Mato Grosso, Brasil. Boletim do Museu Paraense Emilio Goeldi 4 (2): 123-143.

Silva JMC (1995) Birds of the Cerrado region, South America. Steenstrupia 21: 69-92.

Silva JMC, Bates JM (2002) Biogeographic patterns and conservation in the South American Cerrado: a tropical savanna hotspot. Bioscience 52 (3) 225-234.

Silveira LF, D’Horta FM (2002) A Avifauna da região de Vila Bela da Santíssima Trindade, Mato Grosso. Papéis Avulsos de Zoologia 42 (10) 265-286. http://doi.org/10.1590/S0031-10492002001000001

Somenzari M, Silveira LF, Piacentini VQ, Rego MA, Schunk F, Cavarzere V (2011) Birds of an Amazonia-Cerrado ecotone in Southern Pará, Brazil, and the efficiency of associating multiple methods in avifaunal inventories. Revista Brasileira de Ornitologia 19 (2): $260-275$.

Straube FC, Bianconi GV (2002) Sobre a grandeza e a unidade utilizada 
para estimar esforço de captura com utilização de redes-de-neblina. Chiroptera Neotropical 8 (1-2): 150-152.

Valadão RM (2012) As aves da Estação Ecológica Serra das Araras, Mato Grosso, Brasil. Biota Neotropica 12 (3): 263-281.

Vasconcelos MF, Hoffmann D (2006) Os Bosques Secos Chiquitanos também são nossos! Atualidades Ornitológicas 130: 10-11.

Vitorino BD, Rodrigues MB, Frota AVB, Avelar DM, Rodrigues WL, Ikeda Castrillon SK, Nunes JRS (2018) Birds of rural landscape in the Midwest region of the state of Minas Gerais, Brazil. Neotropical Biology and Conservation 13 (1): 62-73 http:// doi.org/10.4013/ nbc.2018.131.08

Whitney BM, Isler ML, Bravo GA, Aristizábal N, Schunck F, Silveira LF, Piacentini VQ (2013) A new species of Epinecrophylla antwren from the Aripuanã-Machado interfluvium in central Amazonian Brazil with revision of the "stipple-throated antwren" complex. In: del Hoyo J, Elliott A, Sargatal J, Christie D (Eds) Handbook of the Birds of the World. Special volume: new species and global index. Lynx Edicions, Barcelona, 263-267.

Willis EO (2006) Protected cerrado fragments grow up and lose even metapopulational birds in Central São Paulo, Brazil. Brazilian
Journal of Biology 66 (3): 829-837. http://doi.org/10.1590/S151969842006000500008

Willis EO, Oniki Y (1990) Levantamento preliminar das aves de inverno em dez áreas do sudoeste de Mato Grosso, Brasil. Ararajuba 1: 19-38.

Winkler H, Christie DA, Kirwan GM, Sharpe CJ (2018) Ringed Woodpecker (Celeus torquatus). In: del Hoyo J, Elliott A, Sargatal J, Christie DA, de Juana E (Eds) Handbook of the Birds of the World Alive. Lynx Edicions, Barcelona. https://www.hbw.com/ node/55418. Accessed on 2018-01-16.

Züchner T, Kirwan GM, Boesman P (2018a) Dot-eared Coquette (Lophornis gouldii). In: del Hoyo J, Elliott A, Sargatal J, Christie DA, de Juana E (Eds) Handbook of the Birds of the World Alive. Lynx Edicions, Barcelona. https://www.hbw.com/node/55418. Accessed on 2018-01-16.

Züchner T, Kirwan GM, Boesman P (2018b) Frilled Coquette (Lophornis magnificus). In: del Hoyo J, Elliott A, Sargatal J, Christie DA, de Juana E (Eds) Handbook of the Birds of the World Alive. Lynx Edicions, Barcelona. https://www.hbw.com/node/55420. Accessed on 2018-01-16.

\section{Appendix}

Table A1. List of bird specimens collected at Estação Ecológica da Serra das Araras before the present study and deposited in scientific collections.

\begin{tabular}{|c|c|c|c|}
\hline Taxon & English name & UFMT catalog no. & MPEG catalog no. \\
\hline \multicolumn{4}{|l|}{ Accipitriformes Bonaparte, 1831} \\
\hline \multicolumn{4}{|l|}{ Accipitridae Vigors, 1824} \\
\hline Rupornis magnirostris (Gmelin, 1788) & Roadside Hawk & & 37785 \\
\hline \multicolumn{4}{|l|}{ Gruiformes Bonaparte, 1854} \\
\hline \multicolumn{4}{|l|}{ Rallidae Rafinesque, 1815} \\
\hline Laterallus viridis (Statius Müller, 1776) & Russet-crowned Crake & 0033 & \\
\hline \multicolumn{4}{|l|}{ Columbiformes Latham, 1790} \\
\hline \multicolumn{4}{|l|}{ Columbidae Leach, 1820} \\
\hline Claravis pretiosa (Ferrari-Perez, 1886) & Blue Ground-Dove & 0089,0172 & \\
\hline Leptotila verreauxi Bonaparte, 1855 & White-tipped Dove & 0040 & \\
\hline \multicolumn{4}{|l|}{ Cuculiformes Wagler, 1830} \\
\hline \multicolumn{4}{|l|}{ Cuculidae Leach, 1820} \\
\hline Crotophaga ani Linnaeus, 1758 & Smooth-billed Ani & 0065 & \\
\hline Tapera naevia (Linnaeus, 1766) & Striped Cuckoo & 0073 & \\
\hline \multicolumn{4}{|l|}{ Caprimulgiformes Ridgway, 1881} \\
\hline \multicolumn{4}{|l|}{ Caprimulgidae Vigors, 1825} \\
\hline Hydropsalis torquata (Gmelin, 1789) & Scissor-tailed Nightjar & & 37771 \\
\hline \multicolumn{4}{|l|}{ Apodiformes Peters, 1940} \\
\hline \multicolumn{4}{|l|}{ Trochilidae Vigors, 1825} \\
\hline Glaucis hirsutus (Gmelin, 1788) & Rufous-breasted Hermit & 0903 & 37787 \\
\hline Phaethornis nattereri Berlepsch, 1887 & Cinnamon-throated Hermit & & 37772 \\
\hline Eupetomena macroura (Gmelin, 1788) & Swallow-tailed Hummingbird & 0071 & \\
\hline Colibri serrirostris (Vieillot, 1816) & White-vented Violetear & 0055 & \\
\hline Anthracothorax nigricollis (Vieillot, 1817) & Black-throated Mango & & 37793 \\
\hline Thalurania furcata (Gmelin, 1788) & Fork-tailed Woodnymph & 0059,0086 & 37791 \\
\hline \multicolumn{4}{|l|}{ Coraciiformes Forbes, 1844} \\
\hline \multicolumn{4}{|l|}{ Momotidae Gray, 1840} \\
\hline Momotus momota (Linnaeus, 1766) & Amazonian Motmot & 0034 & 37777 \\
\hline \multicolumn{4}{|l|}{ Galbuliformes Fürbringer, 1888} \\
\hline \multicolumn{4}{|l|}{ Galbulidae Vigors, 1825} \\
\hline Brachygalba lugubris (Swainson, 1838) & Brown Jacamar & & 37789 \\
\hline \multicolumn{4}{|l|}{ Bucconidae Horsfield, 1821} \\
\hline Nystalus chacuru (Vieillot, 1816) & White-eared Puffbird & 0051 & \\
\hline Monasa nigrifrons (Spix, 1824) & Black-fronted Nunbird & 0072 & \\
\hline \multicolumn{4}{|l|}{ Piciformes Meyer \& Wolf, 1810} \\
\hline \multicolumn{4}{|l|}{ Ramphastidae Vigors, 1825} \\
\hline Pteroglossus castanotis Gould, 1834 & Chestnut-eared Aracari & & 37786 \\
\hline
\end{tabular}




\begin{tabular}{|c|c|c|c|}
\hline Taxon & English name & UFMT catalog no. & MPEG catalog no. \\
\hline \multicolumn{4}{|l|}{ Picidae Leach, 1820} \\
\hline Melanerpes cruentatus (Boddaert, 1783) & Yellow-tufted Woodpecker & 0075,0088 & 37781 \\
\hline Veniliornis passerinus (Linnaeus, 1766) & Little Woodpecker & 0054 & \\
\hline \multicolumn{4}{|l|}{ Psittaciformes Wagler, 1830} \\
\hline \multicolumn{4}{|l|}{ Psittacidae Rafinesque, 1815} \\
\hline Brotogeris chiriri (Vieillot, 1818) & Yellow-chevroned Parakeet & & 37776 \\
\hline \multicolumn{4}{|l|}{ Passeriformes Linnaeus, 1758} \\
\hline \multicolumn{4}{|l|}{ Thamnophilidae Swainson, 1824} \\
\hline Myrmophylax atrothorax (Boddaert, 1783) & Black-throated Antbird & & 37782,37783 \\
\hline Formicivora rufa (Wied, 1831) & Rusty-backed Antwren & 0053 & \\
\hline Thamnophilus pelzelni Hellmayr, 1924 & Planalto Slaty-Antshrike & & 37788 \\
\hline Pyriglena leuconota (Spix, 1824) & White-backed Fire-eye & & 37798 \\
\hline \multicolumn{4}{|l|}{ Dendrocolaptidae Gray, 1840} \\
\hline Sittasomus griseicapillus (Vieillot, 1818) & Olivaceous Woodcreeper & & 37773 \\
\hline Xiphorhynchus guttatoides (Lafresnaye, 1850) & Lafresnaye's Woodcreeper & 0067 & 37784 \\
\hline \multicolumn{4}{|l|}{ Furnariidae Gray, 1840} \\
\hline Furnarius rufus (Gmelin, 1788) & Rufous Hornero & & 37796 \\
\hline Synallaxis albescens Temminck, 1823 & Pale-breasted Spinetail & & 37775 \\
\hline \multicolumn{4}{|l|}{ Pipridae Rafinesque, 1815} \\
\hline Neopelma pallescens (Lafresnaye, 1853) & Pale-bellied Tyrant-Manakin & & 37797 \\
\hline Pipra fasciicauda Hellmayr, 1906 & Band-tailed Manakin & 0035,0077 & 37794,37795 \\
\hline Manacus manacus (Linnaeus, 1766) & White-bearded Manakin & $0049,0069,0094$ & \\
\hline Machaeropterus pyrocephalus (Sclater, 1852) & Fiery-capped Manakin & 0074,0098 & \\
\hline \multicolumn{4}{|l|}{ Tityridae Gray, 1840} \\
\hline Tityra semifasciata (Spix, 1825) & Masked Tityra & 0888 & \\
\hline \multicolumn{4}{|l|}{ Rhynchocyclidae Berlepsch, 1907} \\
\hline Poecilotriccus latirostris (Pelzeln, 1868) & Rusty-fronted Tody-Flycatcher & 0090 & \\
\hline Hemitriccus striaticollis (Lafresnaye, 1853) & Stripe-necked Tody-Tyrant & 0036 & \\
\hline \multicolumn{4}{|l|}{ Tyrannidae Vigors, 1825} \\
\hline Elaenia chilensis Hellmayr, 1927 & Chilean Elaenia & 0080,0081 & \\
\hline Elaenia chiriquensis Lawrence, 1865 & Lesser Elaenia & 0079 & \\
\hline Myiopagis gaimardii (d'Orbigny, 1839) & Forest Elaenia & 0096 & \\
\hline Phaeomyias murina (Spix, 1825) & Mouse-colored Tyrannulet & 0082 & \\
\hline Attila bolivianus Lafresnaye, 1848 & Dull-capped Attila & 0038 & \\
\hline Myiarchus tyrannulus (Statius Müller, 1776) & Brown-crested Flycatcher & 0048 & \\
\hline \multicolumn{4}{|l|}{ Vireonidae Swainson, 1837} \\
\hline Cyclarhis gujanensis (Gmelin, 1789) & Rufous-browed Peppershrike & 0037,0045 & \\
\hline Vireo chivi (Vieillot, 1817) & Chivi Vireo & 0091,0099 & \\
\hline \multicolumn{4}{|l|}{ Corvidae Leach, 1820} \\
\hline Cyanocorax cyanomelas (Vieillot, 1818) & Purplish Jay & 0083 & 37778 \\
\hline \multicolumn{4}{|l|}{ Troglodytidae Swainson, 1831} \\
\hline Campylorhynchus turdinus (Wied, 1831) & Thrush-like Wren & 0043,0044 & \\
\hline Pheugopedius genibarbis (Swainson, 1838) & Moustached Wren & 0047,0097 & \\
\hline \multicolumn{4}{|l|}{ Turdidae Rafinesque, 1815} \\
\hline Turdus leucomelas Vieillot, 1818 & Pale-breasted Thrush & 0039,0062 & \\
\hline \multicolumn{4}{|l|}{ Mimidae Bonaparte, 1853} \\
\hline Mimus saturninus (Lichtenstein, 1823) & Chalk-browed Mockingbird & & 37792 \\
\hline \multicolumn{4}{|l|}{ Icteridae Vigors, 1825} \\
\hline Gnorimopsar chopi (Vieillot, 1819) & Chopi Blackbird & 0060,0061 & \\
\hline Thraupidae Cabanis, 1847 & & & \\
\hline Neothraupis fasciata (Lichtenstein, 1823) & White-banded Tanager & & 37779 \\
\hline Cissopis leverianus (Gmelin, 1788) & Magpie Tanager & $0041,0046,0068,0084$ & 37774 \\
\hline Schistochlamys melanopis (Latham, 1790) & Black-faced Tanager & $0058,0078,0085,0746$ & \\
\hline Volatinia jacarina (Linnaeus, 1766) & Blue-black Grassquit & 0050,0095 & \\
\hline Tachyphonus rufus (Boddaert, 1783) & White-lined Tanager & 0063,0066 & \\
\hline Ramphocelus carbo (Pallas, 1764) & Silver-beaked Tanager & 0057 & \\
\hline Tiaris fuliginosus (Wied, 1830) & Sooty Grassquit & 0064 & \\
\hline Saltatricula atricollis (Vieillot, 1817) & Black-throated Saltator & $0070,0076,0093$ & \\
\hline Cypsnagra hirundinacea (Lesson, 1831) & White-rumped Tanager & & 37780,37790 \\
\hline
\end{tabular}

UFMT = Universidade do Estado de Mato Grosso; MPEG = Museu Paraense Emílio Goeldi. 